Building a comprehensive dataset for the validation of daylight simulation software, using complex "real architecture" 



\title{
Building a comprehensive dataset for the validation of daylight simulation software, using complex "real architecture"
}

\author{
By
}

Jake Osborne

A thesis submitted to the School of Architecture, Victoria University of Wellington in fulfilment of the requirements for the degree of Master of Building Science

Victoria University of Wellington

September 2012 

This thesis was submitted in fulfilment of the requirements of the Master of Building Science degree at Victoria University of Wellington School of Architecture and Design in 2012.

\section{Author:}

Jake Osborne

School of Architecture and Design

Victoria University of Wellington

jzsosborne@gmail.com

$+64-21-153-4266$

\section{Research Supervisor:}

Michael Donn

Director Centre for Building Performance Research

School of Architecture and Design

Victoria University of Wellington

michael.donn@vuw.ac.nz

Victoria University

+64-4-463-6221 work

PO Box 600

Wellington +64-21-611-280 mobile

New Zealand

+64-4-463-6204 work fax

+64-21-611-594 mobile fax 

This research focused on building a comprehensive dataset for use in validation studies of daylight simulation software. The aim of the set is to add to existing validation data to better cover a wide range of complexities and weather conditions. This will allow for not only the validation of simulation software, but the comparison of multiple simulators in their general strengths and weaknesses as well as feasibility for early 'sketch' design stages and complete building simulations. The set can also aid in the creation of valid simulation parameter starting points for designers.

The research examined the current 'gold standard' validation dataset from the BRE-IDMP, and found that while it provides excellent validation opportunities for simulators that can support its detailed patch-based sky model; an equally high quality dataset is needed for simulators that support more simplified skies. This is essential as most of the weather data for annual daylighting simulations available to designers, such as the US-DOE's collection of TMY data, can only be used in mathematical sky models such as the Perez all-weather model. It is also essential that real world, complex light-path scenarios commonly found in buildings be addressed by validation in addition to the simple single room, single opening tests which are prevalent in the daylight simulation field.

A dataset suite is proposed, similar to the BESTEST suite for energy simulation, which covers basic analytical test cases for lighting simulators, simple office scenarios and a complex shaded classroom in a tropical climate. The dataset is valuable for the testing of daylight simulators which make use of the common CIE general or Perez all-weather skies. These datasets were used in a trial validation of Autodesk's 3ds Max Design and Radiance, which included significant sensitivity testing of the two empirical datasets included in the suite. This demonstrated the usefulness of each dataset, and any issues with their data. It also highlighted the key inputs of any simulation model where designers must take significant care. 

First and foremost l'd like to thank my supervisor, Michael Donn, for his constant guidance and patience throughout this process.

Thanks also to all those at Autodesk, the research funders, for their technical assistance and understanding. Jena Shore, Brenton Wyett, Pierre-Felix Breton, Azam Khan, Ramtin Attar, Alex Tessier, Ebenezer Hailemariam, Adam Arbree, John Kennedy, Ken Pimentel Shane Griffith, and Debra Pothier.

Everyone at the Université de La Réunion for their assistance in collecting data, Aurélie Lenoir, François Garde, and Shaan Cory.

Many thanks to the Lawrence Berkeley National Laboratory: Andy McNeil, Eleanor Lee, Kyle Konis and Dennis Dibartolomeo, for the use of their data.

Thanks to Greg Ward and the rest of the Radiance-Online community for their assistance with Radiance.

And of course many thanks my friends, family and fellow building science and architecture postgrads for your continued support and comradeship. 

I Preface

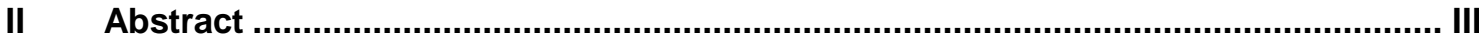

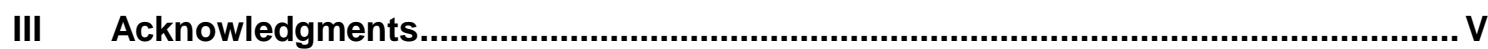

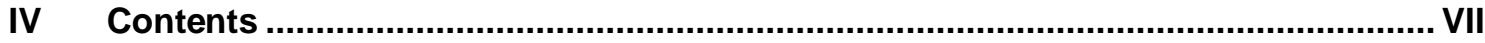

V List of Definitions \& Abbreviations ...................................................................... IX

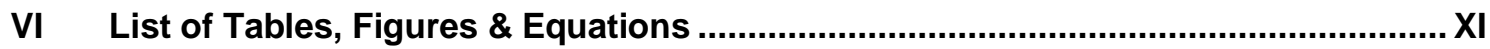

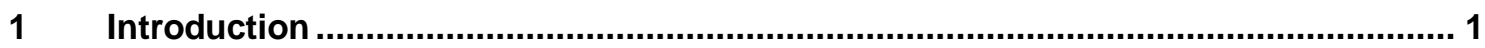

$1.1 \quad$ What is "complex" and why simulate it?

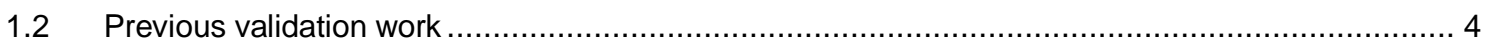

1.3 Why More Validation?

1.4 Aim

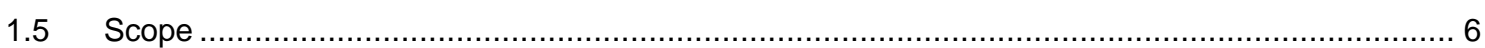

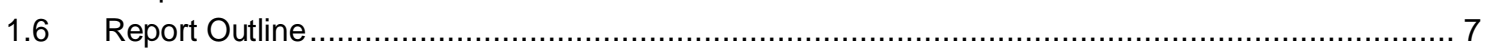

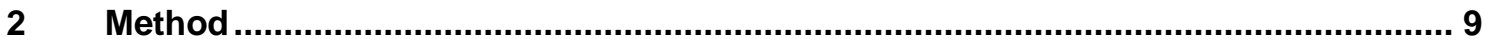

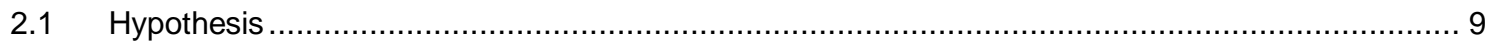

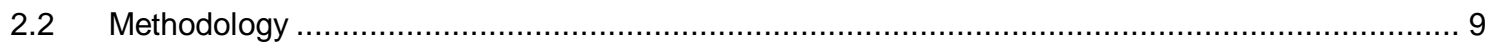

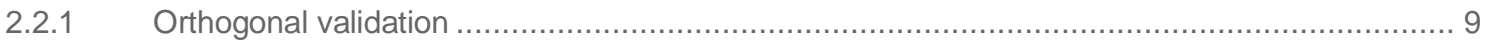

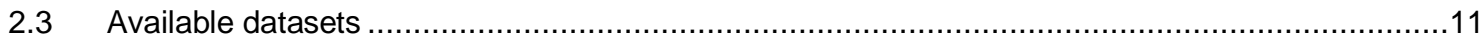

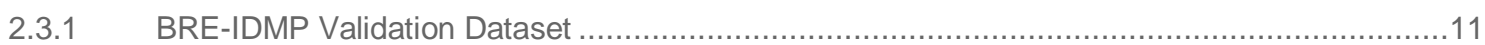

2.3.2 CIE 171:2006 - Test Cases to Assess the Accuracy of Lighting Computer Programs .............12

2.3.3 Lawrence Berkeley National Laboratory 71T test-bed facility .........................................12

2.3.4 Université de La Réunion Laboratoire de Physique du Bâtiment et des Systèmes ..................13

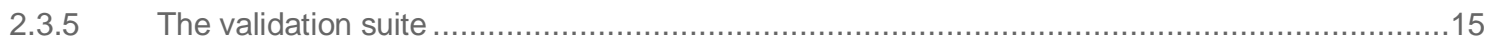

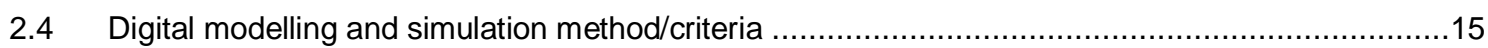

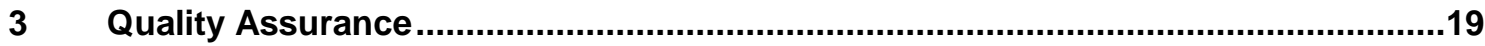

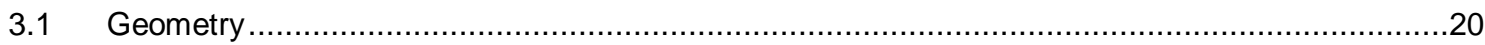

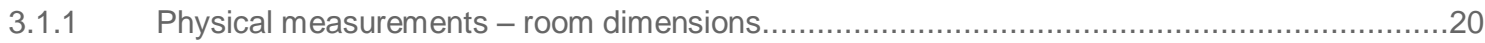

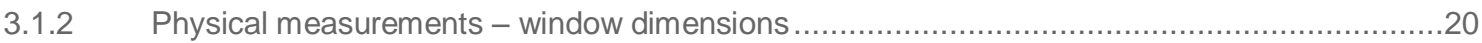

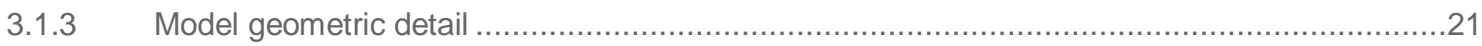

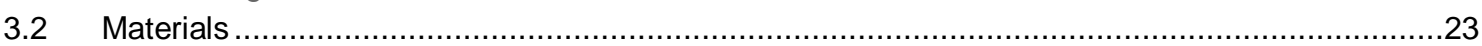

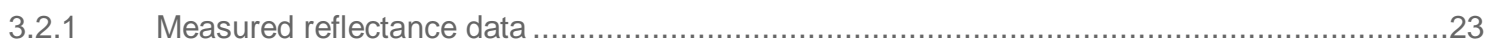

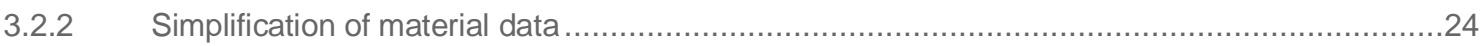

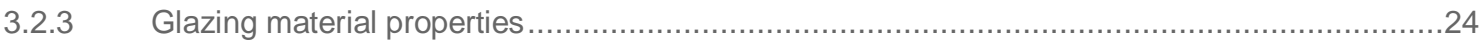

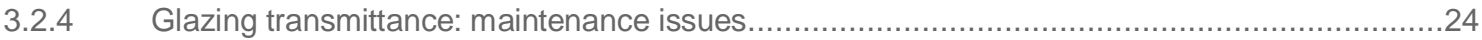

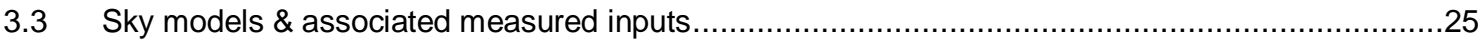

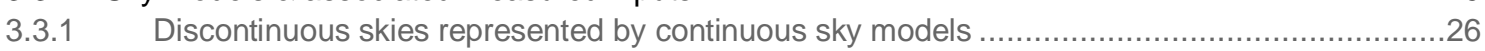

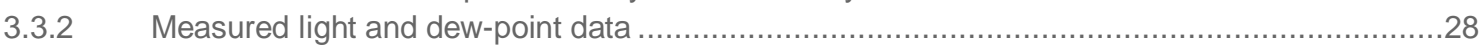

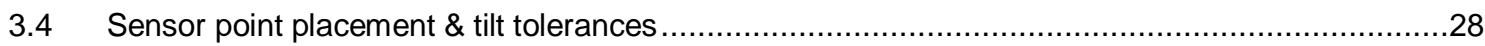

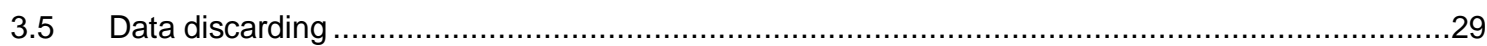

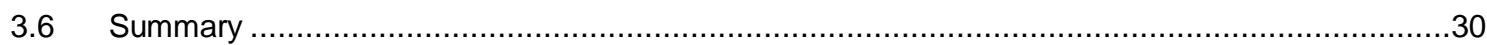

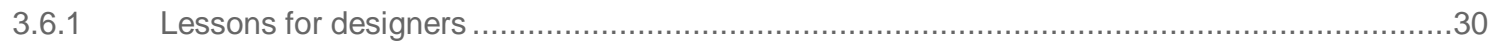

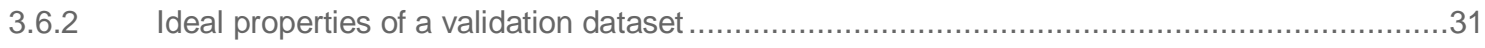

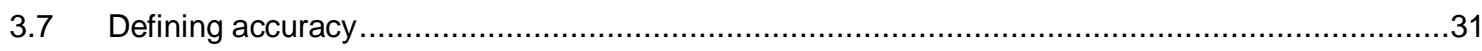

4 Validating 3ds Max Design and Radiance against the CIE 171:2006 test cases.......33

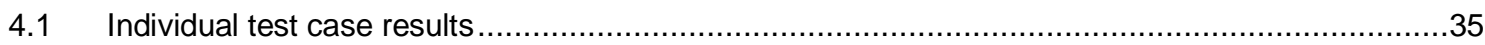

4.1.1 Test cases 4.1 - 4.3: Artificial lighting scenarios with diffuse inter-reflections .........................35

4.1.2 Test cases 4.4-4.6: Artificial lighting scenarios without inter-reflections..........................38

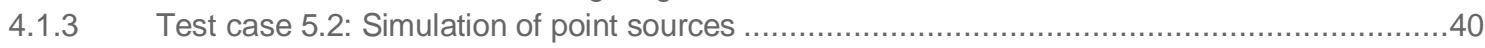




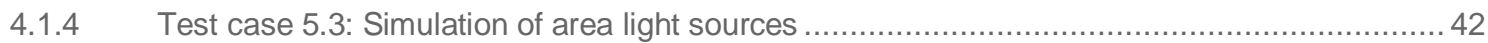

4.1.5 Test case 5.4: Luminous flux conservation ............................................................... 44

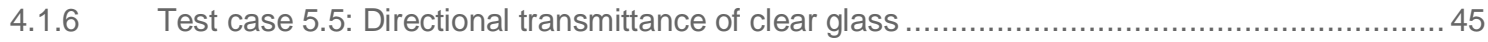

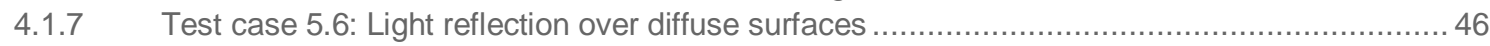

4.1.8 Test case 5.7: Diffuse reflection with internal obstructions ............................................49

4.1.9 Test case 5.8: Internal reflected component for diffuse surfaces ........................................5 50

4.1.10 Test cases 5.9 \& 5.10: Sky components for roof openings and CIE general sky types.............52

4.1.11 Test cases 5.11 \& 5.12: Sky + external reflected components for façade openings .................54

4.1.12 Test cases 5.13 \& 5.14: SC + ERC for obstructed façade openings................................56

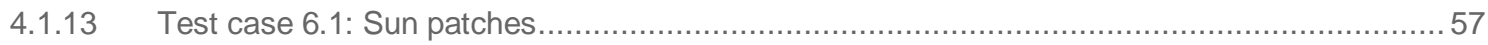

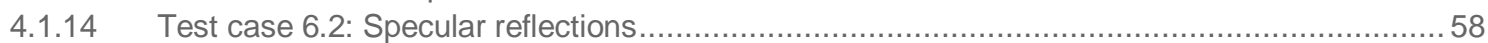

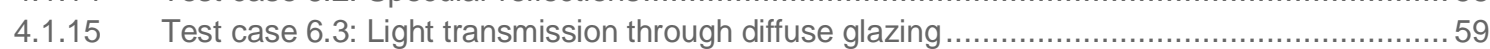

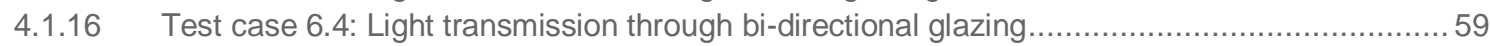

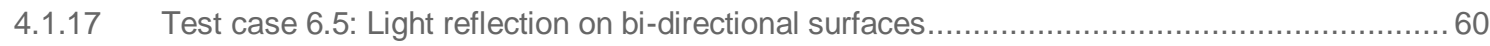

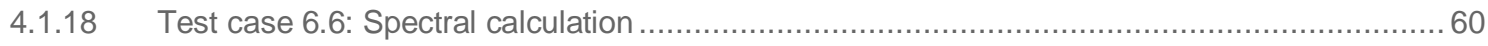

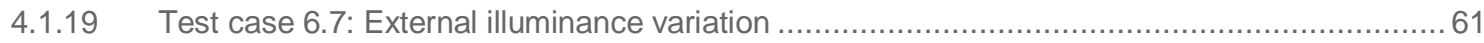

4.1.20 Test case 6.8: Daily and monthly variation of external illuminance ......................................... 61

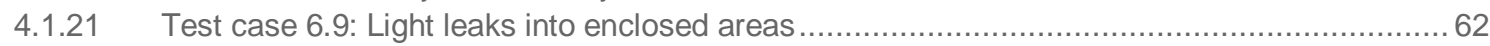

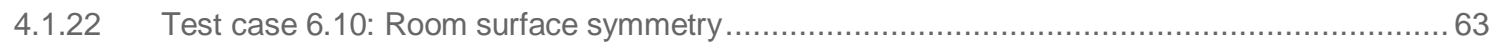

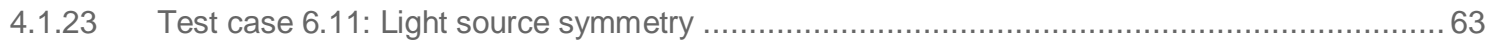

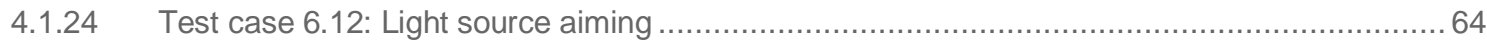

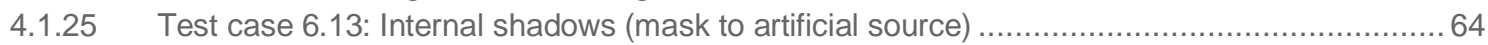

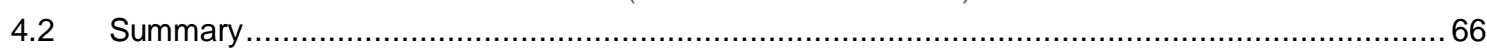

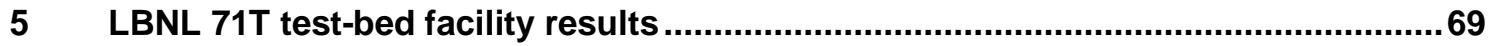

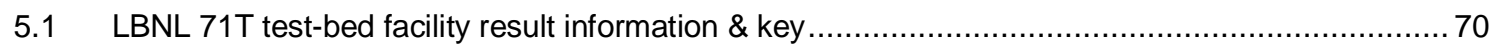

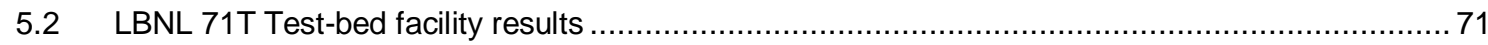

$6 \quad$ Université de La Réunion LPBS results............................................................... 77

6.1 Université de La Réunion LPBS result information \& key ............................................... 78

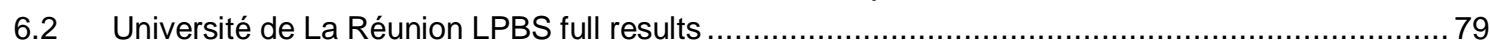

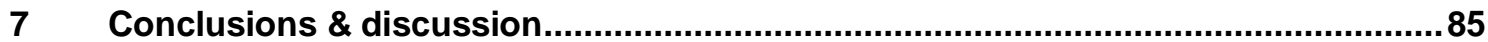

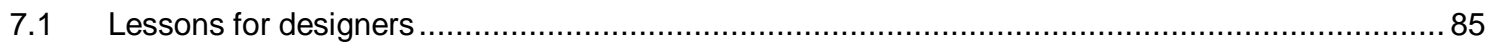

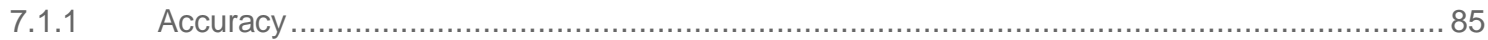

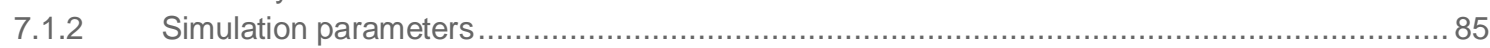

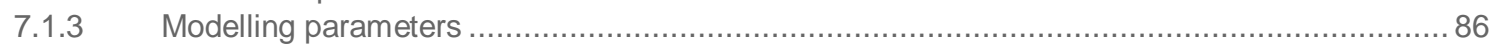

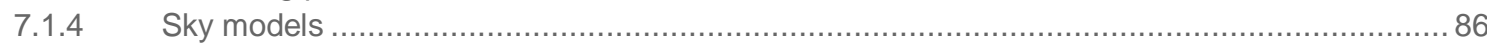

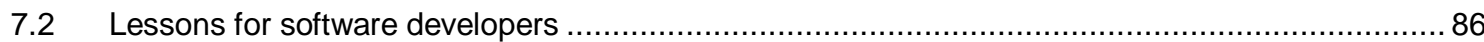

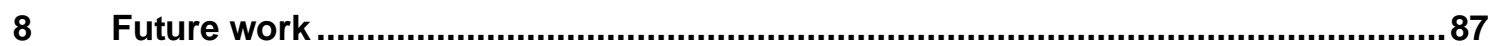

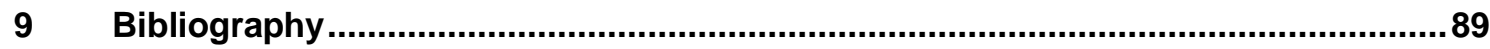

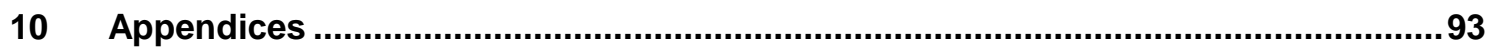

10.1 Appendix 1: 3ds Max Design \& Radiance simulation options \& parameters ...............................93

10.2 Appendix 3: LBNL 71T test-bed facility additional results ................................................ 95 


\section{Definitions}

Designers - Architects, engineers, lighting and daylighting consultants, green building practitioners, and anyone interested in producing buildings with quality daylighting and daylighting controls.

Final Gather - The backward raytracing engine of the mental ray light simulator within Autodesk 3ds Max Design.

Global Illumination - Can refer to two definitions; first the forward raytracing engine of the mental ray light simulator within Autodesk 3ds Max Design, as it will be used in this document. Second it can refer to the light in a digital model that is from secondary or diffuse sources, such as light that has been reflected from a matte painted wall or from the dome of the sky, rather than the sun itself. This document will instead refer to this as 'indirect' or 'diffuse' light to avoid confusion.

Turbidity - The amount of haziness in the sky, as opposed to cloudiness.

Dynamic Daylight Simulation - Daylight simulation that rather than being carried out for daylight factor or other metric for one or three times a year, instead covers many time-steps over the entirety of the year. An example of this is the Useful Daylight Index, which examines what portion of internal illuminances fall within a certain 'useful' range over an entire year.

Light-path - The path that light must travel from illumination source to measurement point or vice-versa in backward raytracing. Generally the shortest light-path in a simulation will be from the sun, directly through a pane of glass and on to a measurement point. A longer path may be through a pane of glass, off the top of a venetian blind blade, to the ceiling and on to a measurement point.

Test case - A given building design scenario associated with reference data, to be used for assessing a given aspect of a lighting simulation. (CIE TC $3.33,2005)$

Reference data - A set of values (calculated or measured) to be used as a reference when assessing the results of a simulation. (CIE TC 3.33, 2005)

Experimental/Empirical test case - A real-world case study where the reference data is based on experimental measurements inside a scale model or a test room. (CIE TC 3.33, 2005)

Analytical test case - A theoretical building design scenario wherein the reference data can be analytically calculated based on given assumptions (e.g. light source and surface photometry) and physical laws. (CIE TC 3.33, 2005)

\section{Abbreviations}

BRDF - Bi-directional Reflectance Distribution Function

BSDF - Bi-directional Scattering Distribution Function

FG - Final Gather

GI - Global Illumination

LBNL - Lawrence Berkeley National Laboratory

LPBS - Laboratoire de Physique du Bâtiment et des Systèmes (Laboratory of Building Physics and Systems)

MBE - Mean Bias Error

RGB (A) - Red Green Blue (Alpha)

TMY - Typical Meteorological Year

UDI - Useful Daylight Index 

Tables

Table 1: Dataset matrix 10

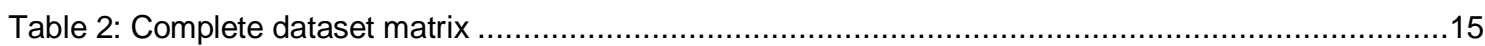

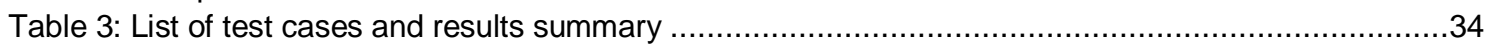

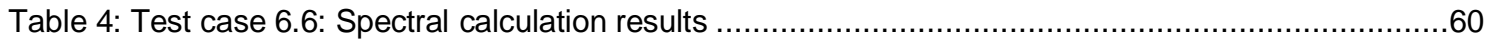

Table 5: LBNL 71T workplane Mean Bias Errors for 3ds Max Design ............................................73

Table 6: LBNL 71T workplane Mean Bias Errors for Radiance .......................................................73

Table 7: LPBS workplane Mean Bias Errors for 3ds Max Design..................................................81

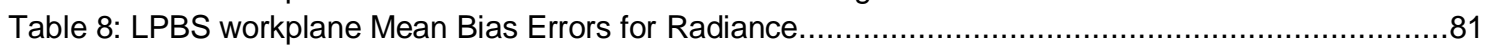

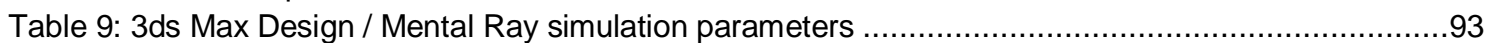

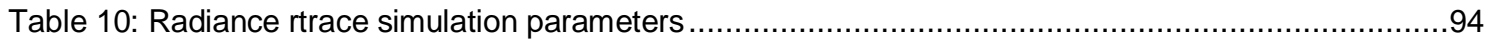

Table 11: LBNL 71T daily workplane Mean Bias Errors \& data points.........................................96

Figures

Figure 1: Examples of highly complex daylight systems (Ruck, et al., 2000) …................................. 3

Figure 2: Orthogonal validation procedure (Mardaljevic, 2002) ................................................... 9

Figure 3: LBNL 71T test-bed facility (photo courtesy Michael Donn) .................................................13

Figure 4: Université de La Réunion LPBS building (photo courtesy François Garde)............................14

Figure 5: LPBS office (left) and classroom (right) interiors (photos courtesy Shaan Cory) .....................14

Figure 6: Render of detailed CAD model of the LPBS building, without measured reflectances ...............21

Figure 7: LPBS model with original (left) and simplified (right) window and door geometry .......................22

Figure 8: LPBS model with original (left) and simplified shading devices (right) ................................22

Figure 9: LPBS model with fully simplified geometry and without measured reflectances ......................22

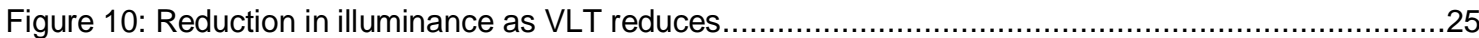

Figure 11: Sky-scanner luminance versus Perez sky model luminance examples (Mardaljevic, 2000) ......27

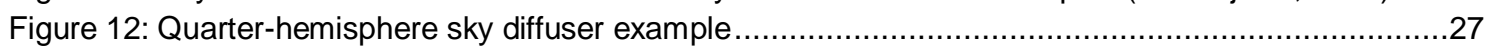

Figure 13: Typical shadow movements over measurement point.................................................29

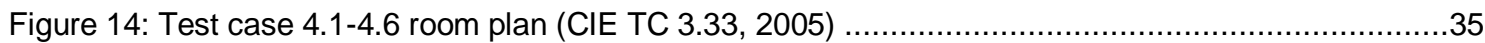

Figure 15: Test case 4.1 Artificial lighting scenario; CFL, grey wall initial results ..............................36

Figure 16: Test case 4.1: Artificial lighting scenario; CFL, grey wall results ........................................37

Figure 17: Test case 4.2: Artificial lighting scenario; Opal luminaire, grey wall results .............................37

Figure 18: Test case 4.3: Artificial lighting scenario; SSR, grey wall results ......................................38

Figure 19: Test case 4.4: Artificial lighting scenario; CFL, black wall results .....................................39

Figure 20: Test case 4.5: Artificial lighting scenario; Opal luminaire, black wall results ...........................39

Figure 21: Test case 4.6: Artificial lighting scenario; SSR, black wall results ...................................40

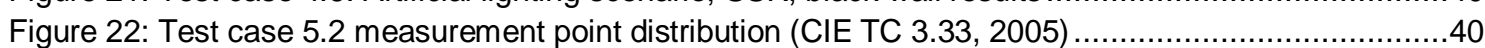

Figure 23: Test case 5.2: Simulation of uniform diffuse point sources results .....................................41

Figure 24: Test case 5.2: Simulation of photometric point sources results ......................................41

Figure 25: Test case 5.3 geometry and measurement points (CIE TC 3.33, 2005) ...........................42

Figure 26: Test case 5.3: Simulation of diffuse area light sources results ........................................43

Figure 27: Test case 5.3: Simulation of photometric area light sources results...................................43

Figure 28: Test case 5.4: Luminous flux conservation results.......................................................4

Figure 29: Test case 5.5: Directional transmittance of clear glass $(\mathrm{T})$ results ..................................45

Figure 30: Test case 5.6 geometry \& light source descriptions (CIE TC 3.33, 2005).........................46

Figure 31: Test case 5.6 measurement point description (CIE TC 3.33, 2005) ..................................47

Figure 32: Test case 5.6: Light reflection over diffuse surfaces $(50 \times 50 \mathrm{~cm})$ results ............................47

Figure 33: Test case 5.6: Light reflection over diffuse surfaces $(4 \times 4 \mathrm{~m})$ results.....................................48

Figure 34: Test case 5.6: Light reflection over diffuse surfaces $(50 \times 50 \mathrm{~m})$ results .............................48

Figure 35: Test case 5.7 geometry and measurement point description (CIE TC 3.33, 2005) ..................49

Figure 36: Test case 5.7: Diffuse reflection with internal obstructions results ..................................50

Figure 37: Test case 5.8: Internal reflected component for diffuse surfaces results ..............................51

Figure 38: Test case 5.9 \& 5.10 geometry \& measurement point description (CIE TC 3.33, 2005) ...........52

Figure 39: Test case 5.9: Sky component for a roof unglazed opening results ....................................53

Figure 40: Test case 5.10: Sky component for a roof glazed opening results ...................................53

Figure 41: Test case 5.11 \& 5.12 geometry \& measurement point description (CIE TC 3.33, 2005) .........54 
Figure 42: Test case 5.11: Sky component and external reflected component for a façade unglazed opening results

Figure 43: Test case 5.12: Sky component and external reflected component for a glazed façade opening

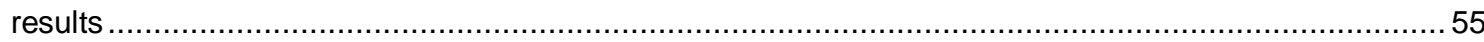

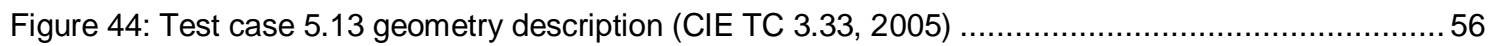

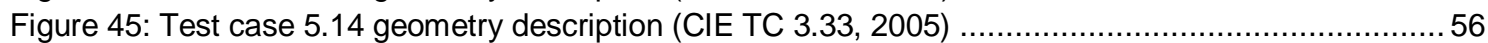

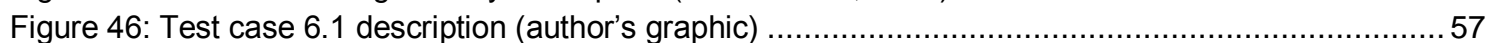

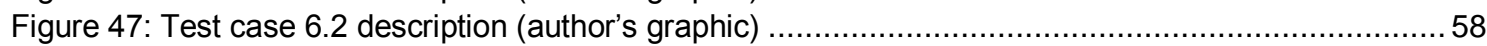

Figure 48: Test case 6.9 results; 3ds Max Design false-colour illuminances......................................62

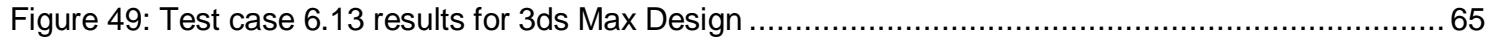

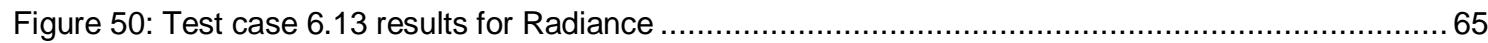

Figure 51: LBNL June 172010 \& January 152011 direct normal radiation \& workplane sensor 5 errors.. 74

Figure 52: LBNL December $29^{\text {th }} 2010$ direct normal radiation and workplane sensor 4 errors ................. 74

Figure 53: LPBS August $25^{\text {th }} 2011$ direct normal radiation and workplane sensor 1 errors.................... 82

Figure 54: LBNL 71T test-bed facility ceiling \& wall sparklines - 3ds Max Design ............................... 95

Figure 55: LBNL 71T test-bed facility ceiling \& wall sparklines - Radiance .......................................96

\section{Equations}

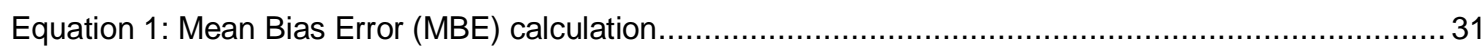

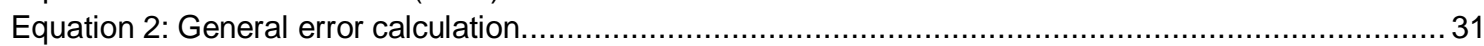

Equation 3: Equation for setting -ar parameter in Radiance (Mardaljevic, 2003) .............................. 94 
This thesis describes the process of building a comprehensive daylight simulation validation dataset for a range of complexity in lighting, but particularly daylighting, scenarios. While many datasets for this purpose exist, they are scattered, occasionally expensive, or contain data that necessitate methods unusable by many daylight simulation programs. This study begins to bring together a universal source for program neutral daylight simulation software validation data. Throughout this process the need for daylight simulation software validation is examined, particularly for further validation in complex "real world" scenarios.

The thesis builds on previous work by the author in validating 3ds Max Design for daylight simulations. The previous work (Osborne, 2009) aimed at building trust in 3ds Max Design's ability to predict internal daylight illuminances, and at its potential to significantly improve accuracy and reduce simulation time for complex light path simulations via its ability to use forward raytracing in conjunction with the widely used backward raytracing method. The previous work was based on Christoph Reinhart's 2009 experimental validation of 3ds Max Design and Daysim, a Radiance-based simulator, which showed a high level of accuracy for both software packages. These studies critically demonstrated that the use of a large number of data points - minute by minute measurements over several days - can potentially 'average out' the errors introduced by simplified sky models.

The author's previous validation work looked to make significant speed and accuracy gains within 3ds Max Design by using a global illumination (Chaos Software Ltd., 2007) simulation method available within 3ds Max Design that had not previously been validated. The research stemmed from comments in the program's supporting documentation that stated both of its raytracing algorithms are physically based, and that they could be used in conjunction with each other to produce much faster rendered image results. The algorithms are backwards raytracing (called Final Gather (FG) by 3ds Max Design) and forwards raytracing (confusingly called Global Illumination (Gl) in 3ds Max Design, a term usually referring to all bounced light in a scene regardless of how it was calculated).

The mental ray ${ }^{\circledR}$ renderer from mental images ${ }^{\circledR}$ is a general-purpose renderer that can generate physically correct simulations of lighting effects, including ray-traced reflections and refractions, caustics, and global illumination. (Autodesk, 2011)

Global illumination enhances the realism in rendered images by simulating all light interreflection effects in a scene (except caustics). It generates such effects as "color bleeding," where for example, a white shirt next to a red wall appears to have a slight red tint.

The mental ray renderer offers two distinct toolsets for achieving global illumination: photon tracing and final gathering. The primary difference between the two is that photon tracing goes from the light source toward the ultimate illuminated target (taking bounces into account), and final gathering goes the opposite way: from the illuminated surface toward the light source. You can use either of these toolsets separately, or combine them for optimal rendered results. (Autodesk, 2011)

This speed increase is due to the fact that when using forward raytracing, known as 'photon tracing' or 'Gl' in Autodesk 3ds Max Design, there is no 'searching' for a light source as is the case for backwards raytracing. Forward raytracing starts at the light source so is able to trace light quickly from its source through complex light paths. Backwards ray tracing starts at the eye of the observer or measurement point and 'finds' the light sources in the scene. For complex light paths, backwards raytracing 'searching' for a light source as small as the pin-point of the sun in the sky can take a near infinite number of rays to complete the calculation. 
This early study had a number of important findings, including that for a simple test cell in clear sky conditions, the use of Gl in simulation can produce accurate results. For cloudier or more turbid sky conditions, accuracy fell away sharply. This was due to Gl's ability to very quickly and accurately simulate direct light, such as sunlight, but its inability to cast forward rays from diffuse sources, such as skylight. These diffuse sources could only be simulated via Final Gather, the backwards raytracing engine, which when used with GI was limited to primary rays thus excluding any bounced light from the sky. This ultimately ruled it out as a viable daylight simulation method, pending the ability to use multi-bounce Final Gather in conjunction with GI, which would in turn eliminate most of the speed gains but potentially improve accuracy.

Other key findings of the study included significant errors in the Perez sky model for times of low sun elevation, which have been previously documented (Kleindienst, Bodart, \& Andersen, 2008), as well as significant reductions in the time required for simulations even without the use of Gl in backwards raytracing only configurations. These time benefits can likely be primarily attributed to 3ds Max Design's native ability to fully utilise modern multi-core CPUs and large memory resources, however it is thought that its raytracing algorithms are somewhat more efficient.

The study also made use of a dataset that was unable to fully test the effectiveness of the simulator's ability to deal with complex light paths. Crucially, 3ds Max Design is only one of many physically based daylight simulators available, with many more arriving on the market as time passes. In this study a number of existing, program or software neutral, validation datasets of varying complexity are described, along with their strengths and weaknesses, for the testing of such software. This leads to the proposal of a comprehensive validation dataset suite that includes a complex component. The proposed suite is then put through a validation study (after the individual validation methods for these datasets are determined). Key areas where modelling errors can occur are examined; with their relevance for both the validation process and their significance to designers and software developers highlighted. The findings of each dataset's validation have their own chapter documenting their significance

This introductory chapter begins with an explanation of what complexity means to daylight simulation, and why it is necessary to simulate daylighting scenarios that are highly complex. Following this, it looks at what previous validation work has achieved in the simulation of complex daylight systems, and what has so far been missing and therefore justifies further validation work in the field. The aim and scope of the study can then be set out, with the chapter concluded by an overall report outline.

\subsection{What is "complex" and why simulate it?}

Simple lighting scenarios are often met with simple lighting calculations; rules of thumb are significantly quicker and easier than simulation. Some, like the window-head-height rule of thumb, which relates the height of a window to the depth of the room that can expect good daylight, have been recently shown to give accurate predictions of good daylighting in simple side-lit spaces (Reinhart, 2005; Reinhart \& Weissman, 2012). Simulations are more likely to be used by designers to test complex architectural lighting scenarios where rules of thumb don't apply.

Most validations look at simple cases, and "trust" that the same level of accuracy will carry over to more complex simulations. This leads to a disconnect between the designers' expectations of the simulation software's accuracy and the final accuracy of their results. It is consequently important that validation be carried out not only for simple cases; but for all levels of complexity for which the software is likely to be used. The discrepancy between designers' expectations of software accuracy and its true accuracy can be further compounded as the software often comes with a starting point or set of simulation parameters derived from these simple tests. These simple tests are not what designers are simulating in practice; rather, they are simulating complete buildings and shading devices. It is therefore also important that designers can be confident that there is a valid starting point for their more complex real-world lighting scenarios. Validation is a key method in determining these starting parameters. 
Complexity in daylight simulation arises from a number of different factors. There are a...

...large range of advanced daylighting systems now available to the building profession. Some of these systems are still in the development or prototype stage and some systems are architectural concepts rather than products. (Ruck, et al., 2000)

Figure 1 below shows a number of modern daylighting systems that are difficult and impractical to simulate via traditional means such as raytracing or radiosity engines; they require additional techniques, some of which are discussed in the following sections. The systems are, from top left to bottom right: light pipes \& solar tubes, prisms with venetian blinds, anadolic ceilings, prismatic panels, laser-cut panels, and fish systems.

Figure 1: Examples of highly complex daylight systems (Ruck, et al., 2000)
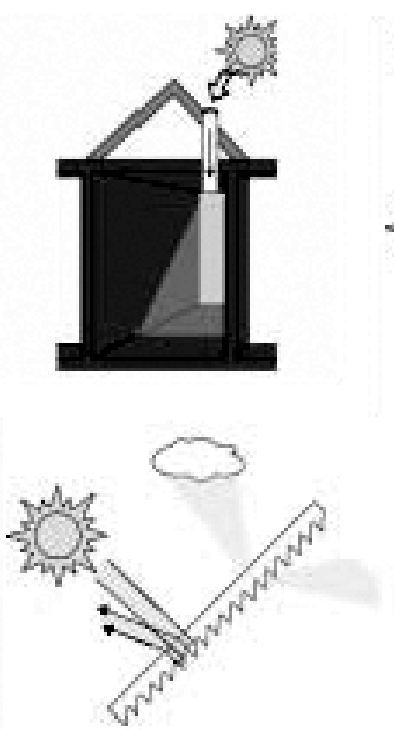
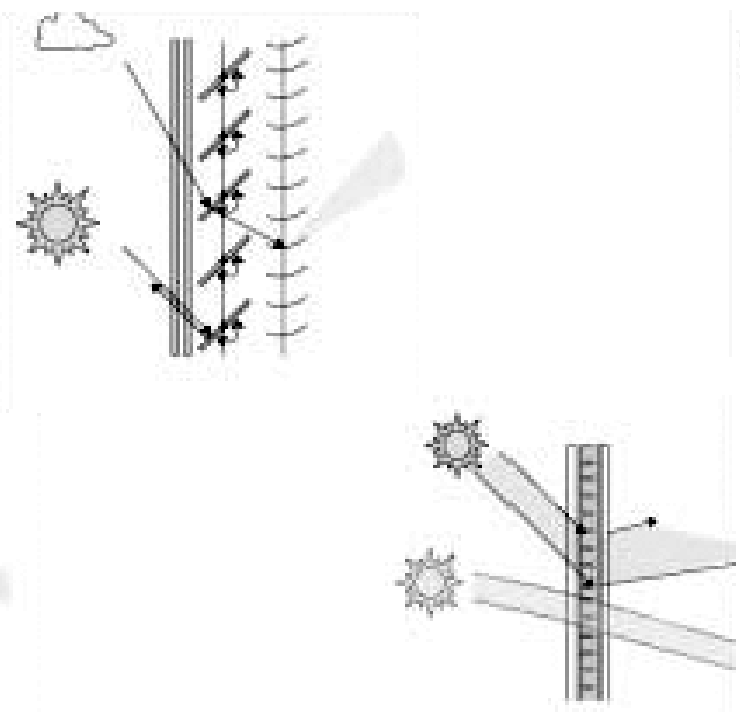
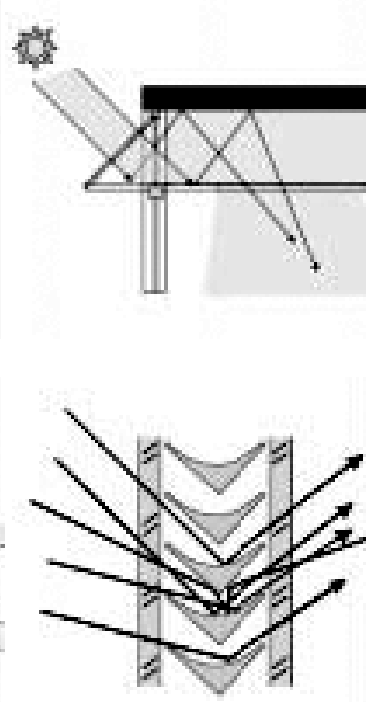

Highly detailed models have a much higher potential for errors than lower detailed ones due to the increased ability for small errors to compound. This is made worse when there are highly detailed objects near the primary light source; such is the case with venetian blinds near a window. Unless the modelled geometry is exceptionally accurate large errors will occur initially and be compounded each time the light path interacts with the element. Complex material definitions also suffer from similar effects, where anything other than diffuse materials including specular reflectors are difficult to measure and model with a high degree of accuracy.

For this study, complexity has been broken down into several different categories for clarity. These are only partly defined by the architecture or daylight system's geometric complexity. This is because the light path, being the path between illumination sources and receiving surfaces, for a diffusely painted atrium and a diffuse blind are quite similar. There are likely one or two interactions with glazing, followed by one or two bounces off of a diffuse surface such as a wall or blind fin or blade. However, a diffuse blind and a specular, light redirecting blind are very different in terms of the simulation strategies needed respectively. The categories are:

- $\quad$ Basic - First principle tests; not often used by practitioners; but which this study will later show probably should be;

- Simple - Single or possible multiple opening rooms with low detail geometry and simple uniform diffuse materials;

- Complex - High likelihood for multi-bounce light paths to illumination source and primarily uniform diffuse or simple specular materials such as glass panes;

- Highly complex - Multi bounce, complex geometry with highly specular or light redirecting materials such as curved mirror elements. 
The Radiance light simulation software package, one of the most widespread, has undergone comprehensive validation over a period of more than twenty years, both in its raw form and as part of more targeted simulation suites (Grynberg, 1989; Mardaljevic, 1995; Khodulev \& Kopylov, 1996; Mardaljevic, 1997; Mardaljevic, 2000; Reinhart \& Herkel, 2000; Mardaljevic, 2001; Reinhart \& Walkenhorst, 2001; Li, Lau \& Lam, 2004; Mardaljevic, 2004; Maamari, Andersen, de Boer, Carroll, Dumortier, \& Greenup, 2006; Reinhart \& Andersen, 2006; Reinhart, 2009). These studies cover many of the different scenarios likely to be encountered in daylight simulation. This has led to Radiance being known as one of, if not the most trusted light simulation software packages available, particularly due to Mardaljevic's 1995 study using the "gold standard" BRE-IDMP dataset with real sky conditions. However, 3ds Max Design, a popular architectural visualisation tool that recently gained daylight analysis features, has been reliably validated only once; by Reinhart in $\mathbf{2 0 0 9}$ for one location in Ottawa. The Perez sky model which it uses has been tested only for a few locations: Berkeley California, where the data it was built from was recorded, Japan, Great Britain and Switzerland (Ineichen, Molineaux, \& Perez, 1994) as well as in Reinhart's 2009 Ottawa validation and for New Zealand by Pohlen et al in 2006. Further, 3ds Max Design's single validation was entirely based on empirical, measured data. While it is likely that some analytical testing has occurred during its development, there has been no publicly available analytical verification of the software's ability to simulate light at the most basic level, which could serve as an important step in increasing trust in its results. Additionally, the majority of validation studies make use of simple experimental scenarios, which allows users to question whether simulation results for more complex real world architectural scenarios are reliable daylight performance indicators.

Radiance, partly due to its free and open development and relatively easy customisability, is currently at the forefront of research into methods of dealing with complexity in daylight simulation. One key area is in the measurement and implementation of Bi-directional Scattering Distribution Functions (BSDFs) as mathematical representations of complex light redirecting systems, such as specular blinds or holographic elements. These eliminate the need to accurately model the geometry and materiality of complex systems and thus shorten the light-path, from potentially many interactions to one mathematical step. They are inherently much faster to simulate, and have been shown to produce accurate results (Konstantoglou, Jonsson, \& Lee, 2009; Ward, Mistrick, Lee, McNeil, \& Jonsson, 2011). However, to date many popular daylight simulators are unable to implement BSDFs. Further, some complex systems are entirely arbitrary, such as an atrium, and can therefore never be consistently replaced with a mathematical model in a simulator. This type of light path complexity will always be prevalent in daylight simulations of buildings. Consequently it is still important to assess the ability of current daylight simulators' abilities to deal with these complex systems and light paths using their existing methods.

Only two previous studies are known to have examined 3ds Max Design's ability to predict internal illuminances, including the author's, compared to the many that examine the Radiance light simulation software. Reinhart's 2009 validation was important in several factors; first and foremost that, for a simple room with a variety of window conditions, 3ds Max Design's lighting analysis tools proved comparably accurate to the current "gold standard" lighting simulator Radiance. This validation also provided two other key pieces of information; that the Perez sky model works for another location outside of Berkeley California where the data it was designed from originated, and that over a large number of time samples, the large up and down errors of an intermediate sky with moving cloud patterns can be effectively averaged out. This is significant as it provides a basis for future validation of software where a more complex sky model is not available. It is noted however that being validated by only one dataset does not build a significant enough amount of trust in the software.

Significant investigation has also been undertaken for both daylight simulations and scale model investigations into the effects of the many variables involved in creating a daylight simulation model. Typically this research focuses on one or two of a few standard areas such as materials or sky models. There are numerous studies for both artificial skies and mathematical sky models like the CIE Standard Skies, but comparatively few that look at the Perez sky model. These types of investigations are useful for separating and estimating the error contributions from the individual aspects of a model. This study has the opportunity continue this practice in the context of the datasets chosen for the final validation suite, however with a much broader spectrum of modelling variables. These include, in addition to the overarching theme of complexity, materiality, the Perez sky model and the corresponding real weather data inputs, model geometry, and sensor location. 
In creating any dataset it is important to assess its viability for use in validation of simulation software; it must be thoroughly checked for measurement errors. A dataset must also undergo initial testing to provide minimum accuracy targets for future validations using it. This study therefore includes a significant validation of two popular daylight simulation programs using its datasets; and attempts to separate the resulting discrepancies between the dataset and the software. This validation also provides data on simulation scenarios that have not been significantly tested previously: "real world" scenarios where daylight simulation would most likely be used. Traditionally the reasons for validating any simulation method or program are three-fold; first and most important initially, is to identify any weaknesses or errors in the program. This allows users to avoid any areas where the program may be performing incorrectly, and highlights areas that need work from developers. The second is to remove one of the key barriers to the use of simulation in practice; a lack of trust in simulation results. Independent study as well as more widespread use will aid in increasing this trust. And finally, it is vital to provide a valid starting point for the users of the program, in this case various building or daylight system designers. It is important that new users of any simulation program be given instructions or at minimum an example of a valid or correct method to use. Through the validation process an example of a valid starting point for designers' simulations can be produced, as well as a set of recommendations on how best to achieve reliable simulation outcomes in as short a timeframe as possible. Knowing how much modelling is required and how close results are likely to be to reality based on the information a designer has will greatly improve the relevance and influence of daylight simulations in early design stages.

Recent research into the use of daylight simulations has shown that while previously there has been a high level of doubt about the accuracy of available simulation programs, those concerns have been fading (Reinhart \& Fitz, 2006). It is suggested by Reinhart \& Fitz in their 2006 survey that this increase in trust may be due to the widespread use of Radiance and Radiance-based software for daylight simulation and the considerable amount of validation work that has focused on it. However, with the recent addition of the Lighting Analysis Assistant to Autodesk's 3ds Max Design software, there is a relatively new simulator available, albeit one which has been subject to very little independent testing. While 3ds Max Design has been well validated once, this is not comparable to the comprehensive set of validations undertaken with Radiance. Many designers already use 3ds Max to generate synthetic images for presentation purposes, and therefore it can reasonably be assumed that many might also begin using this tool for lighting simulation, if they felt they could trust it. Consequently, it is important for the Lighting Analysis Assistant system to be validated to ensure trust in its simulation results.

An increase in trust is likely to support an increase in the use of daylight simulation in design; and therefore more comfortable and energy efficient buildings are likely to result. However, the primary barrier to the use of daylight simulation is currently time constraints (Galasiu \& Reinhart, 2008). With most projects having tight schedules, designers simply cannot afford the multiple-day or even overnight timeframe that daylight simulations can take for single design variant calculations. Additionally, in 2008 Galasiu \& Reinhart found that designers predominantly used simulations late in the design process; as more of a design verification rather than an outright design tool. This is significant because by disregarding daylight in the early design stages, the daylight design is surely less able to be fully resolved, and the final design is destined to suffer.

... as demonstrated by specialists in design methods, decisions taken in those moments can determine the success or failure of the end product. (Hari, 2001)

It is therefore important that the simulation packages available to designers are not only accurate, but are able to produce those accurate results in a short timeframe conducive to an iterative design approach; a matter of hours rather than days. 3ds Max Design is innately suited for high speed simulations, as it has the built-in ability to distribute calculations simply between the multiple processor cores of modern CPUs. Furthermore, it contains an extensive set of modelling and inter-operability features which allow for large numbers of design variations to be tested with ease, in addition to animation features allowing for dynamic daylighting studies to be undertaken. This has the potential to bring daylight simulation, even in the early stages of design, to the mainstream of building design, not just for large design firms or those with a keen interest in computer science. 


\section{$1.4 \quad$ Aim}

The primary aim of this research is to produce and make widely available a suite of highly documented datasets for use in daylight simulation validation studies. The suite will contain a range of datasets that contain at a minimum some simple tests and more complex real data. In order to ensure the legitimacy of the data, the suite will be fully validated, using common daylight simulation software. Accuracy will be determined via a number of comparisons; including between analytically derived values and simulated data, the comparison of real-world measurements of internal illuminance to simulated internal illuminances from 3ds Max Design and from the current 'gold standard' lighting simulator, Radiance.

The research also serves to further the validation work in daylight simulation for the software involved; testing the viability of daylight design tools for all stages of the design process; from early sketch designs through to complex finished designs by considering the amount of time taken to complete simulations and the relative accuracy at differing levels of complexity. The research is further intended to provide designers with confidence and build trust in the accuracy of daylight simulation tools as well as provide designers with a valid starting point and set of important parameters to consider when conducting daylight simulations in practice.

\subsection{Scope}

Much of the previous work on validating lighting simulation software has been limited to simplified situations often not encountered by the designers who use simulations in practice. This study covers a wider range of complexity in daylight simulation; including such simplified scenarios; and extends both towards the most basic and highly complex. Full year simulations of the empirically measured buildings used for the study would be preferred as recommended by Reinhart \& Herkel in 2000; however no such dataset is currently available. Instead, this study makes use of high frequency measurements for comparison with simulated results; with data recorded at least every minute for several days of differing weather conditions. This provides a sufficient number of data points for a representative comparison.

This study is for the most part limited to the simulation of internal illuminances, as they are the primary interest of the majority of designers (Reinhart \& Fitz, 2006). Daylight Factors, Useful Daylight Illuminances (UDIs) and Daylight Autonomy are all calculated from these internal illuminances, and as such are tied to them in terms of accuracy. Luminances, Glare Indices and other metrics have not been considered.

The study also excludes the potential of the use of 'daylight portals' in 3ds Max Design or the mkillum subprogram in Radiance, which create placeholder light sources at window openings to improve the accuracy of simulations as well as reducing the time taken to complete them. These fall under a class of more advanced techniques not available in all simulators which are outside of the aims of this research. For the same reasons it does not explicitly examine some of the newer experimental methods of simulating complex weather conditions or light paths; such as HDRI skies, BSDF's or importance sampling, but the way in which the datasets have been constructed is intended to allow for their potential inclusion in future. 


\subsection{Report Outline}

This report has been structured to enable the reader to follow the course of this research easily.

Chapter 1 - Introduction contains an introduction to the research, the previous work on which it is built and the reasons why it has been undertaken; as well as the research aim, the scope, and this outline of the report structure.

Chapter 2 - Method provides a research hypothesis, then examines the current standard in daylight simulation validation datasets, and recommends the use of an orthogonal validation procedure. It then proposes and describes a number of datasets to be included as part of an orthogonal validation suite, as well as the method by which they will be modelled and simulated.

Chapter 3 - Quality Assurance discusses the many potential sources of error in daylight simulations; and compares the accuracy of input data to the accuracy of simulated predictions. From this it then provides this study's definition of accuracy and how it will be measured.

\section{Chapter 4 - Validating 3ds Max Design and Radiance against the CIE 171:2006 test cases} documents the overall and individual test case results of the first validation dataset in the proposed suite; the CIE 171:2006 test cases. Based on these findings, it discusses whether the test cases inclusion in the validation suite is justified.

Chapter 5 - LBNL 71T test-bed facility results examines 3ds Max Design and Radiance's ability to predict internal illuminances for a simple, single-opening test cell with and without blinds.

Chapter 6 - Université de La Réunion LPBS results examines the internal illuminance predictions of 3ds Max Design and Radiance for a simple office and complex classroom in a tropical climate.

Chapter 7 - Conclusions \& discussion provides the overall conclusions of the study and discussion on their significance to the field of daylight simulation.

Chapter 8 - Future work highlights areas where this research and the daylight simulation field in general needs to continue development for the improvement of simulation accuracy and worth to designers. 

This chapter contains the overall method for producing and validating the proposed suite of datasets. Firstly section 2.1 provides the research hypothesis. It then provides a description of the potential datasets available for inclusion in the suite in section 2.3, in the context of the overarching "orthogonal" validation process proposed by Mardaljevic in 2002 and described in section 2.2. This is followed in section 2.4 by the validation methods for each dataset, including appropriate simulation parameters for the software used. A comprehensive study on quality assurance is later described in detail in chapter 3; identifying key areas of the datasets which may produce error. This aids in producing a definition of accuracy within the report; given later in section 3.7.

\section{$2.1 \quad$ Hypothesis}

The research hypothesis is that it will be possible to create a suite of daylight simulation validation datasets that tests a spectrum of simple to complex daylighting scenarios likely to be encountered by designers in practice, and that is able to be used in any daylight simulation software package. The suite will provide opportunity for the addition of more advanced simulation techniques as they are developed and included in simulation software.

\section{$2.2 \quad$ Methodology}

\subsubsection{Orthogonal validation}

This study proposes a suite of datasets for use in "orthogonal" validations of daylight simulation software. This method of validation is proposed by Mardaljevic in his description of the BRE-IDMP dataset; which showed very good agreement between the Radiance simulation package and measured data using measured sky luminance patterns (Mardaljevic, 2002). The procedure, shown in figure 2 below, suggests that the ideal way to validate a daylight simulation program is against the BRE-IDMP dataset; or if that is not possible then to compare it to a program that has undergone such a validation. In the case of this study; like 3ds Max Design, typical daylight simulators are unable to natively use measured sky luminances from a sky scanner. It will therefore be orthogonally validated against Radiance, using the proposed dataset suite.

Figure 2: Orthogonal validation procedure (Mardaljevic, 2002)

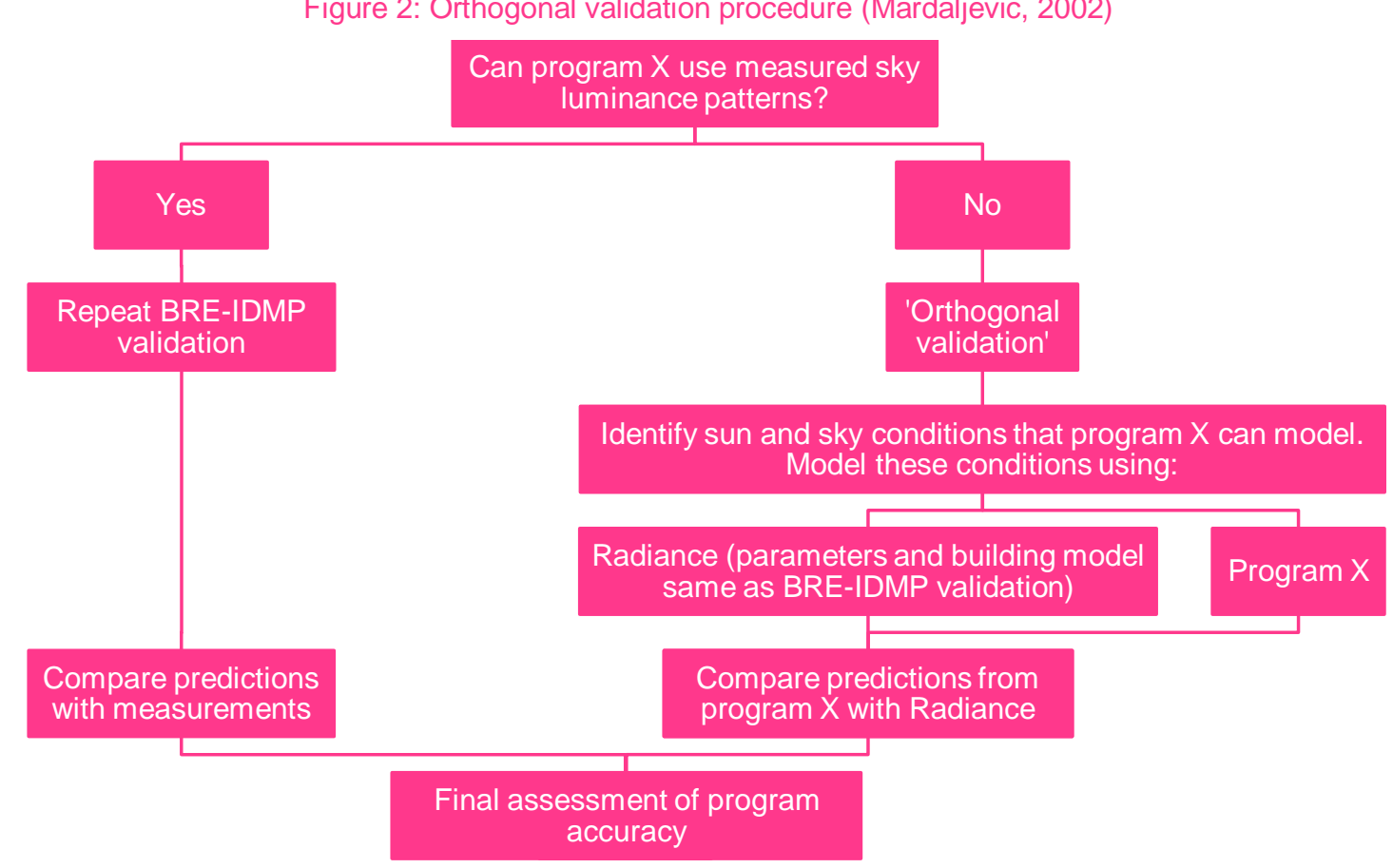


Validations involving sky-scanner derived sky luminance patterns, whilst having the benefit of being closer to real sky conditions, are less relevant to the users of daylight simulation software - designers. Due to the high cost of producing such data, designers are much more likely to use more inexpensive and widespread data such as the US Department of Energy's (USDOE) Typical Meteorological Year (TMY) EnergyPlus Weather (EPW) data. This data already sees widespread use for thermal simulation and is suited for use in daylight simulation as well. However; sky models making use of this data can only rely on the Direct Normal and Global Diffuse elements; there are no detailed directional qualities of the sky luminance included.

Using this method affords an opportunity to construct a much more comprehensive suite of data sets, from multiple distinct sources. The current standard for energy simulation software validation is the International Energy Agency's (IEA) Building Energy Simulation Test (BESTEST) cases. Produced by Ron Judkoff, these are an:

... integrated suite of building energy analysis tool tests involving analytical, comparative, and empirical methods... for evaluating, diagnosing, and correcting building energy simulation software. (International Energy Agency, 2008)

The third objective of the BESTEST project is:

To create and make widely available high quality empirical validation data sets, including detailed and unambiguous documentation of the input data required for validating software, for a selected number of representative design conditions. (International Energy Agency, 2008)

Much like BESTEST, this project therefore aims to produce, and make widely available, a validation dataset suite for daylight simulation that mirrors the simulation tasks that are commonly used in the field. This not only provides a benchmark for software developers to check their software versions against; but also allows designers to assess how close to reality their simulations using simplified sky models and weather data are likely to be. The final dataset suite should aim to fill the matrix presented in table 1 below. The initial datasets proposed to be included are discussed in the following section, along with the importance of each element in the matrix.

Table 1: Dataset matrix

\begin{tabular}{|c|c|c|c|}
\hline Complexity & Location & Weather & Dataset \\
\hline Basic & $\mathrm{N} / \mathrm{A}$ & $\mathrm{N} / \mathrm{A}$ & $?$ \\
\hline \multirow[t]{6}{*}{ Simple } & \multirow[t]{3}{*}{ Perez 'home' } & Clear & $?$ \\
\hline & & Mixed & $?$ \\
\hline & & Overcast & $?$ \\
\hline & \multirow[t]{3}{*}{ Perez alternate } & Clear & $?$ \\
\hline & & Mixed & $?$ \\
\hline & & Overcast & $?$ \\
\hline \multirow[t]{6}{*}{ Complex } & \multirow[t]{3}{*}{ Perez 'home' } & Clear & $?$ \\
\hline & & Mixed & $?$ \\
\hline & & Overcast & $?$ \\
\hline & \multirow[t]{3}{*}{ Perez alternate } & Clear & $?$ \\
\hline & & Mixed & $?$ \\
\hline & & Overcast & $?$ \\
\hline
\end{tabular}




\subsection{Available datasets}

This section discusses a number of datasets that were considered for inclusion as part of the suite for daylight simulation validation. Each has been given their own subsection.

\subsubsection{BRE-IDMP Validation Dataset}

The BRE-IDMP daylight simulation validation dataset, created by John Mardaljevic, is widely considered one of the best sources for validation data available. It utilises data recorded at the Building Research Establishment (BRE) in Garston, United Kingdom as part of the International Daylight Measurement Program (IDMP). The dataset contains simultaneous measurements of sky luminance patterns in 145 'patches', direct normal and vertical illuminance in each of the cardinal directions, temperature and humidity, and internal illuminance in two full-size mock offices (Mardaljevic, 2001). The data is of a very high quality; has been rigorously checked for errors and has known tolerances.

The BRE-IDMP dataset is therefore more than adequate to carry out a successful validation study of a daylight simulator; however on its own it fails to meet the aims of this project in a number of ways. Firstly; it does not fully satisfy the wide range of complexity that simulations are likely to be used for in practice. While it does contain data for both a simple office with a single opening and a complex glazing system, it does not extend far enough into the basic, first principle tests required of a comprehensive validation. While this leaves it able to be used in conjunction with additional basic tests, it also would require the addition of further variations in window complexity.

Secondly, due to the nature of the datasets sky measurements, it is unable to be used in a large number of daylight simulation software packages. While the 145 and higher patch-based sky models are arguably the most accurate available, due to the rarity of compatible data it remains unimplemented in most software. While it is theoretically possible for simulators with the capability to model sky luminance via High Dynamic Range Images (HDRI) to use the data, this is still an emerging technique for sky modelling and requires significantly more testing. Furthermore, the dataset only contains data for one location, the UK, and it is desirable to check sky model accuracy at numerous locations around the world. Including further locations from within the IDMP may be a relatively straightforward remedy for this.

Finally, the dataset does not fully meet the fundamental criteria of being widely available. Whilst being free for use in the validation of non-commercial software, its price for commercial software validation is prohibitive. Whilst it is understandable that the costs of creating such a dataset are high; it is felt that the potential benefit for building users globally outweighs this, as having better free and commercial building simulation software will inevitably provide for better buildings.

The BRE-IDMP dataset has been used for at least one known validation, of the free Radiance light simulator, with positive results. As the validation showed Radiance to accurately reproduce internal illuminances, Mardaljevic proposes that any validation study that cannot make use of the BRE-IDMP dataset instead undergo an "orthogonal" validation with Radiance, as discussed in the previous section. 
Developed under the CIE Technical Committee 3-33, the CIE 171:2006 test cases are an extensive suite of empirically and analytically derived experiments which have been designed to test lighting simulation software accuracy at a basic level.

The objective of this report is to help lighting program users and developers assess the accuracy of lighting computer programs and to identify their weaknesses. (CIE TC 3.33, 2005, p. 7)

These test cases are crucial in providing the desired level of trust in simulation software, but also aid in preventing unwarranted trust in simulations. By breaking each aspect of simulation into separate test cases, problems in the calculation algorithms or software itself are easily identified. It is therefore highly recommended, by the BESTEST process and the authors of the CIE 171:2006 document, that all new simulation software undergo some sort of similar testing. Test cases such as these can also be highly influential in the creation of a starting point for designers; by highlighting where certain simulation parameters begin break down or become overly expensive in terms of simulation time over accuracy. However, while the test cases serve as an important initial step in a validation, they do not provide many important aspects necessary to fulfil the aims of this research. It is documented that the test cases "... should be considered a first step towards an efficient methodology" (CIE TC $3.33,2005)$.

Firstly, while the targeted individual aspects of simulation are parts of complex light paths in more complex scenarios, these need to be tested together in order to make a true assessment of overall program accuracy. This can only be done through experimental testing of real world scenarios. Secondly, whilst influencing a starting point; they cannot paint the full picture of what is required for any given scenario. Starting points are better defined by tests with "typical" scenarios, and then adjusted accordingly.

Finally, a key constraint of the CIE test cases is that they do not test the highly adaptable and widely accepted Perez sky model, which is discussed later in this document. Whilst providing a large scope for testing of the legacy, and still commonly used, CIE standard sky set, these are becoming less and less relevant with the increase in use of dynamic daylight simulation. As only two of the sixteen skies in the set are commonly used, the CIE Clear and Overcast (also further discussed later in the document), this study has excluded the remaining intermediate skies.

\subsubsection{Lawrence Berkeley National Laboratory 71T test-bed facility}

Lawrence Berkeley National Laboratory in Berkeley California have for a number of years been collecting a wide variety of data at their $71 \mathrm{~T}$ test-bed facility. The facility is being used to test several aspects of building environment, including energy, thermal and, crucially to this study, daylight performance. Their significant collection of data is consequently innately suited to daylight simulation validation, as it contains all of the necessary elements for analytical testing.

The facility records internal illuminances on a minute by minute basis, suitable for validation purposes where accurate year-round data is hard to come by, for three rooms of regularly varying window conditions (see figure 3 below). Each of the rooms has a single opening, facing the equator. The facility also records accurate direct and global horizontal radiation data on the same timescale. This provides the unique additional ability to test the effect on simulation accuracy of the Perez sky model in its 'home climate', as it was formulated using data from the same location. This provides a baseline that will allow for estimations of error arising from the sky model's simulated luminance predictions in other locations. Further weather data for temperature and humidity required for the Perez sky model's haziness calculations has been provided from another facility at Berkeley, the Environment Health and Safety Meteorological Weather Station. Retrieved from their online portal, this data is recorded at quarter hour intervals, and has been linearly interpolated into minute time steps. As temperature and humidity vary relatively slowly, this has a minimal impact on accuracy. 


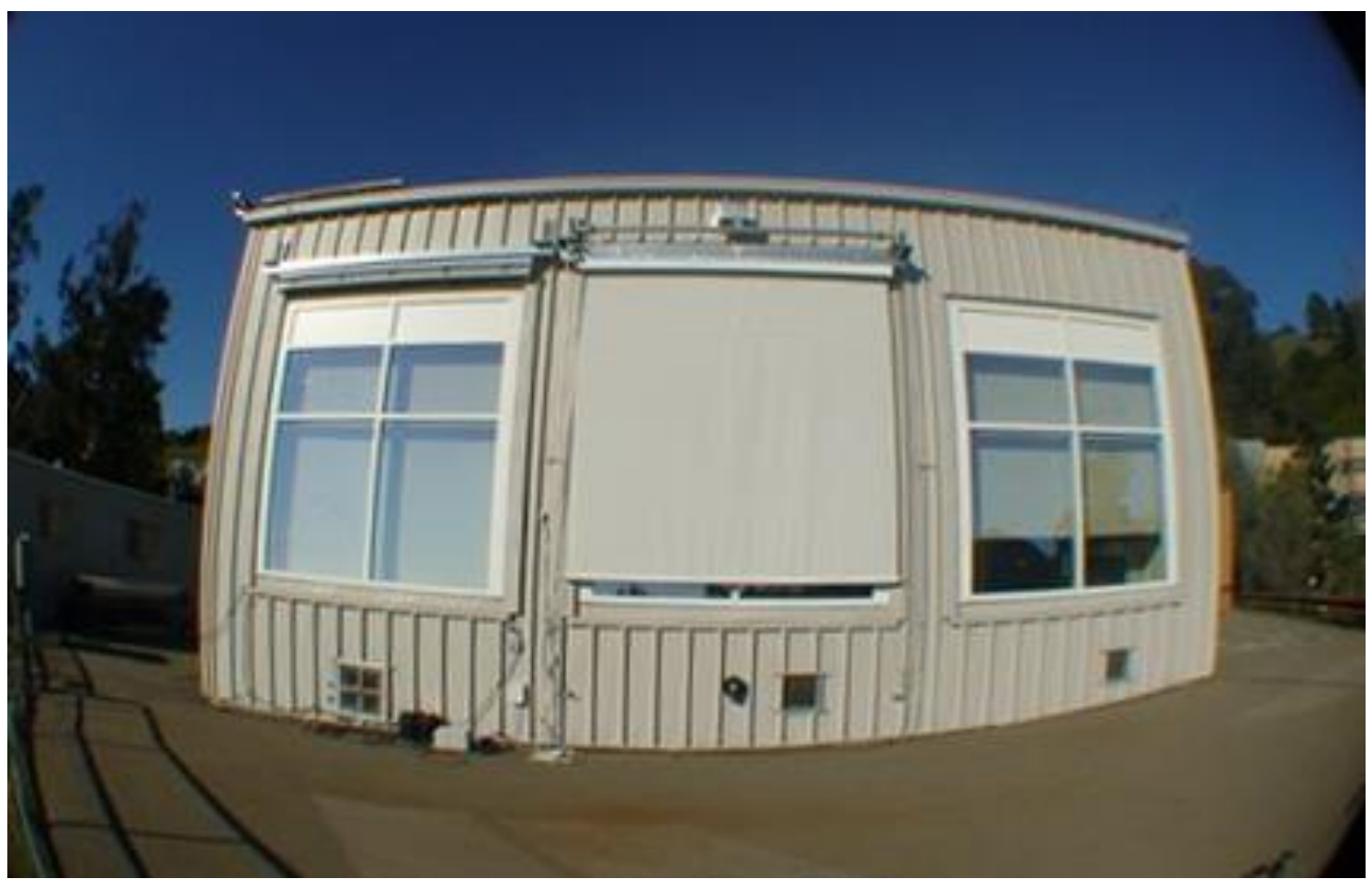

For this study, a small selection of data from the facility has been used that satisfies a number of conditions. Firstly; two window conditions were sought; a simple, unshaded window, and a more complex venetian blind system. Secondly for both of the window conditions a range of weather conditions are included; clear, mixed and overcast. This again aids in isolating sources of error, by providing indications of sky model accuracy under differing weather conditions. While the selected data does not extend into the highest of complexities encountered in modern daylight design, as the facility is also being used to test a number of high-complexity glazing systems, their inclusion in the dataset is possible at a later date.

\subsubsection{Université de La Réunion Laboratoire de Physique du Bâtiment et des Systèmes}

The Université de La Réunion's Laboratoire de Physique du Bâtiment et des Systèmes (LPBS) building, situated on the French colony Isle de la Réunion in the Indian Ocean, provides for a daylight simulation dataset that complements the LBNL dataset very well. It has many features that make it unique; firstly that it is in a tropical climate, which allows for an assessment of the Perez sky model for an as yet untested location. Also unique is that the dataset makes use of two occupied spaces, an office and a classroom, in a facility designed specifically with daylighting in mind. The building, pictured below in figure 4, has been designed in such a way that it requires no electric lighting during the day other than in the enclosed areas such as restrooms and lifts. The two currently constructed wings of the building have louvered windows on their North and South sides, with an atrium between the two with louvers to the West. 


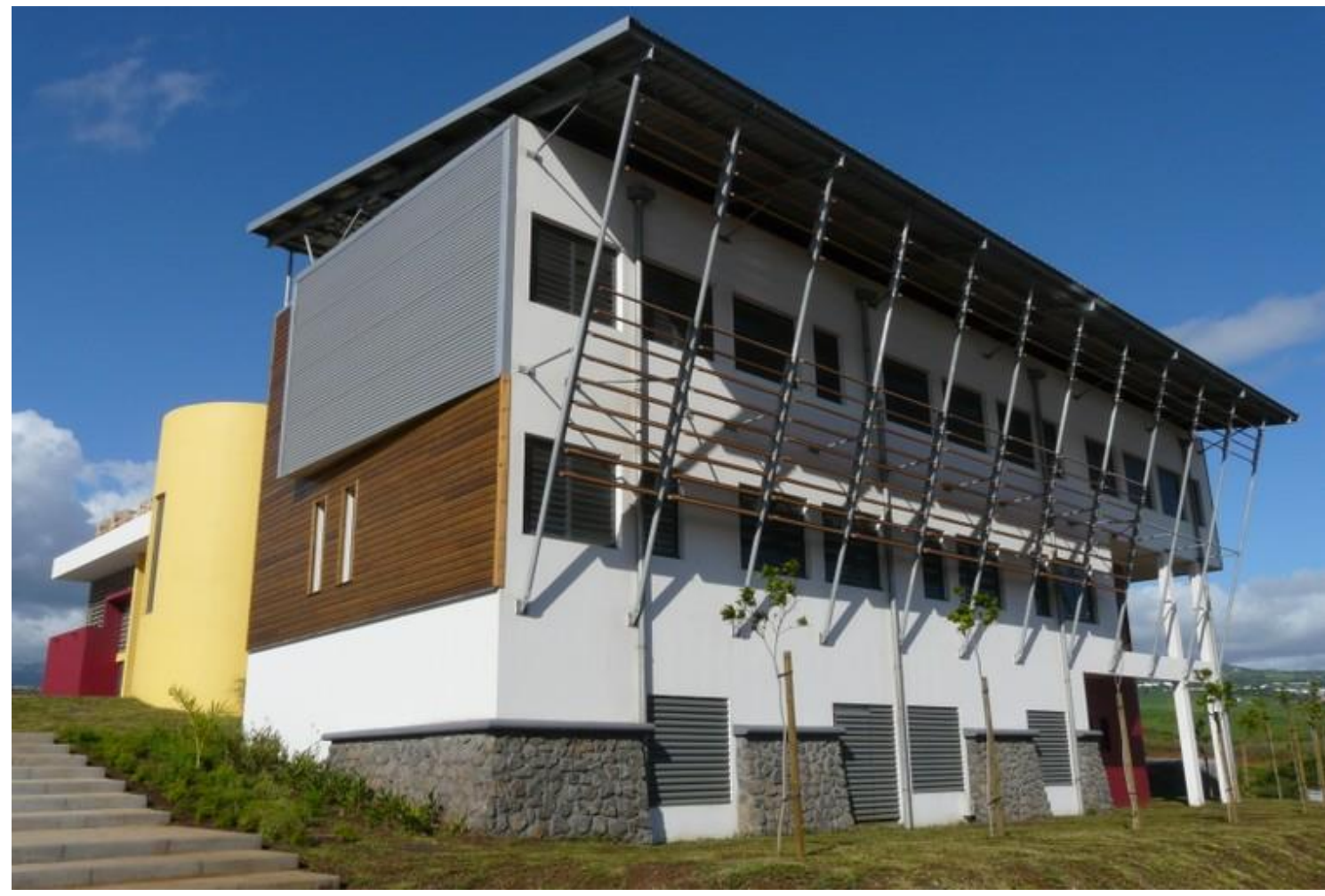

The dataset makes use of a relatively unshaded office, pictured at the left, below in figure 5 , with only a small overhang over the window. The glazing faces North, towards the equator, as it does in the LBNL 71T test-bed facility. The classroom is located on the second floor, in the South West corner of the building, and has glazing on both the South side (pictured in figure 4 above and at the right in figure 5 below) and North into the atrium. The classroom satisfies the complex condition required for the dataset suite; especially given that the majority of light entering the space has undergone one or more diffuse reflections.

Figure 5: LPBS office (left) and classroom (right) interiors (photos courtesy Shaan Cory)

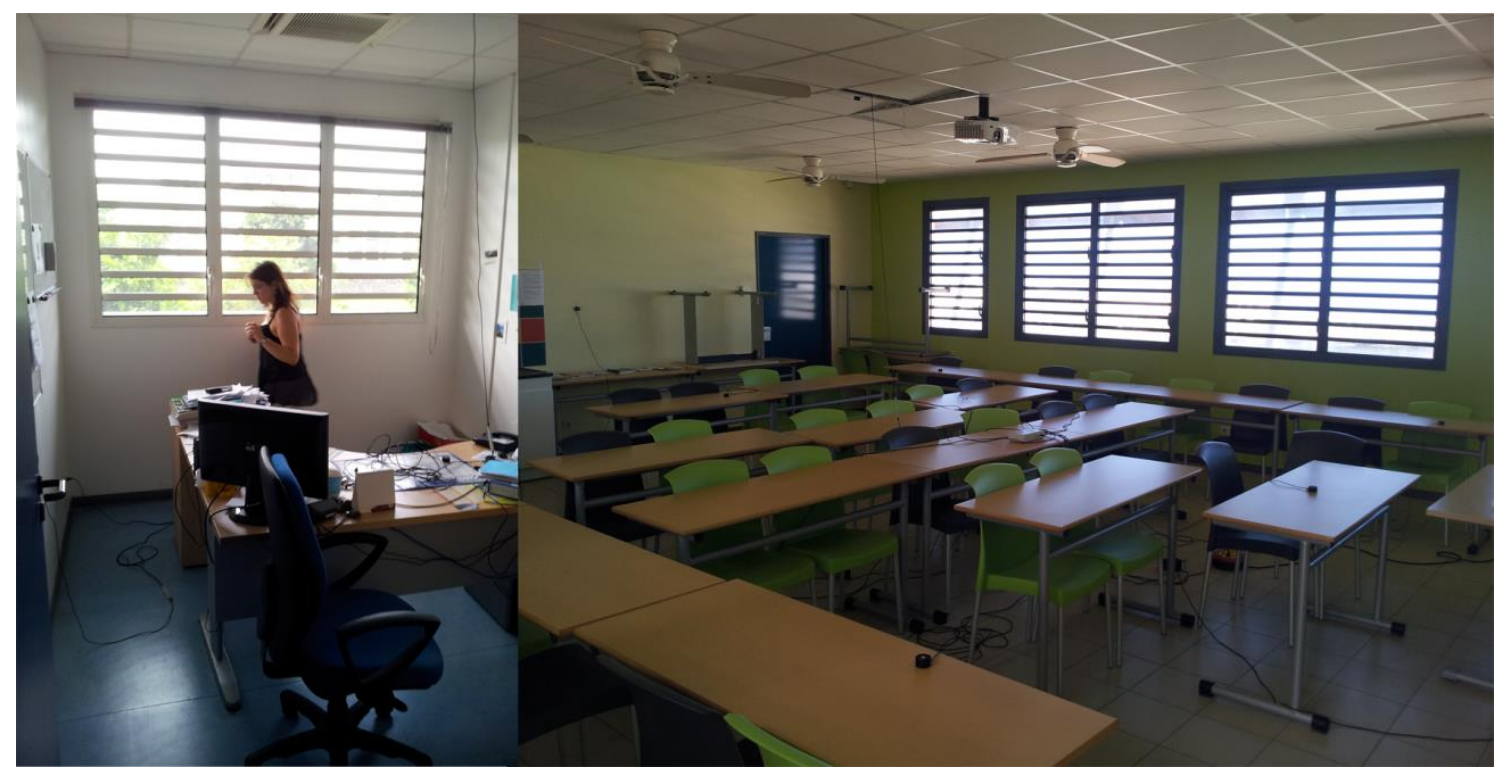

Again this dataset contains a range of weather conditions, however not as broad and not over as many days as with the Berkeley dataset. It is thought however that there is enough data to draw the necessary conclusions about the weather type's influence on simulation accuracy. All weather data was recorded at the facility's own weather station. 


\subsubsection{The validation suite}

Highly simplistic empirical tests are essential, but do not provide the level of trust of simple or complex validations. For any validation to be helpful to simulators in practice, it needs to address real-world situations; daylighting solutions that designers would like to test. The final dataset suite proposed by this study is presented in table 2 below. The matrix of datasets from basic to complex is clear, which also neatly covers multiple climates and weather conditions. While not all situations are filled, the suite should provide an acceptable cross section of conditions to fulfil the aims of this research.

Table 2: Complete dataset matrix

\begin{tabular}{|c|c|c|c|}
\hline Complexity & Location & Weather & Dataset \\
\hline Basic & $\mathrm{N} / \mathrm{A}$ & $\mathrm{N} / \mathrm{A}$ & CIE 171:2006 Test Cases \\
\hline \multirow[t]{6}{*}{ Simple } & \multirow[t]{3}{*}{ Perez 'home' } & Clear & \multirow[t]{3}{*}{ LBNL 71T Test-bed } \\
\hline & & Mixed & \\
\hline & & Overcast & \\
\hline & \multirow[t]{3}{*}{ Perez alternate } & Clear & \multirow[t]{2}{*}{ Université de La Réunion LPBS } \\
\hline & & Mixed & \\
\hline & & Overcast & Not included \\
\hline \multirow[t]{6}{*}{ Complex } & \multirow[t]{3}{*}{ Perez 'home' } & Clear & \multirow[t]{3}{*}{ LBNL 71T Test-bed } \\
\hline & & Mixed & \\
\hline & & Overcast & \\
\hline & \multirow[t]{3}{*}{ Perez alternate } & Clear & \multirow[t]{2}{*}{ Université de La Réunion LPBS } \\
\hline & & Mixed & \\
\hline & & Overcast & Not included \\
\hline
\end{tabular}

\section{$2.4 \quad$ Digital modelling and simulation method/criteria}

As the datasets were put through an orthogonal validation it was important to keep as many of the parameters between the two simulators as close as possible. This section describes the modelling and simulation method and criteria followed to ensure the utmost consistency between software, to enable worthwhile comparisons of their resulting data.

All models created as part of the validation process followed a consistent process. This process began in Google's SketchUp 3d modelling software, which had the quickest and simplest methods of creating models compatible with both Radiance and 3ds Max Design. Use of BIM or other more automated modelling tools were deliberately avoided for creating the initial validation models, as there is much greater risk for errors to occur in a modelling tool that automates some processes. SketchUp allows for simple, full control over all aspects of modelling. In SketchUp, each object in a model was created as an individual component; with a limit of one material per component. This avoided errors due to mistakes in material application and mapping. In some cases, such as with window assemblies, multiple components were grouped into larger components. If there were multiple copies of one object in a model, the components were instanced to save memory during simulation. All solid, opaque objects were modelled at their correct thickness, with no 'paper-thin' geometry to avoid problems with light leaks and incorrectly oriented surface normals. Transparent objects, such as window panes, were modelled as thin geometry and later given two-sided materials. Once all objects were modelled placeholder materials were applied that would later be replaced in 3ds Max Design and Radiance. Once the model geometry was completed in SketchUp, it was taken to the two simulators via separate methods, with care to maintain unit consistency. This involved leaving all dimensions as they were for 3ds Max Design, and converting all units by a factor of 1000 from millimetres to metres, Radiance's native unit. 3ds Max Design has the capability to natively import SketchUp models, which was utilised. The SU2RAD plug-in for SketchUp was used to export Radiance scene files. 
Once in the respective simulators' formats, the materials created by SketchUp were replaced with physically accurate ones. In the case of the CIE 171:2006 test cases, these were an array of grey materials ranging from $0 \%$ reflectance to $95 \%$ reflectance to match the respective cases' empirical data. All of these materials were pure diffuse reflectors, modelled on the 'Arch \& Design' Material in 3ds Max Design and 'Plastic' Material in Radiance, excepting any glazing materials and materials intended for testing specular reflections. For the two empirical datasets, from Berkeley and La Réunion, the majority of materials were made using measured data from a ColorMunki Spectrophotometer, manufactured by X-Rite. To ensure continuity, the measured spectral reflectances were imported to 3ds Max Design Arch \& Design materials via X-Rite's 3ds Max Design software plug-in (X-Rite Incorporated, 2008), with the resulting linear RGB reflectance values then used for the Radiance Plastic material. In some cases, such as where an object was too complicated or out of reach for measurement, reflectances were estimated by using common place-holder values, such as a reflectance of 0.2 for the foliage in the LPBS atrium.

Once all geometry and material properties were in place, the respective lighting conditions were set up. For all cases requiring light sources defined by photometry the supplied CIBSE photometric files were first converted to an IES photometric file format. This is due to Radiance requiring the use of IES format files via its IES2RAD conversion program. In order to remain consistent the same IES files were used in 3ds Max Design. However, the original CIBSE format files were also tested and the results can be seen in section 4.1.1 later in this document. Both simulators have their own methods of creating CIE general skies, however 3ds Max Design is limited to only two of the CIE general therefore only cases using the CIE Overcast or Clear sky types have been tested. In 3ds Max Design the CIE skies are produced via its daylight system using solar altitude and azimuth angles or a date, time and location. Radiance produces CIE skies via the Gensky program included in its package using the same parameters.

For the two empirical datasets in Berkeley and La Réunion, the Perez all-weather sky model was used. Again both simulators had separate methods of implementing it, with 3ds Max Design again accessing it via its daylight system and Radiance via the Gendaylit program that is developed separate to the Radiance software itself, but widely used. Both used identical direct normal and diffuse horizontal radiation values to produce their sky descriptions, 3ds Max Design reading them from a custom made minute-time-step Energy Plus Weather (EPW) file, and Radiance via a Microsoft Windows batch file.

Simulation sensor points to match either the analytical reference points in the CIE 171:2006 test cases or the light meters in the Berkeley and La Réunion datasets were first created and carefully aligned in 3ds Max Design. To ensure consistency, their positions and orientation could then be exported to a text file and formatted for use in Radiance.

There is significant confusion within the daylight simulation profession as to what simulation settings are needed for accurate simulation results. Generally, for backwards ray-tracers such as Radiance and $3 \mathrm{ds}$ Max Design's Mental Ray, the two most commonly discussed parameters are the diffuse or ambient bounces and number of rays traced per point. During the author's initial investigations into simulation parameters, a broad range of recommendations for a multitude of settings within both simulators was found. Looking solely at diffuse bounces for 3ds Max Design, this ranged from three (Krygiel, 2010) to above seven having "limited added benefit" (Autodesk, 2010). The 3ds Max Design default value of four, Reinhart's 2009 validation value of six and many others fell in between those values. Similar values were suggested for Radiance, between three (DesignBuilder Software Ltd, 2011) and eight (Ward, 1994).

However, there is no one set of values for all simulation scenarios. Finding the ideal parameters for simulating any one scene is not as easy as picking recommended values - the parameters are often co-dependant on other parameters and work in synchronously with each other to produce results. It is best then to instead provide a good starting point for designers to use; but recommend that they perform some form of convergence test to find the ideal accuracy and simulation time trade-off. This would likely start with altering the number of diffuse/ambient bounces, number of rays, and ray interpolation, looking for the point at which accuracy increases are no longer worth the extra time taken to run a simulation. 
The entire suite of datasets used in this study were simulated with identical simulation parameters; except in special cases as noted under the respective results sections of this report. These parameters were as close as possible between 3ds Max Design and Radiance. This was ensured with expert advice from both the Radiance developers and community and the 3ds Max Design developers. The parameters used for both have been listed in table 9 and table 10 in the appendices, and are the same as those used by Reinhart's 2009 validation of 3ds Max Design and Daysim; a Radiance-based simulation package. These parameters were chosen for their already proven success in that study; and due to early testing as part of this project showing that 3ds Max Design's supplied "Lighting Analysis Preset" simulation parameters often failed to provide sufficient accuracy for the CIE Test Cases, making Reinhart's parameters an appropriate first step up.

Prior to undertaking a full validation of the datasets, it is important to determine accuracy targets. The following section describes the multitude of areas where error can be introduced throughout the modelling process described above, and examines their potential for introducing error. Using this, estimates of what reasonable targets are for daylight simulations can be made. 

Many factors contribute to errors in simulations; most of which lie outside of the simulation algorithms themselves. In order to test a simulation program's accuracy, these sources need to be removed from the models being tested, and if they cannot be removed then their contributions to simulation error need to be quantified. Not only must the data used within simulations for validations be of high quality; so must the data used to compare it with.

The sections below discuss the primary sources of errors and inaccuracies in daylight simulation and how, if possible, to avoid them. From careful review of daylight modelling guides, the following were identified as key aspects of the validation dataset that had potential to have an impact on the likely precision of the simulations. These included:

- Dealing with geometry, the physical measurements of the size of the room;

- $\quad$ The size of the opening allowing daylight into the space;

- The size of the window mullions.

- The amount of geometric detail required in a simulation model;

- Dealing with materials; the physical measurements of the reflectances within the space;

- The transmittance of the glazing;

- The amount of material detail required in a simulation model;

- Dealing with sky models and their associated inputs; the potential difference between the simple, continuous sky model and potentially discontinuous real sky conditions;

- $\quad$ The precision and frequency required of the measured direct and diffuse solar radiation ;

- The precision of the associated temperature and relative humidity data also required by the Perez model to produce a sky luminance distribution;

- Dealing with sensor point placement tolerances, the precision of horizontal placement of internal illuminance sensors;

- The precision of levelness of internal illuminance sensors;

- Dealing with data discarding; when to discard or ignore data such as in direct sunlight conditions, or when a sensor is near a shadow boundary.

Each of these items has been tested for their relative sensitivity within the digital model. The goal is to document how much of an impact they can have on overall internal illuminance values. These errors, when added together, must be below a threshold that is both reasonable enough to allow the dataset to be used to test accuracy; but also tolerant enough that it is practical to obtain.

A common method of determining such an accuracy threshold is the effect of the 'error' on design predictions. This tests whether the simulated predictions are of comparable accuracy to the input data. Some validation studies also quote accuracy of $\pm 20 \%$ Mean Bias Error (MBE) as acceptable (Li, Lau, \& Lam, 2004), (Reinhart, 2009). They suggest a $\pm 5 \% \mathrm{MBE}$ is preferable if the design predictions are to have sufficient precision that design decisions can be based upon reality, not chance differences in the simulation.

As Mardaljevic's BRE-IDMP dataset has been shown to be able to produce validation results for Radiance well within this $\pm 5 \%$ range, any new dataset using real sky conditions and contains no external obstructions should attempt to at least equal its proven accuracy. However, as this study is for alternative datasets without the ability to use more detailed skies, and with potentially more complex light path scenarios, the $20 \%$ MBE limit has been used. It can then be reasonably determined that the total error contributions from each of the aspects listed above, when averaged over the measured time period, should not add up beyond $20 \%$ in order to be considered a good quality validation dataset. Further, each of the identified areas of error introduction may be ranked according to their potential error size; highlighting the key factors of the datasets. 


\subsection{Geometry}

When creating a digital model of a space it is common to use multiple sources for measurements. Using architects' CAD drawings may speed up the process; however there is always a risk that drawings don't match what is built; as changes are made in the building process and detail may not be present in the drawings. It is therefore normal practice if basing a digital model on drawings, at least to check key dimensions on site. What is unclear is just how close the model needs to be to reality: what are the key dimensions. This study examined the changes to internal illuminance values caused by changes in the dimensions of three likely key elements: 1 ) the three principal dimensions of a rectangular room; 2) the total window opening size; and 3) the mullion width and depth. These changes were made for both the simple LBNL test cell as well as the complex LPBS building, to check for any differences the effect of these dimensions on the two levels of complexity. In addition the LPBS building, being the more complex of the two, had its model detail sequentially reduced, to test for the ideal level of detail.

\subsubsection{Physical measurements - room dimensions}

Modern hand-held laser measurement tools are able to achieve a measurement precision of up to $1 \mathrm{~mm}$ in $100 \mathrm{~m}$, or $0.001 \%$, and can therefore give the internal measurements of most rooms to highly precise figures provided they are used with care. However, to account for human error in the use of even such a precise device it is necessary to estimate how the measurement device might be aligned to measure perpendicular to a wall. It is also possible that for a small room a tape measure would be used instead. It has been assumed that a reasonable maximum percentage of error in measuring with a tape measure or slightly misaligned laser is $\pm 0.25 \%$, or about $2.5 \mathrm{~mm}$ per metre.

Differences between a CAD drawing and a built space however have the potential to be much larger. In the extreme where not one, but all the walls are placed in the wrong position off the plans, they would at most be built a distance equal to their thickness further from the opposite wall on plan; in this case about $100 \mathrm{~mm}$. This is a scenario that is more likely to occur during sketch design when detailed colour and finish data is unavailable.

To test the effect of a $0.25 \%$ measurement error and a $100 \mathrm{~mm}$ shift in wall position on internal illuminances, simulations were run of both the LBNL and LPBS spaces using models constructed to measured size, $0.25 \%$ larger internal dimensions, and with dimensions $100 \mathrm{~mm}$ larger in each of the horizontal directions from the centre of the room as a worst case scenario. In all simulations the opening and glazing sizes remained unchanged, as did the measurement point locations.

Increasing the internal dimensions of a space by $0.25 \%$ had the effect of reducing the predicted internal illuminances. The window sizes were kept constant for this exercise. Thus, the effect of this was to maintain the lumen output of the light sources (the windows) but increase the surface area over which these lumens were spread - a reduction in lumens per square metre - lux. The effect was relatively small with an average reduction in illuminance of approximately $0.5 \%$, or twice the room dimension change. A maximum reduction of $3 \%$ was observed for the back-most measurement points. For the back-most measurement points the light has bounced multiple times, compounding the effect of the slightly larger space.

Increasing the internal dimensions of a space as small as the $3.7 \times 4.6 \mathrm{~m}$ LBNL test cell plan, by $100 \mathrm{~mm}$ is an increase of internal areas by approximately $4 \%$. A similar reduction in illuminance was observed. The reduction again varied according to the distance from the opening, with smaller reductions nearer the glazing and larger towards the back wall. The reductions recorded were a maximum of $2.5 \%$ at the front and $10.5 \%$ at the back. The indication of the much greater error at the rear of the room suggests that accurate physical dimensions are more important for higher complexity spaces where multiple bounce light paths dominate. The potential for errors is therefore considerable.

\subsubsection{Physical measurements - window dimensions}

Further tests were conducted on window opening size and mullion size. For the window opening, the same level of error as the internal dimensions, $\pm 0.25 \%$, was used as most openings are of a reasonable size and easy to measure. For the mullions, a much more precise measurement would be required for their relatively small thickness to achieve $\pm 0.25 \%$ accuracy, so a worst case scenario of $\pm 2 \mathrm{~mm}$ was adopted. The predicted internal illuminances for these cases were again compared to the original measured room's simulated values. 
Increasing the size of the opening(s) into a space by $0.25 \%$ had the effect of lifting the predicted internal illuminances. A $0.25 \%$ increase in the size of the opening resulted in an average increase in illuminance of $0.5 \%$, with the largest increase for an individual point being $1.5 \%$. The size of the opening in a space has about the same influence as the size of the space itself.

Measurement accuracy for mullions was shown to be significantly less influential on internal illuminances than the overall opening size. An increase of $2 \mathrm{~mm}$ in the mullion thickness produced internal illuminance predictions on average only $0.5 \%$ lower, with a maximum of $1.5 \%$ lower for one measurement point. Significantly this shows that detailed measurements of mullion profiles are generally not required, and mullions can instead be estimated to within a few millimetres.

\subsubsection{Model geometric detail}

A digital model can never contain as much detail as reality; it must always be simplified to a degree. In order to test the likely impact of simplifying model geometry, a number of tests were made using the more complex LPBS dataset. For this dataset a highly detailed digital model was originally created from architects CAD drawings, photos, and hand measurements, a render from which has been shown in figure 6 below.

Figure 6: Render of detailed CAD model of the LPBS building, without measured reflectances

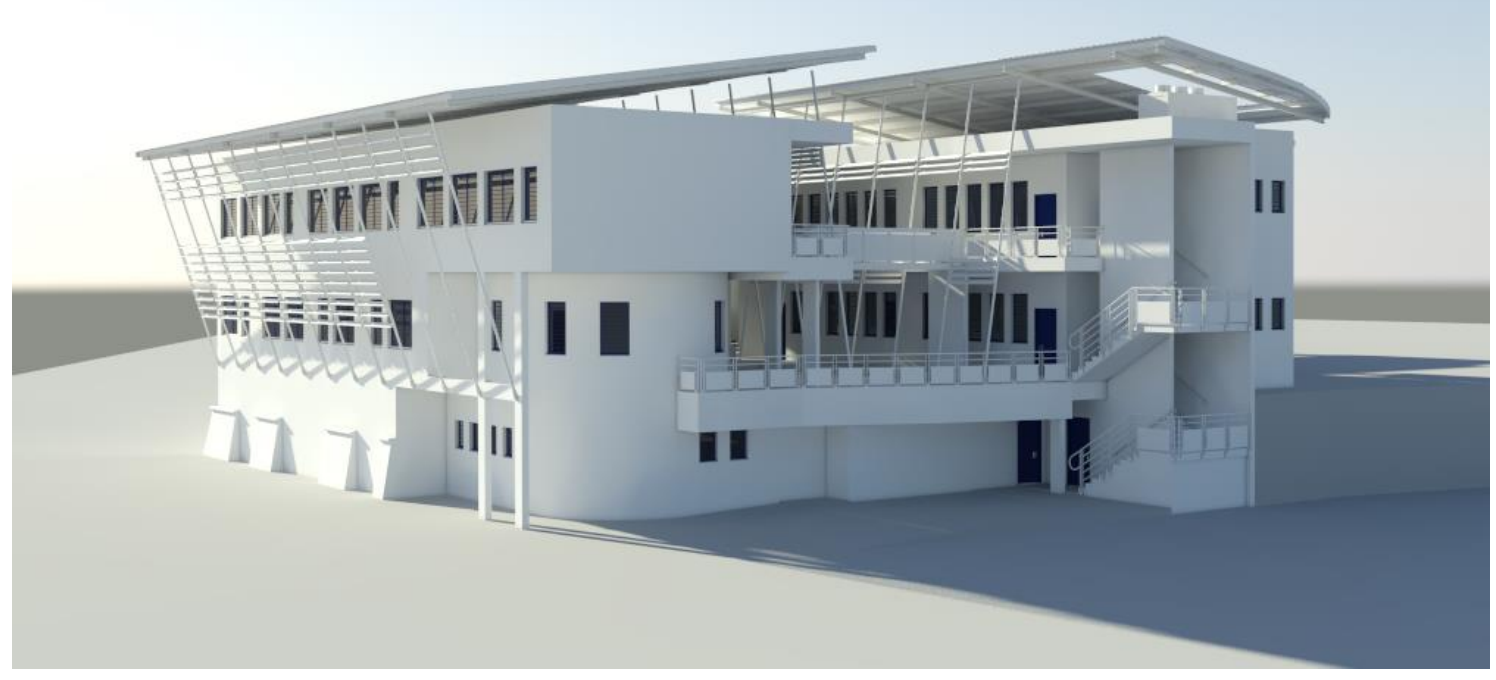

This model was then simulated, along with several lower resolution versions with increasing amounts of detail removed. This began with the simplification of the doors, window frames and mullions (figure 7 below, external shading removed for clarity) with the lack of mullions being accounted for with a $22 \%$ and $19 \%$ reduction in glazing visible light transmittance. These are proportional to the area of mullion on the North and South sides respectively. A second lower resolution model tested a reduction in detail of the shading devices directly outside the windows (figure 8 below): here the simplifications mainly consisted of the removal of the mounting brackets and reducing the polygon count of the supports. In the last simplified model all objects in the scene were roughly modelled as simple shapes (figure 9 below). Each model in this sequence had measured reflectances applied to as many surfaces as possible, although these are not necessarily pictured here for clarity. In simplified cases where two or more objects of different reflectances were merged, a weighted average of their material reflectances was used. 
Figure 7: LPBS model with original (left) and simplified (right) window and door geometry

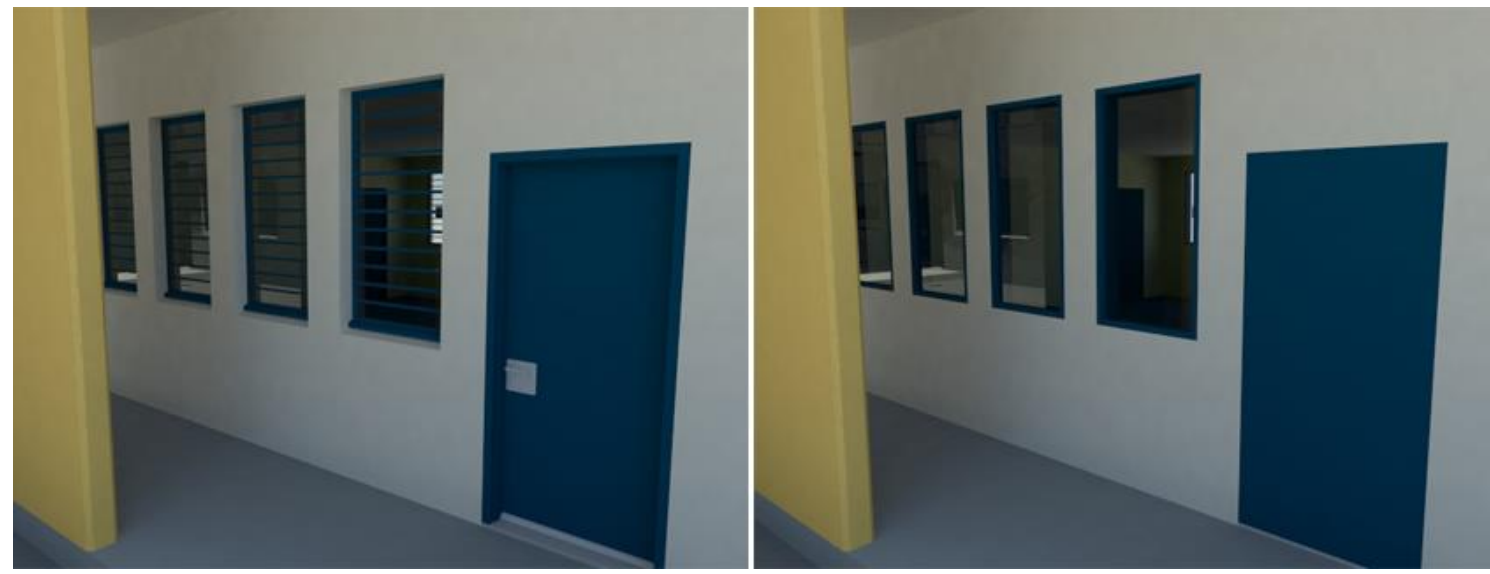

Figure 8: LPBS model with original (left) and simplified shading devices (right)
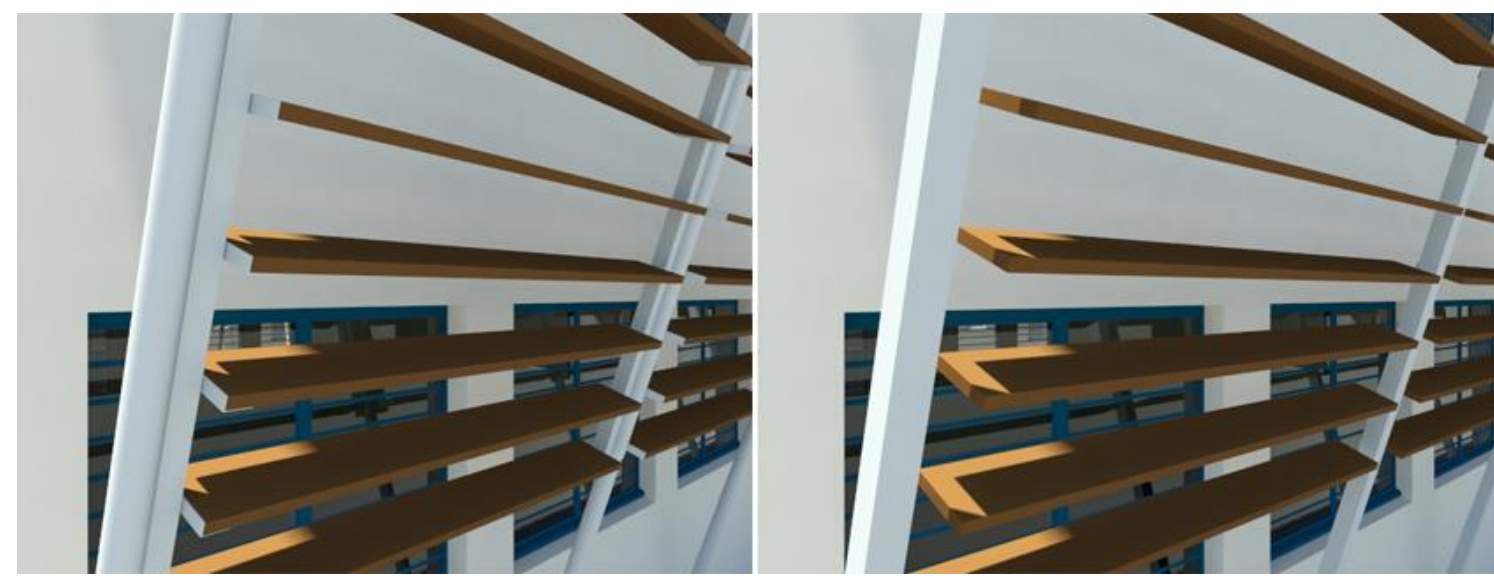

Figure 9: LPBS model with fully simplified geometry and without measured reflectances

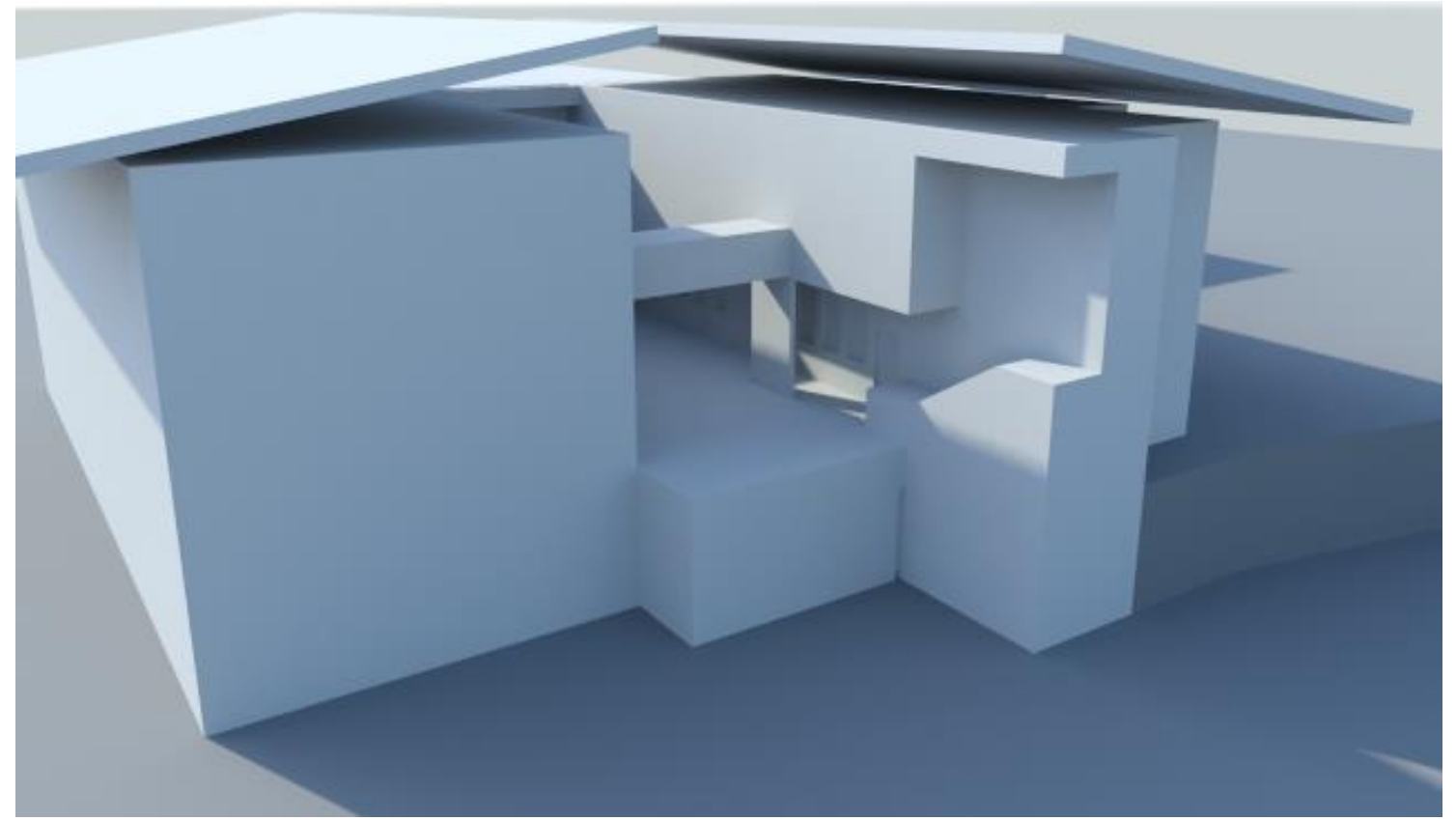


The tests on simplification of model geometry showed significant changes in predicted internal illuminances; however the magnitude of each was the opposite of what was expected. Simplifying the doors and windows showed an average increase in internal illuminances of $11 \%$, despite the mullions being accounted for in glazing transmittance. Mullions should therefore always be included in any daylight simulation model. The simplification of the external shading devices, in this case the timber louvers, showed a minimal change in internal illuminances. This was also unexpected. The decrease was by an average of $1 \%$, it is thought that this small change is due to this particular shading device already having fairly low complexity and therefore there was not a significant difference between the detailed and simplified geometry.

Simplifying the external geometry of the atrium and surrounding buildings had the greatest effect on internal illuminances. On average there was a 35\% increase in illuminance caused by the change, which can be likely attributed to the fact that the simplified geometry of the building across the atrium no longer contained deep concave sections, which were previously permanently shaded. This results in a significant increase of reflected light entering the atrium, and thereafter into the space being measured.

Together these tests show that model geometry simplification can have a considerable effect on the validity of a dataset. As a result; attempts should be made to create the most accurate simulation model possible; which requires a substantial number of measurements to be made unless complete, as-built construction drawings are available.

\subsection{Materials}

Similarly to the geometric dimensions of a space, there are often multiple sources for material data in a building design. These are occasionally measured, but more often come from manufacturers' data. The same risk that a model won't match reality applies; as materials are often assumed, simplified or change over time through fading or dirt accumulation. This section examines the sensitivity of daylight simulation models to the range of errors in measured reflectances and the effects of material simplification. It also assesses the sensitivity to changes in glazing according to precise manufacturers' glazing data, and examines the importance of the glazing maintenance factor.

\subsubsection{Measured reflectance data}

A test was devised for examining the potential error introduced by measurements of material properties. For each material in both of the empirically measured datasets, multiple measurements were made using a ColorMunki spectrophotometer (X-Rite Incorporated, 2008), and the results averaged to give the final material reflectance for the digital model. Typically, the variation between the highest and lowest values in each set of measurements was between one and $2 \%$, with a maximum outlier of $3 \%$. Thus, models were simulated with the averaged materials and the two "worst case" scenarios with materials of $\pm 3 \%$ reflectance values. It should however be noted that if illuminance measurements are being taken over a long period of time, such as a year, then reflectance measurements should be taken at several intervals during the measurement process to account for changes caused by material fading or dust collection if it is a problem.

Reflectance measurements were shown to have one of the largest effects on internal illuminances. For a $3 \%$ change in reflectances for all surfaces in the model, predicted internal illuminances rose by between one and $14 \%$, depending on the measurement point. On average for a $3 \%$ higher reflectance the space was predicted to be $6 \%$ brighter. The effect was again smallest at the measurement points nearest the window; which have a larger proportion of light coming either directly from the sky or via only one or two reflections. Measurement points near the back had the greatest change as they have a much larger proportion of light being reflected multiple times, compounding the error introduced by the change in reflectance.

It is imperative that considerable care is expended in ensuring the reflectances are measured with a calibrated device and for several points over the surface to check for changes across its area. 


\subsubsection{Simplification of material data}

The necessity for detailed reflectance measurements was tested by comparing models using different sets of reflectance data. The first model contained the measured reflectances for all surfaces. The second contained measured reflectances for just the surfaces inside the space being tested, with all other surfaces having a neutral near-white reflectance of 0.8 . The third model used typical "rule of thumb" reflectances for the interior, consisting of 0.3 for the floor, 0.5 for the walls and 0.8 for the ceiling, and again had a 0.8 reflectance for all other elements. The final model had a uniform white material with a reflectance of 0.8 . All models had an infinite ground plane with a reflectance of 0.2 . These represent steps further away from reality.

Following the reflectance tests above, it was likely that any simplification of model materials away from measured reflectances would have a substantial impact. When compared to a model with all surfaces containing measured reflectances, simulations using a model with only internal reflectances showed a difference of $20 \%$ in internal illuminances. Using common "rule of thumb" reflectances for the interior saw an even greater difference of $21 \%$ higher internal illuminances. Furthermore, a model containing uniform 0.8 reflectances showed an average illuminance increase of $46 \%$. This shows that measured reflectance data is essential to any daylight validation dataset, and that reflectances in general are the most critical element in any daylight simulation. This is likely due to the reflectance values having the most interactivity of any other aspect of the simulation, save for the simulation algorithms themselves.

\subsubsection{Glazing material properties}

Different glazing types from different manufacturers could be considered the finest high precision limit of input data in simulation that is a meaningful design choice. The effect of glazing type was tested using the LBNL test cell dataset. Two different glazing types were selected with a difference of only $2 \%$ normal Visible Light Transmission (VLT) between them. This was selected arbitrarily as the smallest VLT difference ever likely to be evaluated in a design study, and therefore the likely most precise a render would ever have to be. This resulted in a $4.7 \%$ difference in internal illuminances - a factor of 2.35 ratio between the VLT and the illuminance level.

This transmission change cannot be compared to the precision with which VLT is apparently measured: the International Glazing Database (LBNL, 2011) publishes VLT values to three significant figures, a $0.1 \%$ precision. This test revealed proportionally a much smaller change than a change in wall/floor/ceiling reflectance produces as discussed earlier.

A further test with a more drastic change of approximately $40 \%$ lower transmittance between clear glazing and a low VLT glass type led to a change in internal illuminances of $47 \%$. The ratio between change in VLT and change in average illuminance was 1.17. The lesson for the validation dataset is that errors in glazing transmission measurement will have a near-linear effect on the simulated light levels. Note that glazing with incidence-dependant coatings were not tested, as they are not compatible with 3ds Max Design or Radiance built in glass materials, instead requiring more advanced simulation techniques such as BSDFs not being tested as part of this study.

\subsubsection{Glazing transmittance: maintenance issues}

Manufacturers' data for glazing transmissivity is most often very precisely measured, and can thus be reliably input into a simulation model. However, as soon as the glazing is installed into a real-world situation, its transmittance will change due to the inevitable collection of dirt on exterior surfaces. Maintenance factors are often used in modelling to account for dirty glazing; and these factors can be as low as $70 \%(0.7)$ of the original transmittance for very dirty glass (Turner, 1969). Simulations were therefore conducted using glazing of the original manufacturers specification as well as with a VLT of 0.9 (10\% change) for clean glass and 0.7 ( $30 \%$ change) for very dirty maintenance factors. The goal was to determine the worst case errors that could be introduced.

Decreasing glazing transmittance is expected to have a significant effect on internal illuminance predictions, because all light entering the space must pass through the glazing. The trend observed with the VLT glazing transmission test above continued when the VLT changes of $10 \%$ and $30 \%$ were applied to take account of maintenance. Decreasing transmittance by $10 \%$ saw an $11 \%$ decrease in internal illuminance predictions. Decreasing the VLT by $30 \%$ saw an even greater reduction in illuminances by an average of $40 \%$. The patterns of illuminance reduction for all of the cases tested can be observed in figure 10 below, and are of a generally linear trend for normal VLT, and perfectly linear for hemispherical VLT. 


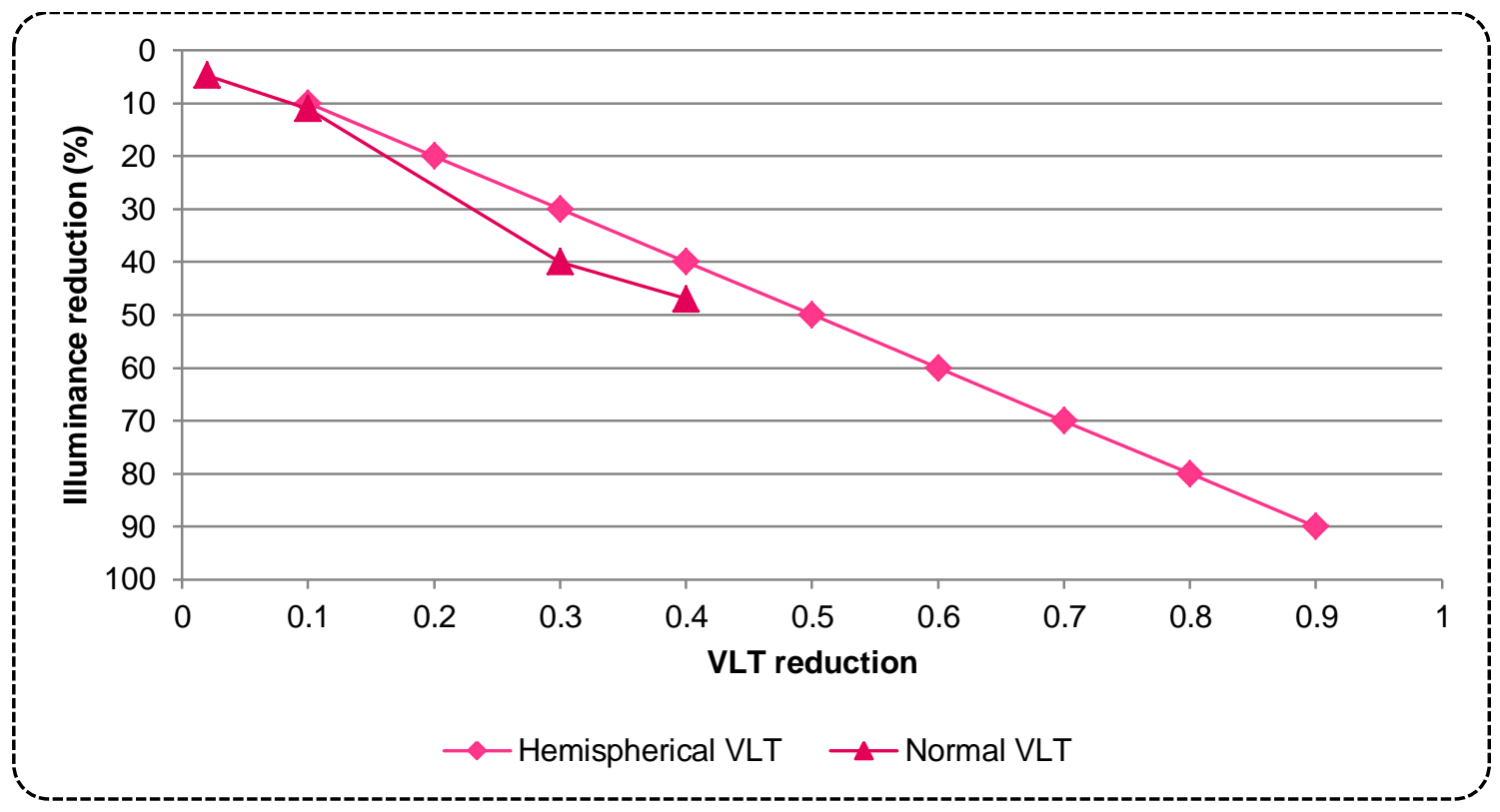

As the manufacturer's data for VLT is much more precise than the maintenance factor is, it is crucial that manufacturers' data is not entirely relied upon for glazing transmittance in a simulation validation dataset. The shading effects of dirt on glazing must be measured and assessed carefully.

\subsection{Sky models \& associated measured inputs}

As the sun and sky are generally the only light sources used in daylight simulations their accuracy is crucial to providing trustable results. Currently there are a number of different sky models available for use within daylight simulations; each with different benefits. Three common sky models are discussed below and their suitability for a validation study assessed. The models are the CIE general skies, as well as the Perez and Tregenza patch-based sky types. A newer, experimental fourth option, the use of High Dynamic Range Images (HDRI), is also considered. Following this, as this study makes use of the Perez sky for its empirical datasets, a number of tests have been performed to determine its potential effect on accuracy.

\section{CIE standard general skies}

The CIE standard or general sky set, containing both a clear and overcast sky representation amongst a number of other intermediate skies, have long been used with reasonable success for daylight factor calculations. However, in terms of validation, due to their being theoretical models rather than defined by real data; they are generally unable to be used in an empirical test scenario. Whilst a number of studies have shown that a mix of a statistically determined selection of skies from the set can be representational of the annual conditions of a location (Dumortier \& Kobav, 2007; Li, Lau, \& Lam, 2003; Shahriar \& Mohit, 2006), there is no set way to determine which skies to use for a typical year in an arbitrary location. This is required for any meaningful dynamic daylight simulation or annual daylight metric, such as the UDI. Conversely, their simplicity and rigidity makes them highly suitable for analytical validation work, such as within the CIE's own test cases, the results for which are discussed in the following chapter. 


\section{Perez sky model}

The Perez sky model is similar to the CIE models above in that it is simplistic; however it allows for a reasonable level of adaptability based on location, date and time, as well as measured data of the sun, sky and its turbidity via temperature and humidity. The model's simplicity, when compared to the following sky models, does give a large potential for error as it does not account for cloud positions in a partly cloudy sky. These discontinuous elements are instead lost in the diffuse contribution of the sky, smoothed across the entire hemisphere. The potential for error from this is significant, and is investigated in section 3.3.1 below. It is however proposed that this error contribution can be overcome through the use of a large number of data points over the course of a day, such as through the use of minute by minute data. This has the effect of smoothing out the error, and has been shown to give reliable results in previous validations such as Reinhart's 2009 validation of 3ds Max Design and Daysim. The Perez sky model is also currently the most advanced sky model that is widely available for use in daylight simulation software, and has a significant amount of data gathered worldwide that designers can use.

\section{Tregenza 145-patch and other patch-based skies}

The sky model first proposed by Tregenza is both the most expensive to measure and hardest to implement, but is also currently the most accurate tested sky description available to be used in daylight simulation. By dividing the hemisphere of the sky into a large number of patches, each individually measured by a sky scanner, a much higher resolution sky is produced. While there are some inaccuracies introduced by interpolation between the measurement points of the sky, these are far less than the hemispherical smoothing that occurs in the Perez and CIE models. Mardaljevic recommends that the Tregenza model become the "gold standard" for daylight validation studies (Mardaljevic, 2001; Mardaljevic, 2002). The sky scanner device required to make the measurements to produce the sky is a barrier to its use however; being the most expensive option available. This study is focused on building a dataset suite for daylight simulators that tests the software in the way it is likely to be used by designers. While over time the cost of sky scanning equipment will fall, without large collections of annual data for designers to use in their work its use will likely never become mainstream.

\section{High Dynamic Range Image skies}

While HDRI lighting has long been used in the field of architectural visualisation, it is still experimental in terms of physical accuracy for the use in daylight simulations. By combining digital photographs of the sky taken at many known exposures along with spot measurements of brightness; it is possible to generate a high dynamic range image of the sky with calibrated luminance. This can then be used to light a scene in a simulator; having many possible benefits. These include the possibility for a very high resolution sky far surpassing that of the Tregenza model above, as well as the ability to remove the need to model external obstructions as they can be "included" in the image. Further, the digital photos required to construct the images can be made with consumer-grade cameras, so long as their sensitivity is known, making them a relatively inexpensive method of data collection. A worldwide HDRI sky measurement database is therefore not unfeasible. This implies that HDRI skies will potentially become the most accurate and widespread method of creating sky descriptions in the near future. However, as with the sky-scanner measured patch-based skies, large collections of annual HDRI sky data do not exist and their use by designers will be minimal, hence they have therefore been excluded from this study.

\subsubsection{Discontinuous skies represented by continuous sky models}

As discussed above, there is the potential for significant differences between a real sky and a mathematically derived model of one, even if the model is based on measured data. This is due to the models' inability to reproduce the discontinuous nature of partly cloudy or intermediate skies. Some examples of this are shown below in figure 11 below, clockwise from top left: overcast, overcast-intermediate, clear, intermediate-clear; taken from Mardaljevic's $2000 \mathrm{PhD}$ thesis. Whilst previous studies such as Mardaljevic's thesis have examined this error contribution in real conditions, it is desirable to estimate what the maximum error that could be expected. 


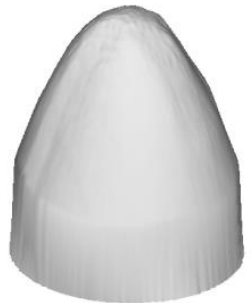

Lumscan

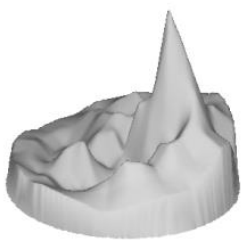

Lumscan

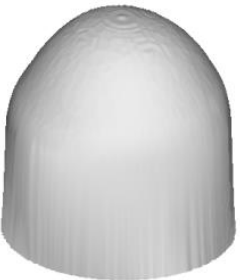

Perez model

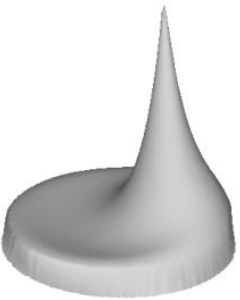

Perez model

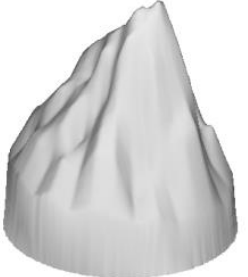

Lumscan

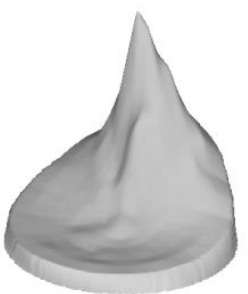

Lumscan

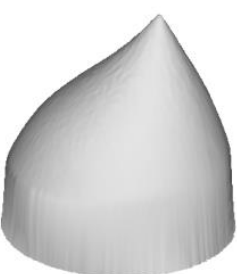

Perez model

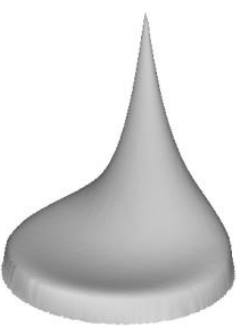

Perez model

A test was devised to test the maximum potential error contribution from an extremely discontinuous sky being modelled as continuous with the Perez sky model. This test compared the LBNL 71T test cell dataset's simulated internal illuminances under normal Perez sky model conditions to the same with an improvised 'cloud' in four different directions, but never covering the sun. An example of this is shown in figure 12 below, with the quarter hemisphere 'cloud' object in the South-Eastern portion of the sky above the model and ground plane. The cloud object's material is $100 \%$ diffusely translucent; meaning light hitting it is scattered equally in all directions with no absorption. On sunny days this creates a very bright area in one quarter of the sky.

\section{Figure 12: Quarter-hemisphere sky diffuser example}

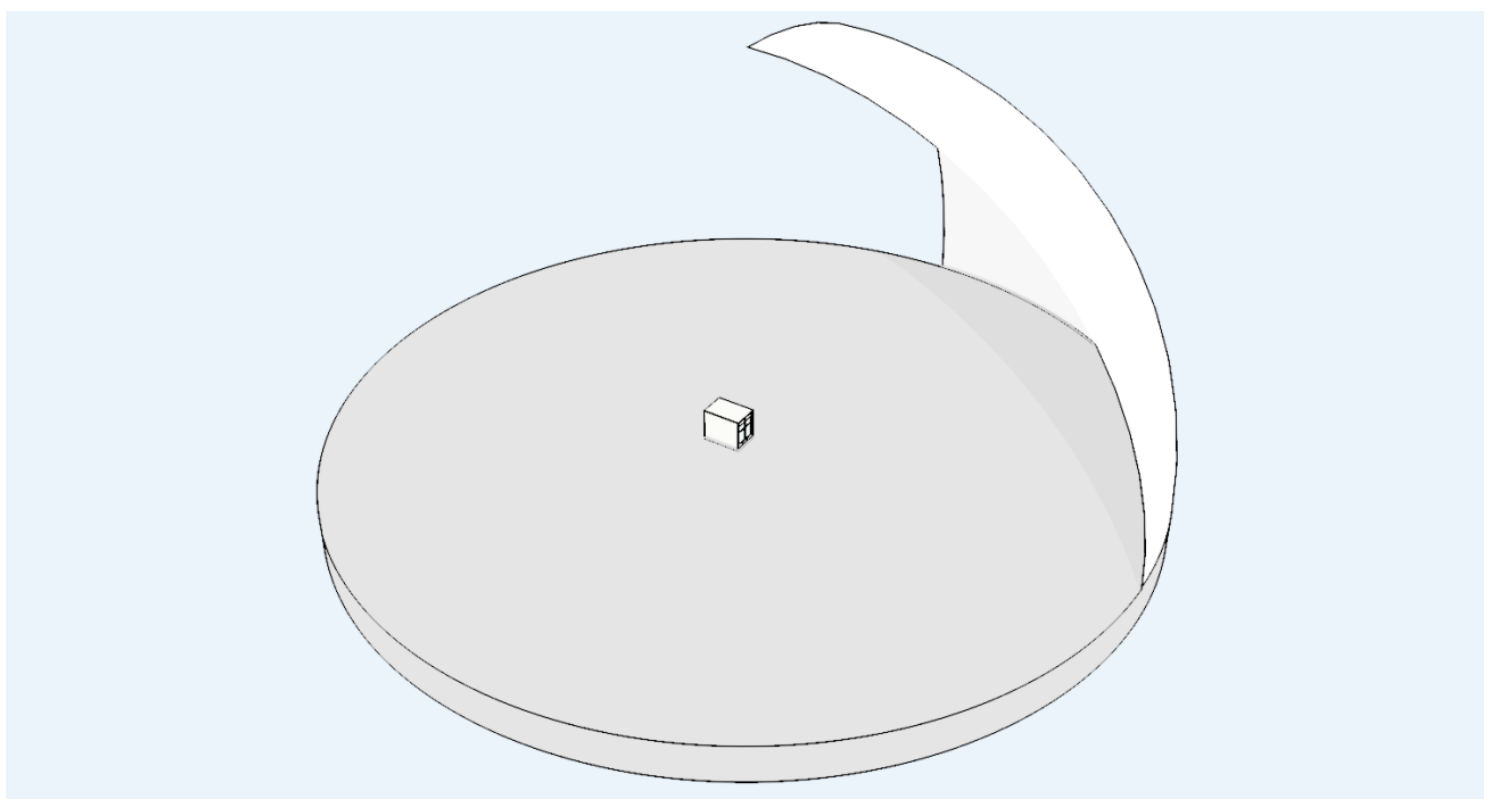

It was found that the least amount of change in internal illuminance occurred when the cloud object was in the North-Eastern quadrant, while the mid-afternoon sun was in the South-Eastern quadrant. The worst case scenario was found to be when the cloud object was in the South-Western quadrant with the sun again in the South-Eastern quadrant. With identical amounts of direct and diffuse light entering the scene, as much as a $37.5 \%$ increase in predicted internal illuminances was detected. This finding is of great significance, as it suggests that the error contribution from a simplified sky model such as the Perez model could be as high as $37.5 \%$ for a single data point; much greater than the contributions from poor reflectance data or errors in the model geometry. 


\subsubsection{Measured light and dew-point data}

The Perez sky model requires a number of inputs into its equations in order to produce its calculated sky luminances. In addition to the location, date and time, used for solar positioning, the most common inputs used by simulation software are direct and diffuse radiation for sky brightness, as well as dew point for sky haziness or turbidity (Perez, Seals, \& Michalsky, 1993). These values must be measured or estimated, and thus have a likelihood to introduce errors. The equipment used to measure radiation however is generally of very high quality; and is often rated to produce variations of only $\pm 1 \%$ or less; provided their operating temperature range is maintained (Eppley Labs, 2004). Tests were made with both the Berkeley and the Reunion datasets with the radiation data increased by $1 \%$, in order to determine the change in internal illuminances.

Because there is only one interaction with the sun and sky by a raytracing light simulation program - when a ray terminates at either of the light sources - the effect of any error in the sky brightness is directly passed on. For a $1 \%$ increase in the direct and diffuse radiation values input into the sky model, a $1 \%$ increase in internal illuminances was also recorded, and was the same for all measurement points. This shows that the effect of external solar radiation measurements is direct and that any error band for the sensors used to measure them can be translated to the internal illuminance measurements.

Dew-point is not directly measured; and is instead a product of temperature and relative humidity measurements. Further testing was conducted using $1 \%$ higher than measured temperatures and $5 \%$ higher relative humidities as a worst case scenario in dew-point measurement.

A worst-case difference of $1 \%$ increase in temperature and $5 \%$ increase in relative humidity can see the dew-point increase by a factor greater than the two individual errors combined. For the test conducted, there was an average increase in dew-point of $7 \%$. This in turn caused a $6 \%$ shift in the illuminances produced by the Perez sky model, and consequently a $6 \%$ shift in internal illuminances. This is of particular significance, as it is counter intuitive in daylighting validation datasets to focus on ensuring that temperature and humidity measurements are as carefully recorded as the daylight. It is apparent that they can have a greater influence on error than initial solar radiation measurements. Having synchronous and reliable measurements of temperature and relative humidity in conjunction with solar radiation data are therefore essential for any dataset intending to use the Perez sky model for simulation.

\subsection{Sensor point placement \& tilt tolerances}

The placement of light-meters within the space suffers from the same inaccuracies as initially measuring the space's dimensions, with an additional factor of the sensor's tilt. In order to determine the effect of the sensor's placement on the internal illuminances in a space, models were simulated with measurement points in the measured locations; as well as with the measurement points shifted $\pm 3 \%$ of the distance to the nearest surface in each of the principal axes.

A $3 \%$ error introduced into the positioning of light-meters into the spaces showed a maximum difference of $13 \%$ in predicted illuminance; with typically the largest difference being along the axis towards the space's openings. The size of this error was unexpected, as to the eye there was not a substantial visual difference in illuminance. The test specifically excluded directly-lit measurement points (sensors with direct line of sight to the sun), to try to avoid large changes in predicted internal illuminances at the edge of shadows. It is clearly extremely important that internal illuminance meters are placed as precisely as feasible.

Simulations were also run with the measurement point's normals tilted by one degree towards and away from the glazing in each space, considered the worst case conditions for alignment. A one degree tilt off level of the simulated measurement points with the measurement normal moving towards the glazing showed an increase in predicted illuminance of only $1.5 \%$. Tilting the normal by the same amount away from the glazing resulted in a decrease in predicted illuminance of $2 \%$. Errors introduced by likely inaccuracies in illuminance sensor angle are not as large as those introduced through inaccurate positioning. 
Not all data collected for a validation dataset will be viable for use; it is usually necessary to discard any obviously erroneous data. This may include where sensors have accidentally been disturbed by outside factors or where measurements go beyond a sensor's rated limits. However, it has also been recommended that any data that would not directly serve the validation purpose be discarded also. Mardaljevic advocates avoidance of internal illuminance data that includes a direct component; that is for when a sensor is in direct line of sight with the sun or circumsolar region.

The data entries that are likely to be affected by source visibility related errors are those where the (BRE office) photocell can 'see' the circumsolar region. The solution is to identify and exclude them from the validation. Note that this does not in any way weaken the validation. The direct component of illuminance is relatively trivial to compute. However, to compute it accurately (where the photocell 'sees' the circumsolar region) the luminance distribution around the sun must be known to an unattainable degree of accuracy. And the $3 D$ office model must be an exact representation of the actual office.

In fact, filtering biases the data to those instances where (difficult to compute) inter-reflection dominates. (Mardaljevic, 2002)

This is beneficial for a number of reasons; firstly being that computing the direct component of illuminance is relatively simple. This has the effect of biasing the validation towards more difficult inter-reflection calculations. Secondly; with the current collection of sky models it is very difficult to compute the luminance of the circumsolar region around the sun to a high enough accuracy for simulations where its component dominates. Lastly, direct sunlight casts shadows with sharp edges; and to model a space with enough detail to recreate the moving shadow boundaries over a day accurately is not practical.

Figure 13: Typical shadow movements over measurement point
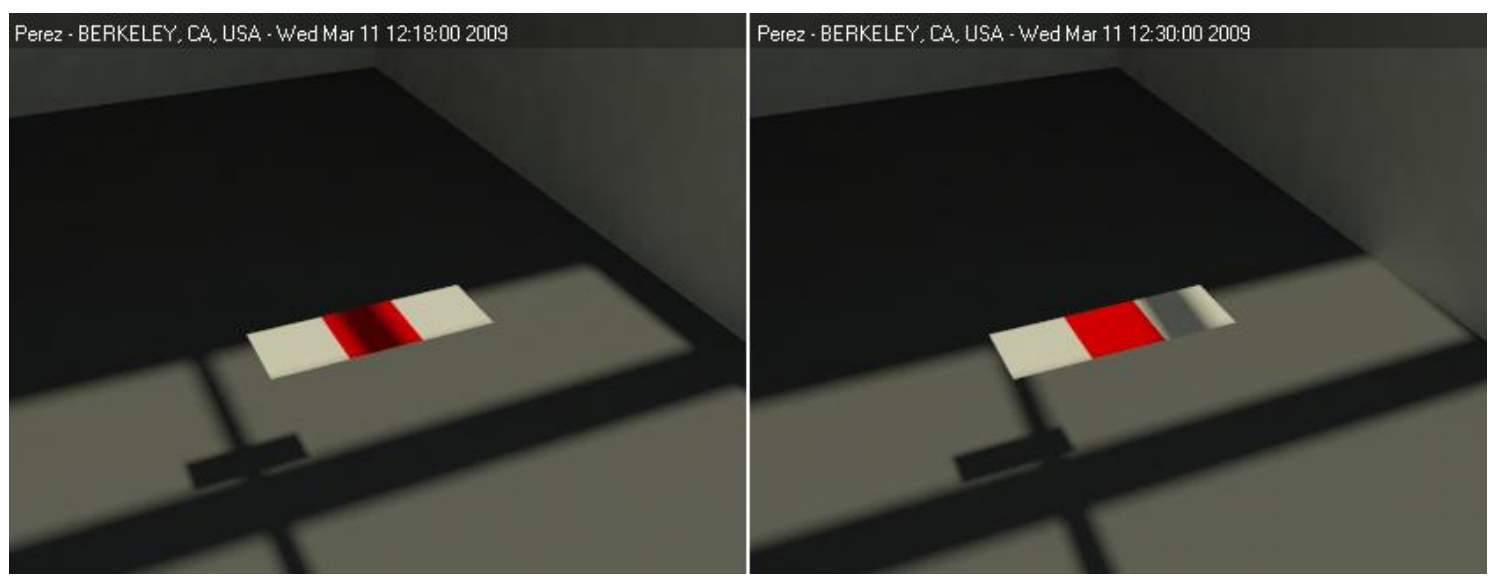

Figure 9 above shows the movement of a shadow cast by a mullion in a South-facing window in Berkeley, California, on a day near the equinox. The middle shaded part of the surface represents the area that would potentially be occupied by an internal illuminance sensor positioned one metre from the window with a $\pm 3 \%$ margin of error in its position. In order for the shadow band to move completely off the shaded area, 12 minutes must elapse. It can therefore be recommended that not only should data that contains direct illuminances be discarded; but all data 15 minutes either side of passing in or out of direct sunlight should also be discarded to decrease the likelihood of errors from slight inaccuracies in modelling. None of the measurement points for the LPBS dataset ever encounter direct sunlight.

It is also recommended that data from within half an hour of sunrise or sunset be discarded due to the previously documented inaccuracies of the Perez sky model for low sun angles where the local atmospheric and geographic conditions gain significance (Perez, Seals, \& Michalsky, 1993). Further, because at these times the direct and diffuse radiation or illuminance values used as inputs are quite small, small measurement errors can translate to large Mean Bias Errors or other accuracy metrics; potentially skewing the results towards inaccuracy. 
Each of the aspects of the datasets can, using the results above, be ranked in terms of importance based on their likely contribution to error. The rankings are as follows:

1. Model material detail.

2. Model external geometric detail.

3. Perez sky model simplification.

4. Internal reflectance measurements.

5. Model opening geometric detail.

6. Horizontal positioning of internal illuminance sensors.

7. Glazing transmittance measurements.

8. Dimensions of room.

9. External temperature and humidity data used to calculate dew-point for the Perez sky model.

10. Size and dimensions of glazed opening.

11. Rotational positioning of internal illuminance sensors.

12. Measurements of mullions or other simple obstructions.

13. Direct and diffuse solar radiation measurements.

These rankings reveal several conclusions. Firstly, that the most critical elements of any daylight simulation dataset are the reflectances, both internal and external. Without precise reflectance data for most surfaces in the simulation model it is unlikely a quality validation dataset can be achieved. Second, model detail is of unexpected importance, particularly for setbacks and shaded areas present in the complex LPBS dataset. Potential error from the continuous nature of the Perez sky model is again unexpectedly high; suggesting that there is a great need for more detailed sky models to become more prevalent in daylight simulation software, and suggests the possibility of using of many time-step data to average the errors out in the meantime. Positioning of internal sensors was also determined to have a large impact on the quality of a dataset. While ensuring their being level is not as crucial, precise measurements of their positions is highly recommended.

Perhaps most interestingly, measurements of direct and diffuse solar radiation ranked the lowest out of all aspects, and were determined to be much less critical than the dew-point measurements for the Perez sky, provided quality equipment is used.

\subsubsection{Lessons for designers}

In addition to these conclusions, a number of lessons can be gleaned for designers running their own daylight simulations. Whilst it may seem that the obvious method of increasing internal illuminances is to increase the size of the glazed opening, or to increase the glazing transmittance, these results show that it is potentially much more effective to increase internal reflectances or even shrink the room by a small amount to reduce its surface area. Critically, the results have shown that designers must provide a significant level of geometric and material detail to their models to even have a hope of achieving results with any indication of real world performance. Real reflectance values must be used, and any object with significant surface area, which is entirely dependent on the model, or that shades an adjacent building must be included. 


\subsubsection{Ideal properties of a validation dataset}

The ideal properties of a validation dataset using simplified sky models can then be listed:

- A large number of time-steps are required, hourly for a year or minute-by-minute for several days. This is to overcome the errors introduced by simplistic, continuous sky models.

- $\quad$ Must not contain times for which internal illuminances have a direct component, nor are within 15 minutes of having crossed from direct sunlight into purely indirect illumination.

- Must contain as geometrically detailed a model as possible, with precise reflectance and dimensional measurements.

- Extra care must be taken around openings, particularly with the overall opening area and the transmittance of glazing which must take into account the dirtiness of the glass. Mullions must be present but fine details are not required.

- Highly precise positioning of internal sensors is essential; however their levelness does not require the same precision.

- Highly accurate external temperature and humidity data is essential when using the Perez sky model, in addition to good direct and diffuse solar radiation data.

\subsection{Defining accuracy}

There are a number of different methods of measuring accuracy in daylight simulation validations, but the most common is the Mean Bias Error (MBE), followed by the Root Mean Square Error (RMSE). The MBE provides information on the long-term performance of the models, whereas the RMSE gives information on the short-term performance and indicates the amount of scattering of data around the models ( $\mathrm{Li}, \mathrm{Lau}, \&$ Lam, 2004). This study and its two empirical datasets will primarily make use of the Mean Bias Error, defined below in equation 1 , as it is the long term accuracy that is crucial for modern daylight metrics like Daylight Autonomy and UDI which use annual simulations. The CIE 171:2006 test cases however are generally of single results, and will have their accuracy measured using a more general error calculation on which MBE is based. This is defined below in equation 2: general error calculation.

$$
\begin{aligned}
& \text { Equation 1: Mean Bias Error (MBE) calculation } \\
& \qquad M B E=\left(\frac{1}{N}\right)\left[\sum\left(\frac{I_{\text {pred }}-I_{\text {mea }}}{I_{\text {mea }}}\right)\right]
\end{aligned}
$$

Where $I_{\text {mea }}$ is the measured internal daylight illuminance (lux); $I_{\text {pred }}$ is the predicted internal illuminance (lux); and $\mathrm{N}$ is the number of data points (dimensionless).

$$
\begin{aligned}
& \text { Equation 2: General error calculation } \\
& \qquad \text { Error }=\frac{I_{\text {pred }}-I_{\text {mea }}}{I_{\text {mea }}}
\end{aligned}
$$

Where again $I_{\text {mea }}$ is the measured internal daylight illuminance (lux) and $I_{\text {pred }}$ is the predicted internal illuminance (lux).

The CIE 171:2006 test cases have accuracy targets for some specific cases. As the test cases look at very specific aspects of light simulation with mathematically derived solutions, the cases without specific targets should match to the degree of rounding error within the simulation software; typically between $\pm 1-5 \%$. As these are highly case-specific, the accuracy target for each individual case is discussed in its relevant section in chapter 4.

It would be desirable to have an accuracy target of $\pm 5 \%$ for the two empirical datasets, as this is about the limit that the smallest likely design changes might be affected by program accuracy. However, without a more accurate sky description this is unreasonable. Instead; this study will use the widely accepted $\pm 20 \%$ value considered to be good accuracy in validations with mathematical sky models. As the largest change in illuminance in the tests above came to nearly $40 \%$, the datasets should aim to have none or very few data points outside of the $\pm 40 \%$ range. 

The CIE 171:2006 test cases to assess the accuracy of lighting computer programs are designed to test the multiple different aspects of light propagation in simulation software. This allows for the comparison of different programs with respect to these different aspects (CIE TC 3.33, 2005). This chapter documents the validation of both 3ds Max Design 2012 (version 14, service pack 2, educational license) and Radiance (version 3.9, "MinGW" release) against the test cases; with their results compared against both the analytical reference values and each other.

The validation using these test cases follows the digital modelling and simulation method prescribed in section 2.4, except where noted. For some cases additional simulation parameters have been trialled to test for improvements in simulation accuracy.

Table 3 on the following page lists all of the test cases assessed as part of this study; and whether both of the light simulation programs used for the study, 3ds Max Design and Radiance, achieved a 'pass' result. Nearly all of the cases from 5.3 onwards are important in relation to daylighting, barring the two symmetry cases in 6.10 and 6.11, and the mask to artificial light source case of 6.13. These daylighting-related cases have been highlighted in grey in the table. A number of points of interest were found for several cases, which have been numbered and described below the table. Short descriptions, including diagrams where required, of each of the test cases and the individual results for each are then listed under their own headings in the following sections. 


\begin{tabular}{|c|c|c|c|c|}
\hline Case \# & Title & $\begin{array}{l}\text { 3ds Max } \\
\text { Design pass? }\end{array}$ & $\begin{array}{l}\text { Radiance } \\
\text { pass? }\end{array}$ & $\begin{array}{l}\text { Results } \\
\text { Page \# }\end{array}$ \\
\hline 4.1 & Artificial lighting scenario - CFL, grey wall & Yes $^{1,2}$ & Yes $^{2}$ & 35 \\
\hline 4.2 & $\begin{array}{l}\text { Artificial lighting scenario - opal luminaire, } \\
\text { grey wall }\end{array}$ & Yes $^{1,2}$ & Yes $^{2}$ & 35 \\
\hline 4.3 & $\begin{array}{l}\text { Artificial lighting scenario - semi-specular } \\
\text { reflector luminaire, grey wall }\end{array}$ & Yes/No ${ }^{1,3}$ & Yes/No ${ }^{1,3}$ & 35 \\
\hline 4.4 & Artificial lighting scenario - CFL, black wall & Yes $^{1,2}$ & $\mathrm{Yes}^{2,3}$ & 38 \\
\hline 4.5 & $\begin{array}{l}\text { Artificial lighting scenario - opal luminaire, } \\
\text { black wall }\end{array}$ & Yes $^{1,2}$ & Yes $^{2}$ & 38 \\
\hline 4.6 & $\begin{array}{l}\text { Artificial lighting scenario - semi-specular } \\
\text { reflector luminaire, black wall }\end{array}$ & Yes/No ${ }^{1,3}$ & Yes/No ${ }^{1,3}$ & 38 \\
\hline 5.2 & Simulation of point light sources & Yes & Yes & 40 \\
\hline 5.3 & Simulation of area light sources & No & No & 42 \\
\hline 5.4 & Luminous flux conservation & $\mathrm{Yes}^{4}$ & $\mathrm{Yes}^{4}$ & 44 \\
\hline 5.5 & Directional transmittance of clear glass $\mathrm{T}$ & Yes & Yes & 45 \\
\hline 5.6 & Light reflection over diffuse surfaces & Yes & Yes $^{6}$ & 46 \\
\hline 5.7 & Diffuse reflection with internal obstructions & Yes $^{5}$ & Yes $^{5}$ & 49 \\
\hline 5.8 & $\begin{array}{l}\text { Internal reflected component calculation for } \\
\text { diffuse surfaces }\end{array}$ & Yes $^{6}$ & Yes $^{6}$ & 50 \\
\hline 5.9 & $\begin{array}{l}\text { Sky component for a roof unglazed opening } \\
\text { and the CIE general sky types }\end{array}$ & Yes $^{6}$ & Yes $^{6}$ & 52 \\
\hline 5.10 & Sky component under a roof glazed opening & $\mathrm{Yes}^{6}$ & Yes $^{6}$ & 52 \\
\hline 5.11 & $\begin{array}{l}\text { Sky component and external reflected } \\
\text { component for a façade unglazed opening }\end{array}$ & Yes $^{6}$ & Yes $^{6}$ & 54 \\
\hline 5.12 & $\begin{array}{l}\text { Sky component and external reflected } \\
\text { component for a façade glazed opening }\end{array}$ & Yes & Yes & 54 \\
\hline 5.13 & $\begin{array}{l}\text { Sky component and external reflected } \\
\text { component for a façade unglazed opening } \\
\text { with a continuous external horizontal mask }\end{array}$ & $\mathrm{No}^{7}$ & $\mathrm{No}^{7}$ & 56 \\
\hline 5.14 & $\begin{array}{l}\text { Sky component and external reflected } \\
\text { component for a façade unglazed opening } \\
\text { with a continuous external vertical mask }\end{array}$ & $\mathrm{No}^{7}$ & $\mathrm{No}^{7}$ & 56 \\
\hline 6.1 & Sun patches & Yes & Yes & 57 \\
\hline 6.2 & Specular reflections & No & Yes & 58 \\
\hline 6.3 & $\begin{array}{l}\text { Light transmission through ideal diffuse } \\
\text { glazing }\end{array}$ & Not tested $^{8}$ & Not tested $^{8}$ & 59 \\
\hline 6.4 & $\begin{array}{l}\text { Light transmission through bi-directional } \\
\text { glazing }\end{array}$ & Not tested ${ }^{8}$ & Not tested ${ }^{8}$ & 59 \\
\hline 6.5 & Light reflection on bi-directional surfaces & Not tested $^{8}$ & Not tested $^{8}$ & 59 \\
\hline 6.6 & Spectral calculation & Yes & Yes & 60 \\
\hline 6.7 & External illuminance variation & Yes & Yes & 61 \\
\hline 6.8 & $\begin{array}{l}\text { Daily and monthly variation of external } \\
\text { illuminance }\end{array}$ & Yes & Yes & 61 \\
\hline 6.9 & Light leaks into enclosed areas & Yes & Yes & 62 \\
\hline 6.10 & Room surface symmetry & Yes & Yes & 62 \\
\hline 6.11 & Light source symmetry & Yes & Yes & 63 \\
\hline 6.12 & Light source aiming & Yes & Yes & 63 \\
\hline 6.13 & $\begin{array}{l}\text { Internal shadows (mask to artificial light } \\
\text { source) }\end{array}$ & Yes & Yes $^{9}$ & 64 \\
\hline
\end{tabular}


1. The test case failed to achieve a pass using the supplied CIBSE photometric file format that is supplied with the test cases. This phenomenon is further described within the test case 4.1-4.3 section below.

2. The test case did achieve a pass using an IES photometric file format converted from the supplied CIBSE file format. Note that Radiance cannot accept the CIBSE file format, and thus required the inclusion of IES descriptions via its IES2RAD function. Further information is included within the test case 4.1-4.3 section below.

3. The test case, while achieving a pass for average illuminance, did not achieve a pass for all measurement points.

4. Test case requires an extremely large number of measurement points to pass.

5. CIE analytical values incorrect; pass achieved with recalculated analytical results.

6. Test case requires much higher than standard simulations settings to pass. These are described in the relevant test case sections below.

7. Test case shown to be invalid, see relevant test case section below.

8. Not tested due to undefined standard testing procedure.

9. Passed with anomalies present.

\subsection{Individual test case results}

\subsubsection{Test cases 4.1 - 4.3: Artificial lighting scenarios with diffuse inter-reflections}

\section{Test case description}

The first three test cases undertaken are all experimental rather than analytical in nature, and deal with electric light sources with measured photometric descriptions. Each of the cases makes use of a room with grey walls and four luminaires arranged evenly on the ceiling. Case 4.1 uses Compact Fluorescent (CFL) luminaires, 4.2 uses "Opal" luminaires, and 4.3 uses Semi-Specular Reflector (SSR) luminaires. Because of the grey or partially reflective walls in these cases, diffuse inter-reflections (being light not only directly from the source but also including light that has bounced off one or more surfaces) are taken into account.

Figure 14: Test case 4.1-4.6 room plan (CIE TC 3.33, 2005)

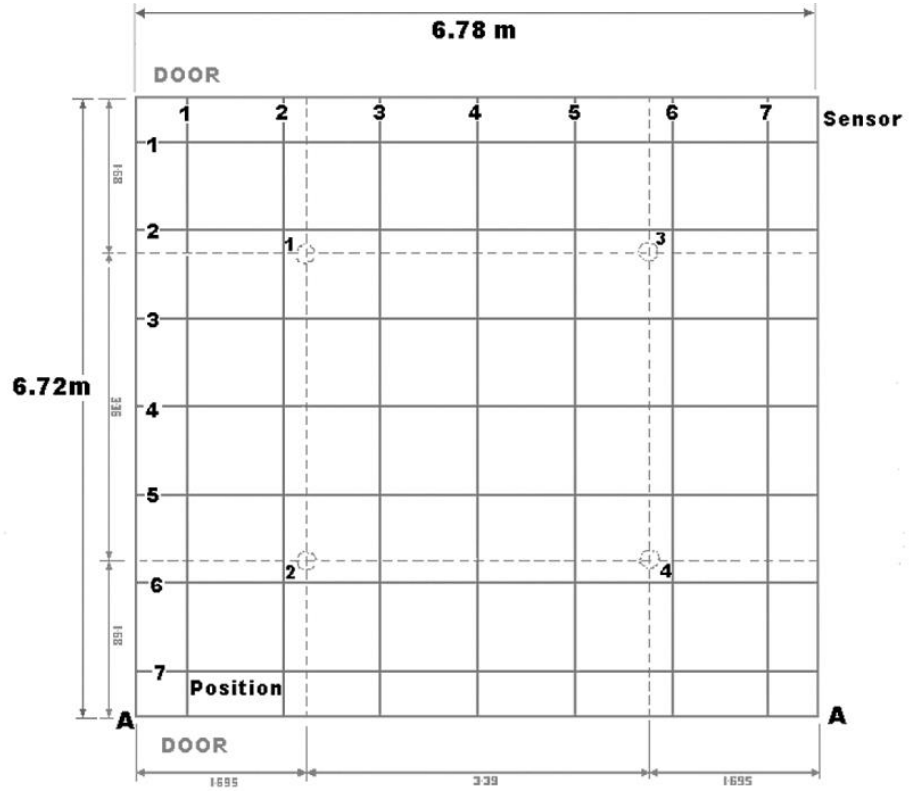

All three cases make use of a $6.78 \mathrm{~m}$ by $6.72 \mathrm{~m}$ room with a $3.24 \mathrm{~m}$ ceiling height; with a regular grid of four luminaires on the ceiling. All three cases have floor reflectances of $0.06 \pm 0.01$ and ceiling reflectances of $0.7 \pm 0.01$, with test case 4.1 having wall reflectances of $0.41 \pm 0.02$ and test cases 4.2 and 4.3 having wall reflectances of $0.52 \pm 0.02$. For the full test case descriptions refer to the CIE 171:2006 document. 


\section{Modelling issues}

Initial testing for these test cases showed significant error when using the CIBSE photometric file format within 3ds Max Design. For all cases 4.1 through 4.6, significant over-estimation of illuminances occurred, as can be seen for test case 4.1 in figure 15 below. Photometric files are text files that generally contain a description of the lamp; its manufacturer, type, size, shape, wattage; but critically a list of luminous intensities for an array or 'web' of angles around the luminaire. Two of the most common photometric file formats provide this list in different ways. The CIBSE file format (CIBSE, 1988) provides an array of candelas per 1000 lumens, whereas the IES LM-63-1995 format (IESNA, 2002) allows for the use of absolute or 'raw' candela values. It was noted that when applying the CIBSE photometric files supplied with the CIE 171:2006 document to light sources within 3ds Max Design, their photometric web was not displayed correctly. Upon examining the 3ds Max Design support documentation it was recommended that the more modern IES photometric description be used; with absolute intensity values.

Figure 15: Test case 4.1 Artificial lighting scenario; CFL, grey wall initial results

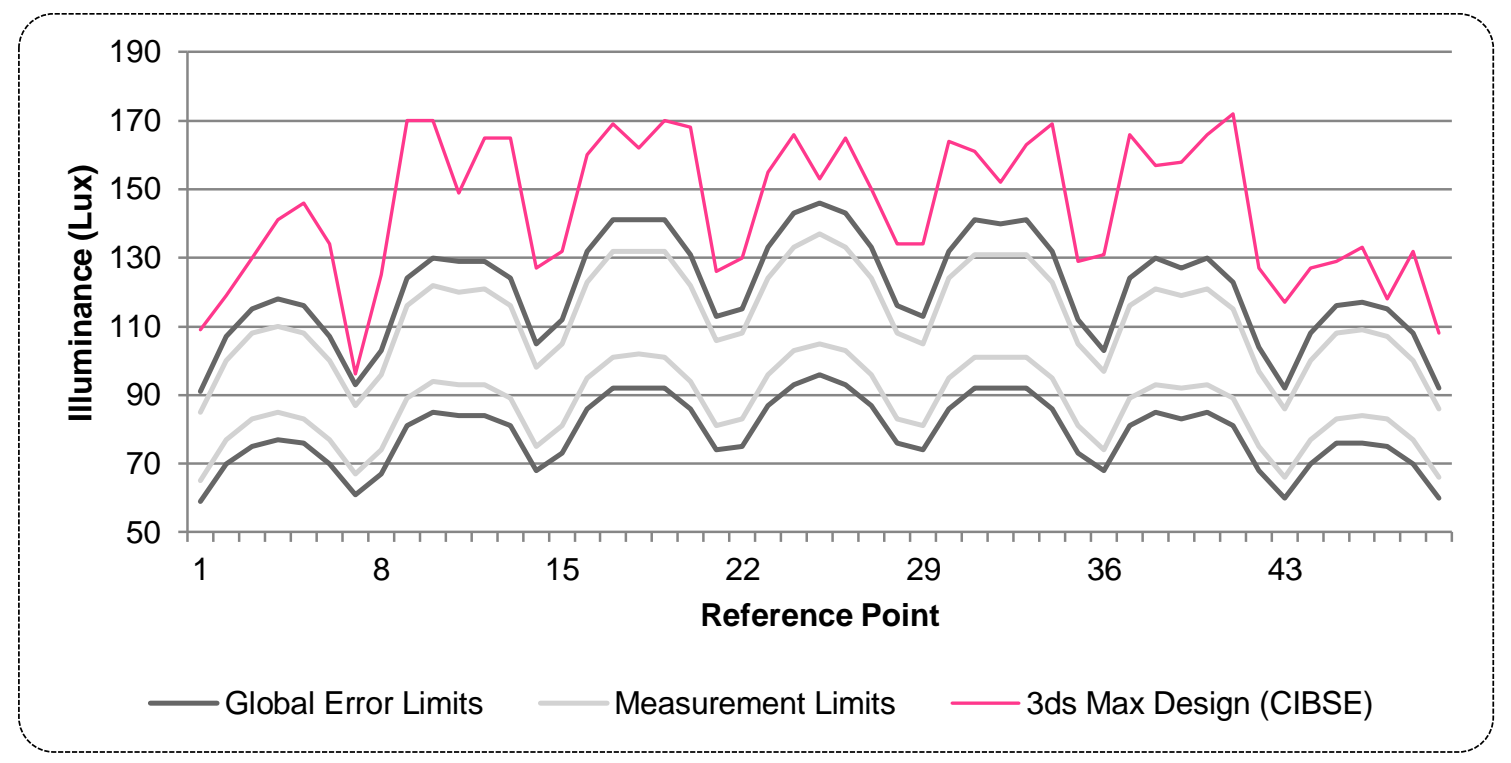

The supplied CIBSE file format photometric descriptions were converted to the IES LM-63-1995 file format, with absolute candela values and all multipliers set to one. Errors were significantly reduced when using the IES format with absolute photometry; without any added multiplication factors. It is therefore recommended that when using photometric descriptions for lighting in 3ds Max Design, the most recent IES photometry file format accepted by the software is used, with absolute photometry values. This is significant as 3ds Max Design, like many other lighting simulation software packages, is able to accept photometric files that can produce incorrect illuminance predictions for electric lighting. Further, users cannot tell when loading the IES file format whether it contains absolute photometry or not without opening the file in a text editor or other software. This means that there is a significant likelihood for electric lighting simulations to produce incorrect predictions through no fault of the user.

As a result of these findings, all of the test cases shown below have made use of the IES LM-63-1995 version of the format; with those files converted via IES2RAD for comparable use in Radiance.

\section{Simulation issues}

There were no simulation parameter issues with these three test cases.

\section{Results}

Figure 16 below shows one typical presentation format for displaying test case results. All 49 reference points for the seven by seven point grid of this particular case are displayed along the horizontal axis, with illuminances plotted on the vertical axis. The measurement limit plot is unique to the first six cases, 4.1 to 4.6, as they are based on empirically measured data, and shows the likely error range introduced by the measurement process. The global error limit plot shows the total between the measurement and simulation error limits, and the simulated illuminances must be within this range to achieve a pass for these cases. 


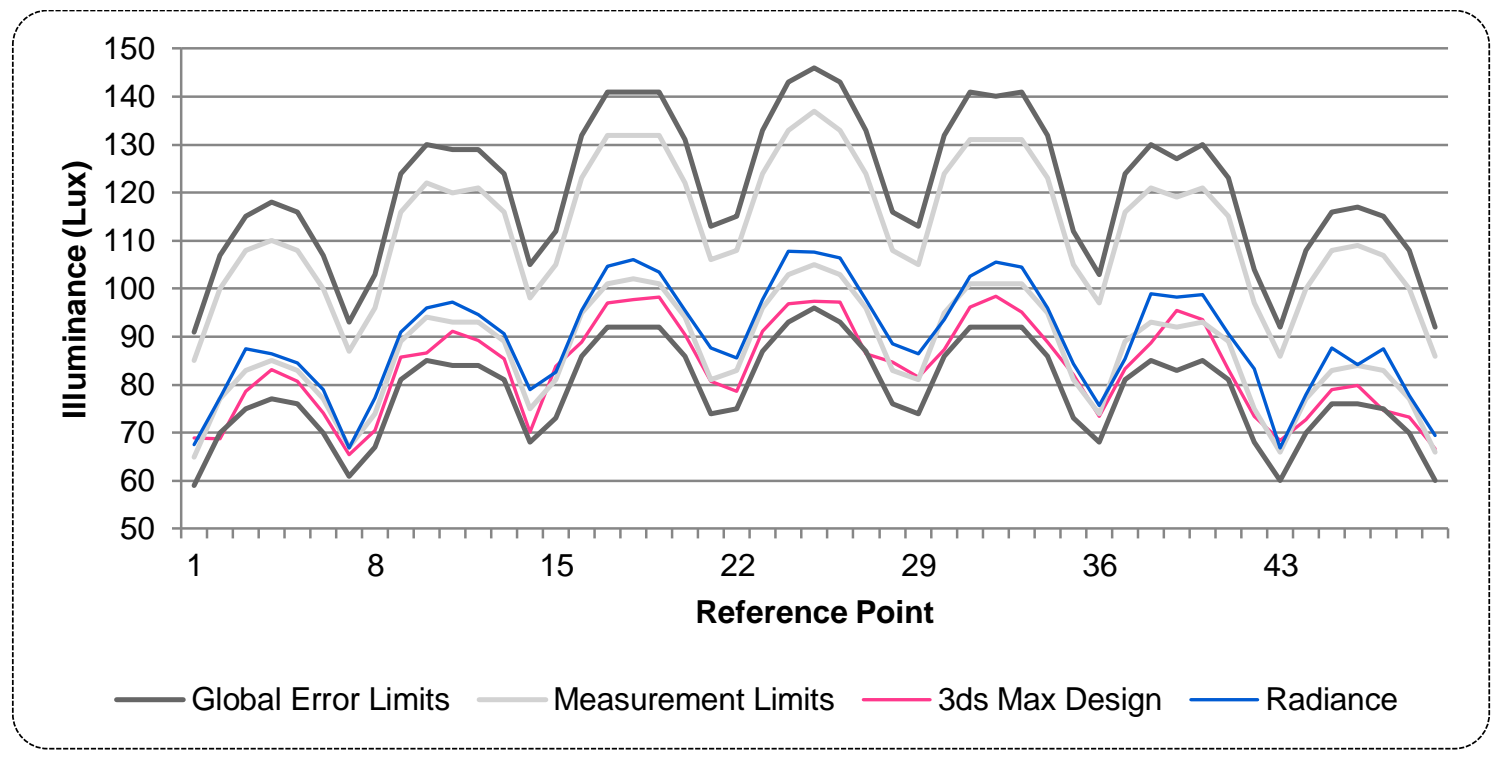

As figure 16 above shows, both 3ds Max Design and Radiance gave illuminance predictions for all points that were within the required global error limits for the CFL point light with grey wall scenario. Both simulators also showed room average illuminances within the global limits but with Radiance achieving an average closer to the centre of the range; however this was not a consistent trend as shown by the following test cases.

Figure 17: Test case 4.2: Artificial lighting scenario; Opal luminaire, grey wall results

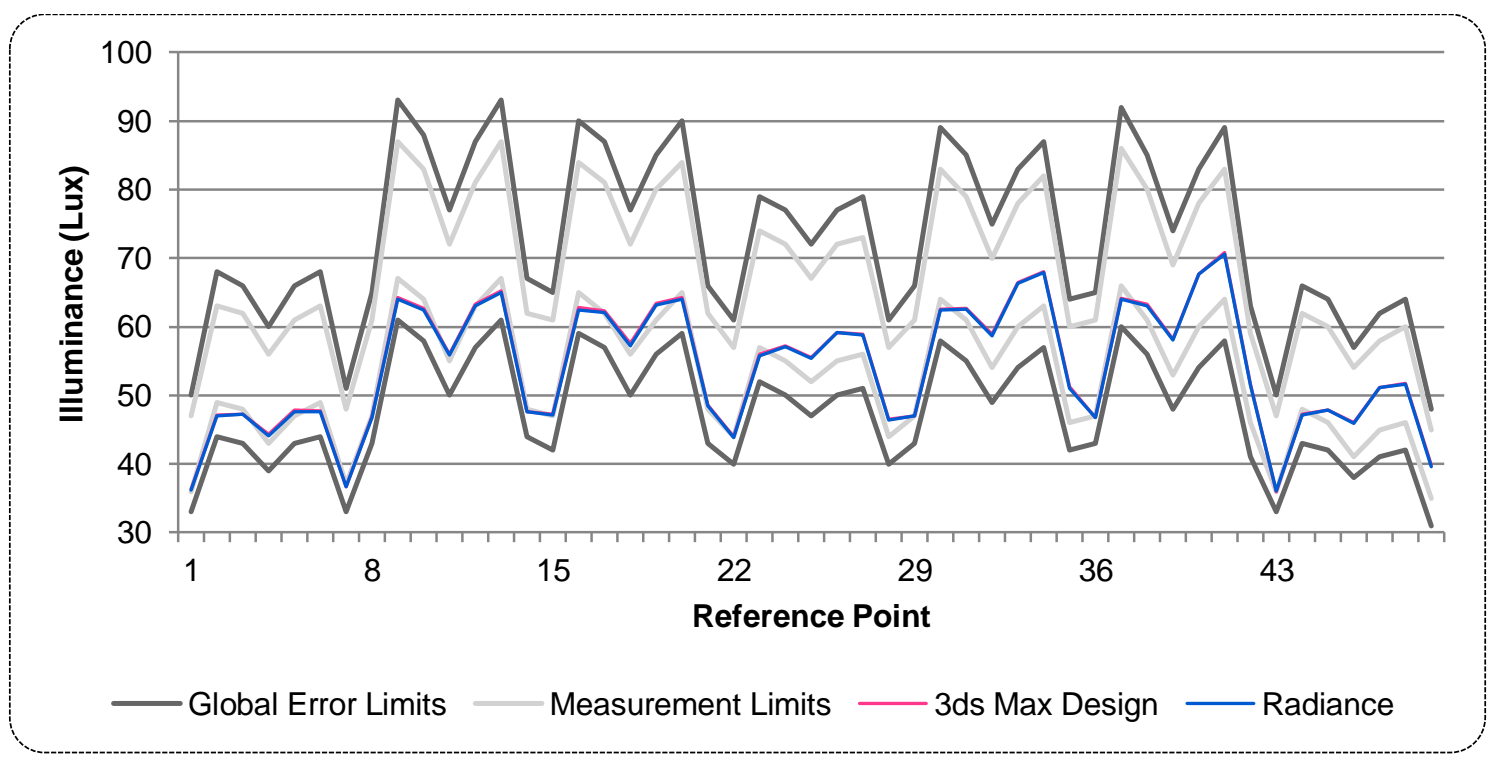

For the Opal luminaire point light with grey wall test case, figure 17 above again shows that both lighting simulators gave illuminance predictions within the defined global error limits. However, for this case both simulators produced very similar results with average room illuminances that are almost identical. 


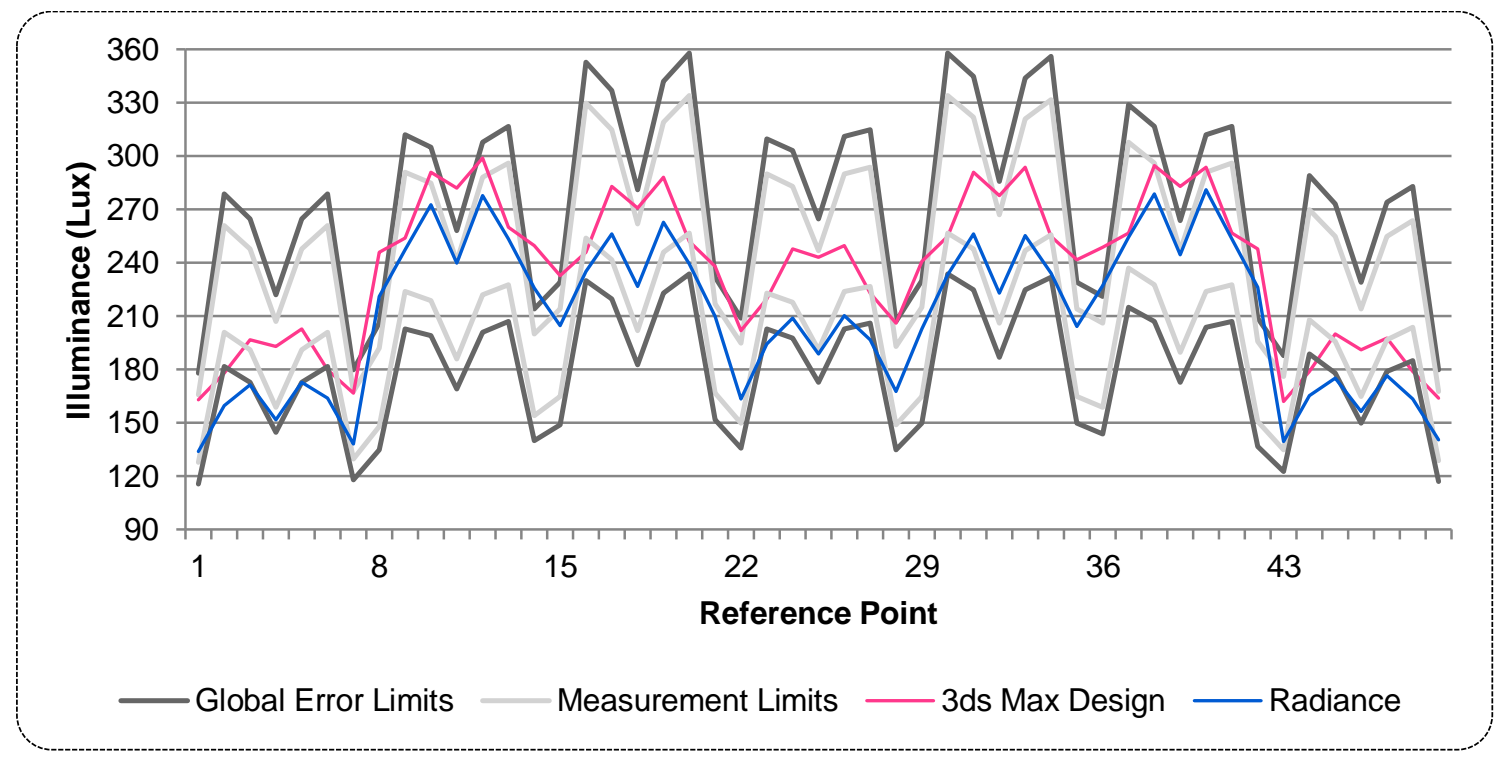

Test case 4.3 differs from the first two test cases in this section in that its photometric description is for a rectangular area light. The results shown above in figure 18 demonstrate that in this instance both $3 \mathrm{ds}$ Max Design and Radiance are not able to produce accurate illuminance predictions for photometric area lights. Illuminance predictions for both simulators fall outside the global error limits a number of reference points; however both simulators achieve average room illuminances within the global limits. A number of other test cases later in this suite also deal with area lights; and their results also show illuminances different to those predicted.

\subsubsection{Test cases 4.4 - 4.6: Artificial lighting scenarios without inter-reflections}

\section{Test case description}

Test cases 4.4, 4.5 and 4.6 are similar to 4.1, 4.2 and 4.3 respectively, making use of an identical room except with near-black surfaces to minimise the error introduced from inter-reflections. All three cases use ceilings with reflectance values of $0.03 \pm 0.01$, floor reflectances of $0.06 \pm 0.01$ and wall reflectances of $0.04 \pm 0.01$. Again for full test case descriptions refer to the CIE 171:2006 document.

\section{Modelling issues}

As these test cases make use of the same photometry as cases $4.1-4.3$, the same IES LM-63-1995 format photometric description files have been used for the relevant cases in place of the provided CIBSE format.

\section{Simulation issues}

There were no simulation parameter issues with these three test cases.

\section{Results}

As these cases are again based on measured data they follow the same result presentation criteria as cases 4.1-4.3 with all 49 reference points for the seven by seven point grid displayed along the horizontal axis, with illuminances plotted on the vertical axis. Again all simulated illuminances must fall within the global error limits. 


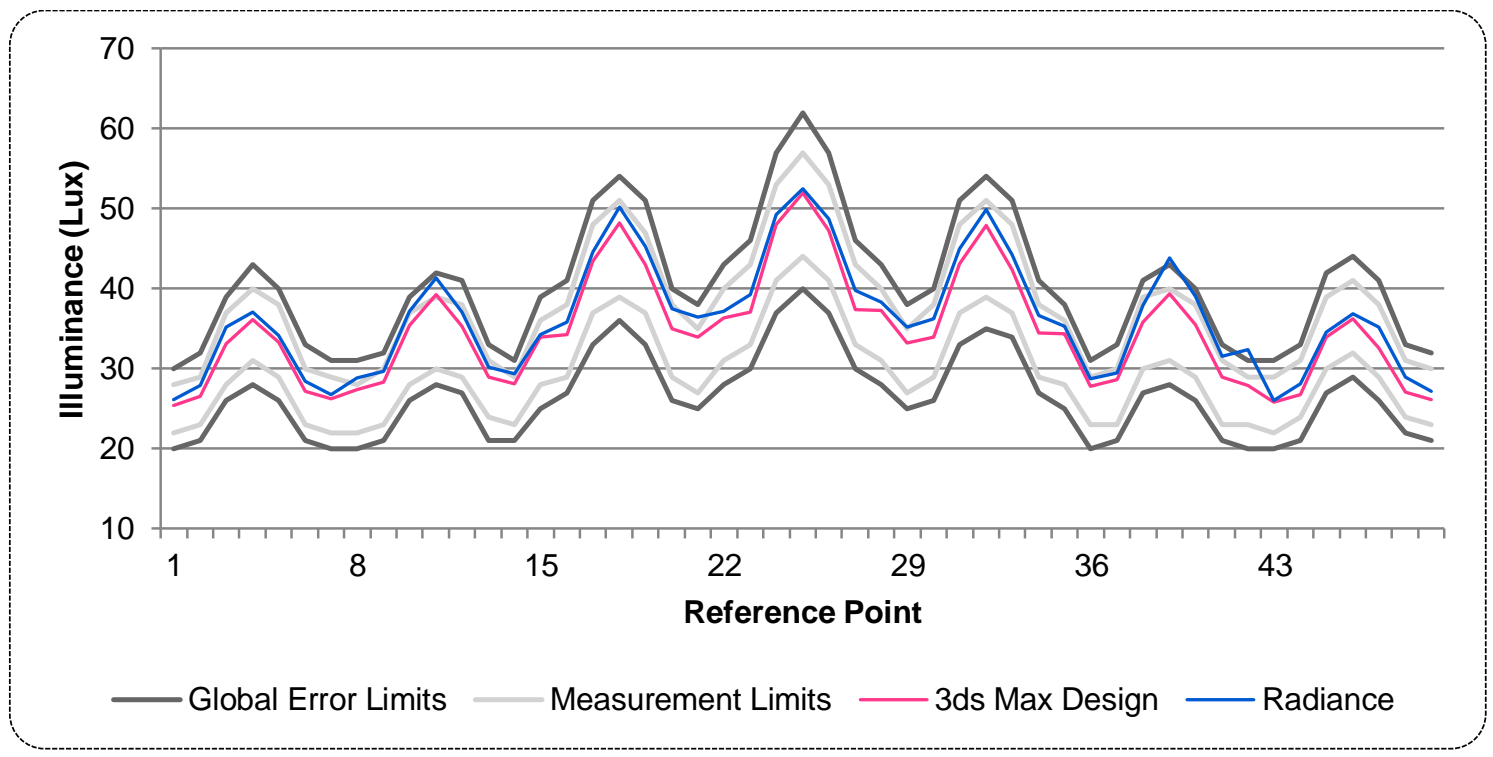

Figure 19 above shows that both 3ds Max Design and Radiance produced illuminance values that for the most part fell within the measurement limits and well within the global error limits of test case 4.4. Radiance produced two anomalous results for points 39 and 42, but overall the room average was within the tolerable limits outlined in the test case document. These spikes are likely a part of the normal "noise" in results produced by stochastic raytracing methods.

Figure 20: Test case 4.5: Artificial lighting scenario; Opal luminaire, black wall results

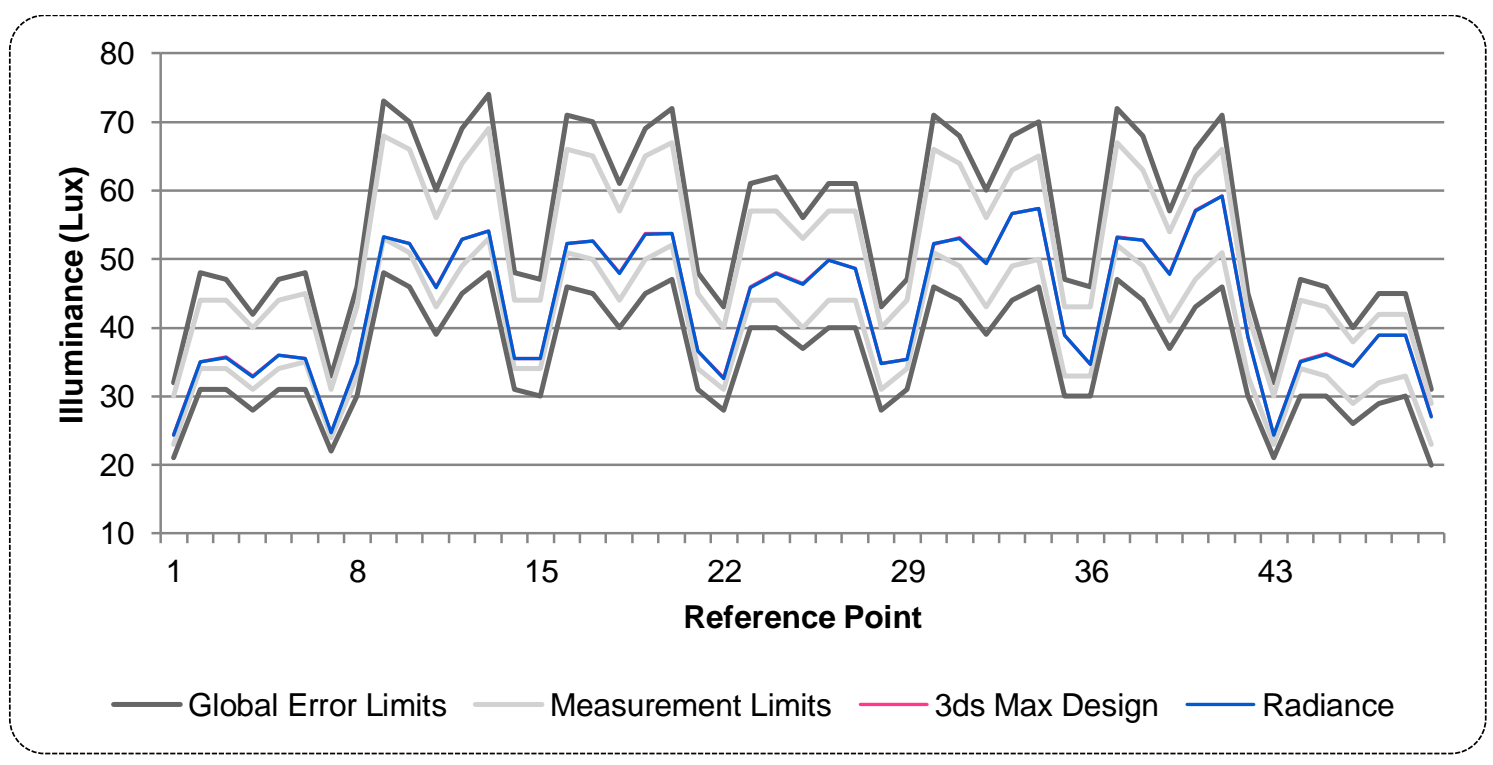

3ds Max Design and Radiance both produced very similar results, with their traces in figure 20 above overlapping for most points. Again the room average illuminances were within the target range. Together with cases 4.1, 4.2 and 4.4, these cases show that both simulators are producing accurate illuminance calculations for point lights with photometric descriptions, provided they are in the IES LM-63-1995 format. 


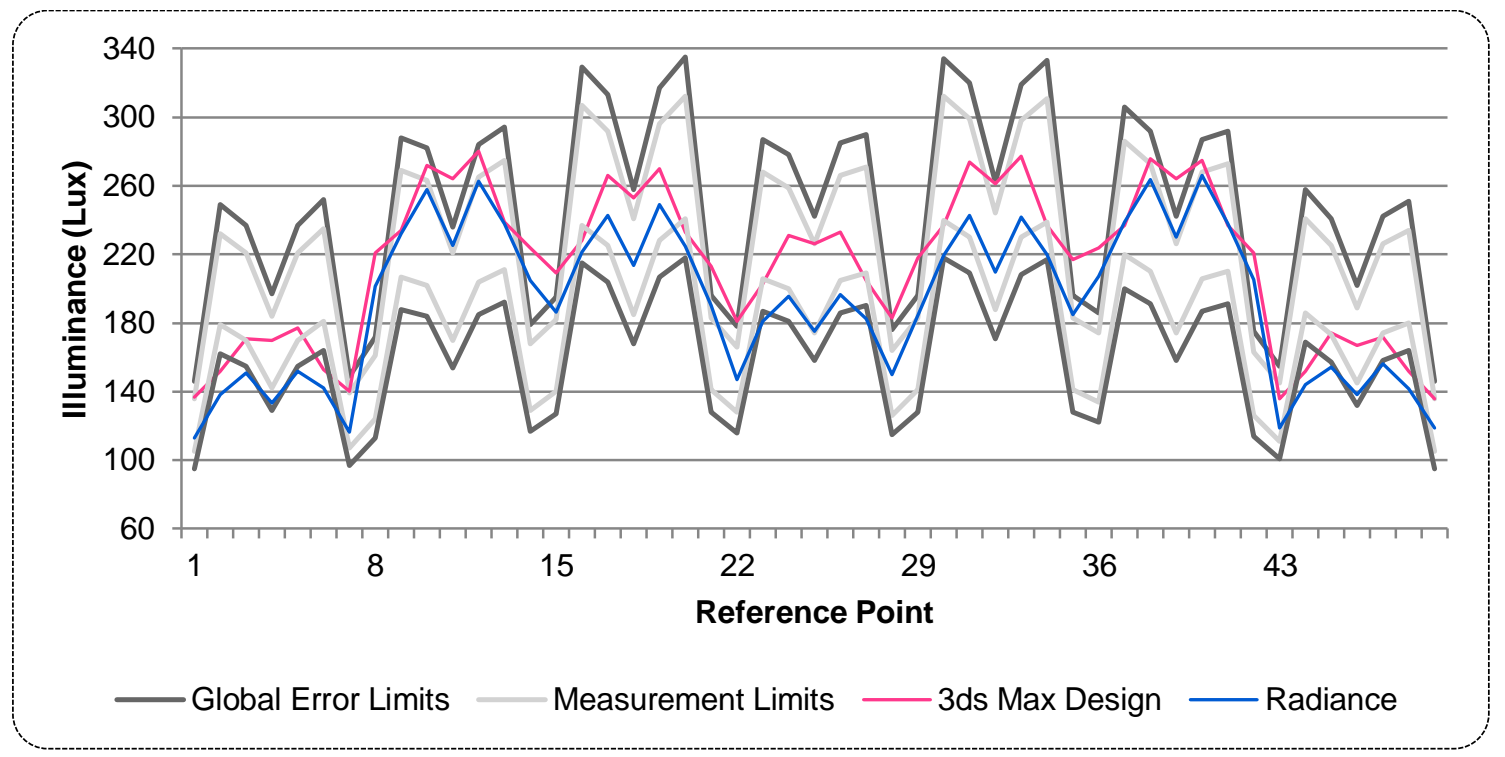

The results for test case 4.6 in figure 21 above show similar results to those of test case 4.3; also with the semi-specular reflector luminaire but taking into account diffuse reflections. With the diffuse reflections removed, it can be seen that the illuminance predictions for both simulations are still not meeting the global error limits for all reference points. This suggests that the errors are stemming from the area light sources in the simulations. Again, the room average illuminance was within the allowed limit, suggesting the amount of luminous flux entering the space is correct, but is not being distributed correctly.

The results of this test case in conjunction with those of test case 4.3 suggest there is a problem with the area lights in both 3ds Max Design and Radiance, where they are not distributing light correctly. This is further examined in test case 5.3 which also deals with area lights with analytical rather than measured references later in this chapter.

\subsubsection{Test case 5.2: Simulation of point sources}

\section{Test case description}

Test case 5.2 is the first analytically-based assessment in the suite; and tests both uniform diffuse and photometrically defined point light sources. The case is in two parts, both with the same geometry and measurement points, pictured in figure 22 below. Two different light sources, one uniformly diffuse (casting light uniformly in all directions) and one with photometry, shine on to a four metre by four metre square $100 \%$ absorptive surface. The light sources are positioned three metres directly above measurement point $A$ at the centre of the receiving surface.

Figure 22: Test case 5.2 measurement point distribution (CIE TC 3.33, 2005)

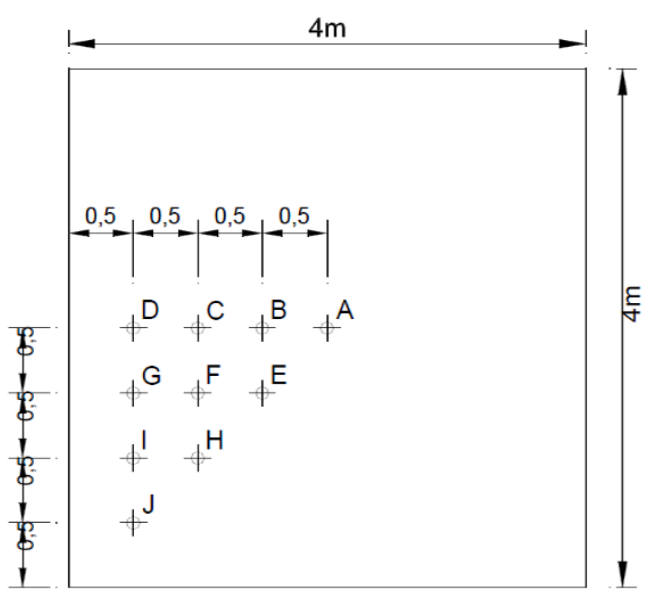




\section{Modelling issues}

This test case makes use of a photometric file, provided with the test cases in the CIBSE format. Due to the previous findings of cases 4.1 through 4.6, the file was first converted to the IES LM-63-1995 format with absolute luminance values for use in $3 d$ s Max Design and Radiance.

\section{Simulation issues}

There were no simulation parameter issues with this test case.

\section{Results}

Figure 23 below plots the analytically derived illuminances for each reference point in grey, with the simulated results of $3 d$ s Max Design and Radiance plotted in pink and blue respectively. This is the most common results format for the remaining analytical test cases; with the reference points on the horizontal axis and illuminances or other calculated value on the vertical axis.

Figure 23: Test case 5.2: Simulation of uniform diffuse point sources results

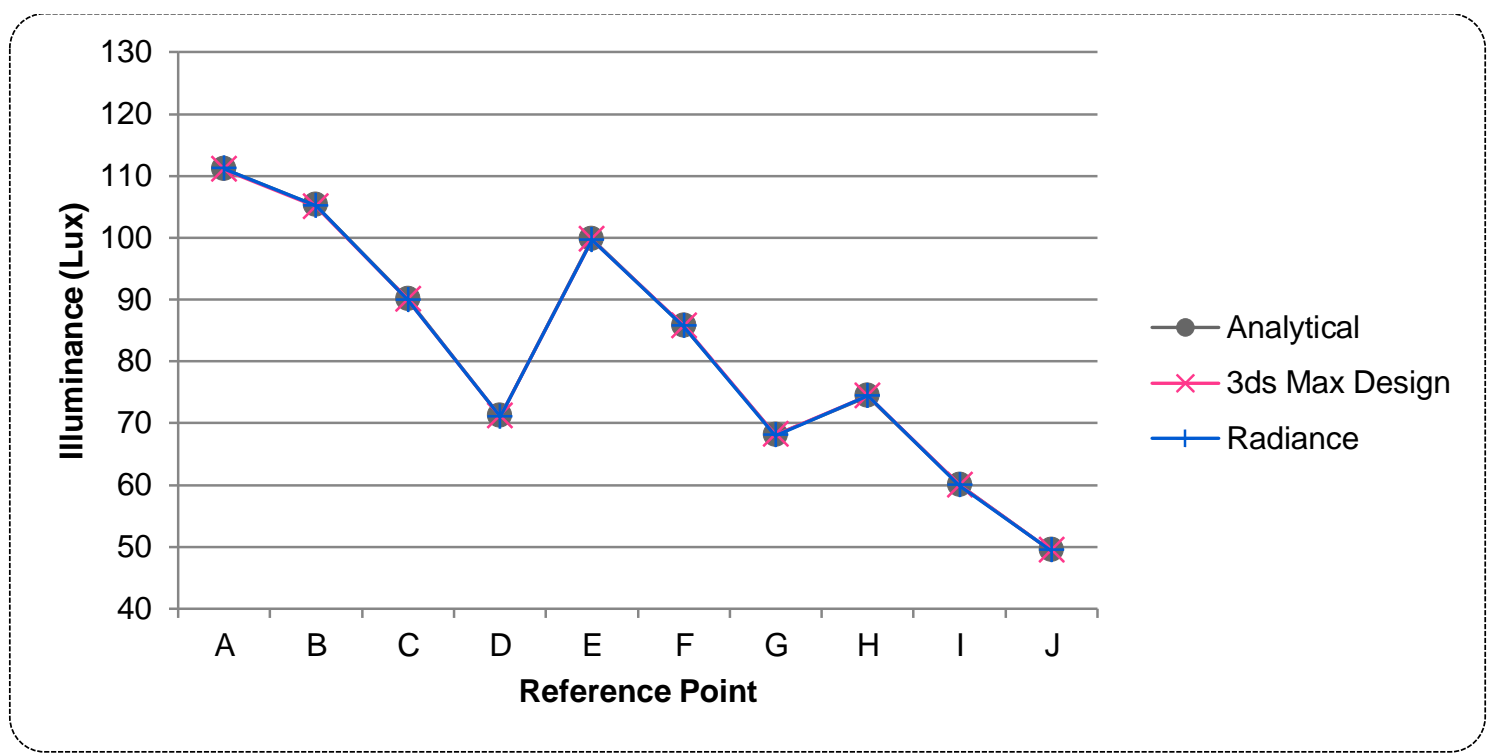

Figure 24: Test case 5.2: Simulation of photometric point sources results

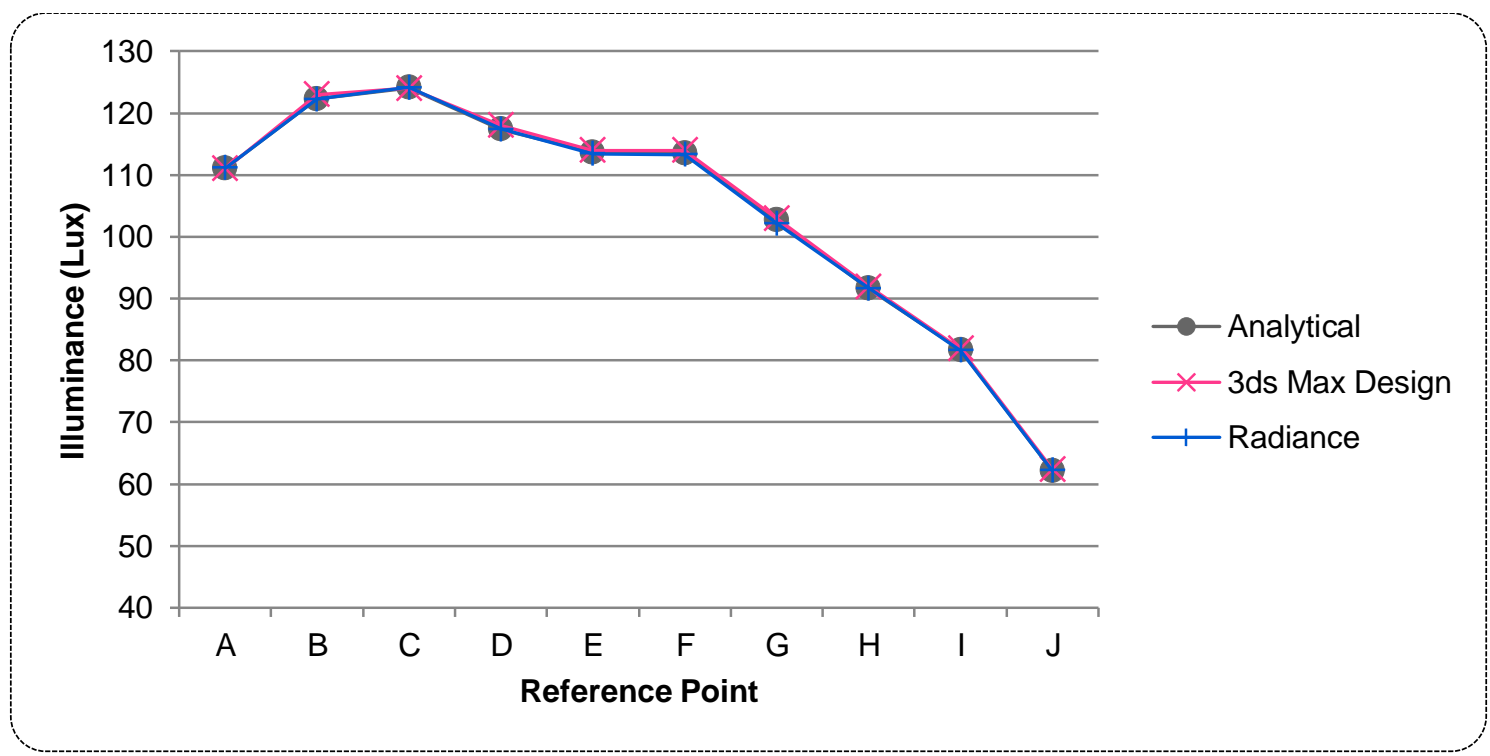

The results shown in figure 23 and figure 24 above re-confirm the accuracy of both simulators for illuminance predictions of point light sources shown in the earlier measurement-based test cases. For point light sources with and without photometry 3ds Max Design and Radiance produced illuminance predictions to within rounding error of the analytical reference values. 


\section{Test case description}

Test case 5.3 is intended to test the ability of simulation software's ability to produce illuminance predictions under area light sources. The test case consists of a four by four metre square room, with a three metre high ceiling. A one metre square area light is situated at the centre of the ceiling, with the measurement points positioned linearly across the centre of the room and up one wall, as pictured in figure 25 below. The test case looks both at a uniformly diffuse light distribution, as well as one defined by photometry.

\section{Figure 25: Test case 5.3 geometry and measurement points (CIE TC 3.33, 2005)}

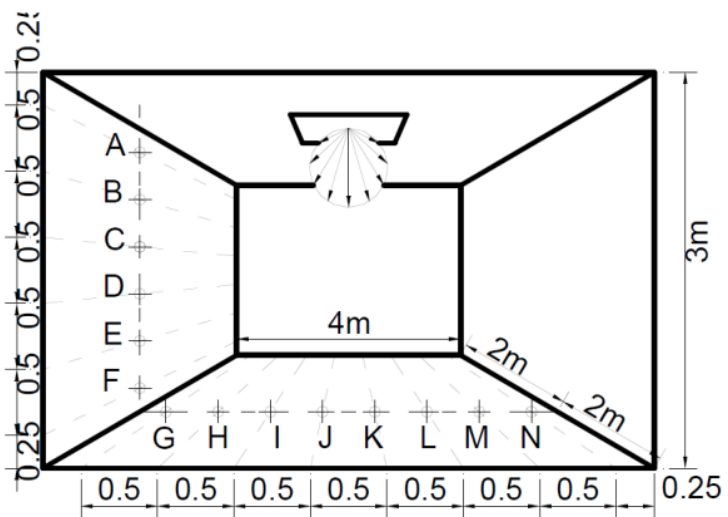

\section{Modelling issues}

This test case makes use of a photometric file, provided with the test cases in the CIBSE format. Due to the previous findings of cases 4.1 through 4.6, the file was first converted to the IES LM-63-1995 format with absolute luminance values for use in $3 d s$ Max Design and Radiance.

\section{Simulation issues}

There were no simulation parameter issues with this test case.

\section{Results}

Figure 26 below shows the results of test case 5.3 using a uniform diffuse area light source. It can be seen that for all reference points $3 d$ s Max design over-estimated the illuminances by varying degrees.

Radiance's illuminance predictions were much closer to the analytical results, but still outside what could be expected from rounding error.

As an additional experiment, a common technique in architectural and product visualisation within 3ds Max Design to improve the look of area lights was trialled, to test if it was an improvement over the software's default area light sources. The technique involves replacing the area light with a 'Daylight Portal' object, with a black body shader applied to it defining its brightness and colour temperature (Patton, 2007). This technique, plotted in purple, brought 3ds Max Design's illuminance predictions in line with Radiance, but was again outside the tolerable accuracy threshold. It is important to note that the majority of users would not likely use this technique as it is relatively unknown, and would more likely rely on the less accurate default area lights. 
Figure 26: Test case 5.3: Simulation of diffuse area light sources results

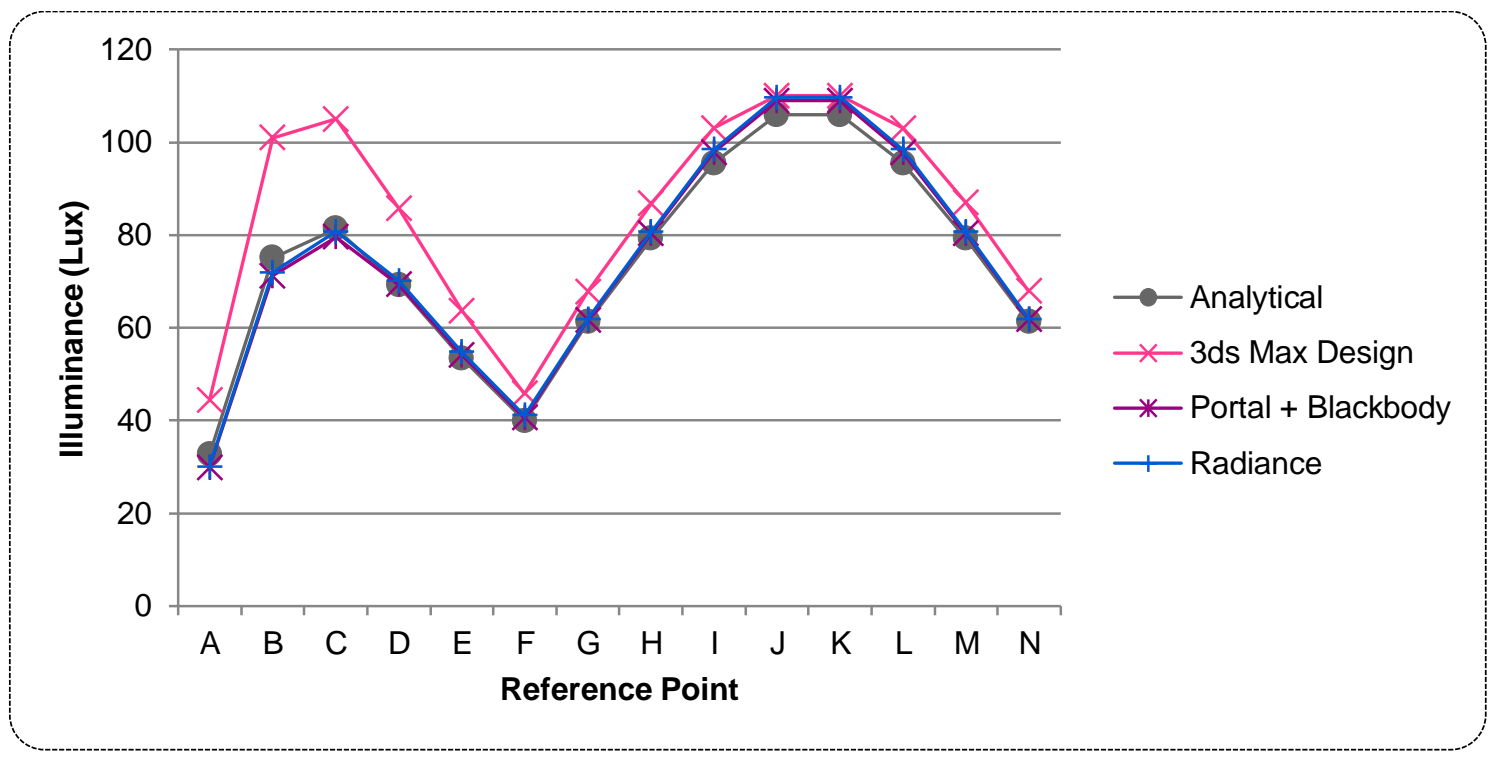

Figure 27: Test case 5.3: Simulation of photometric area light sources results

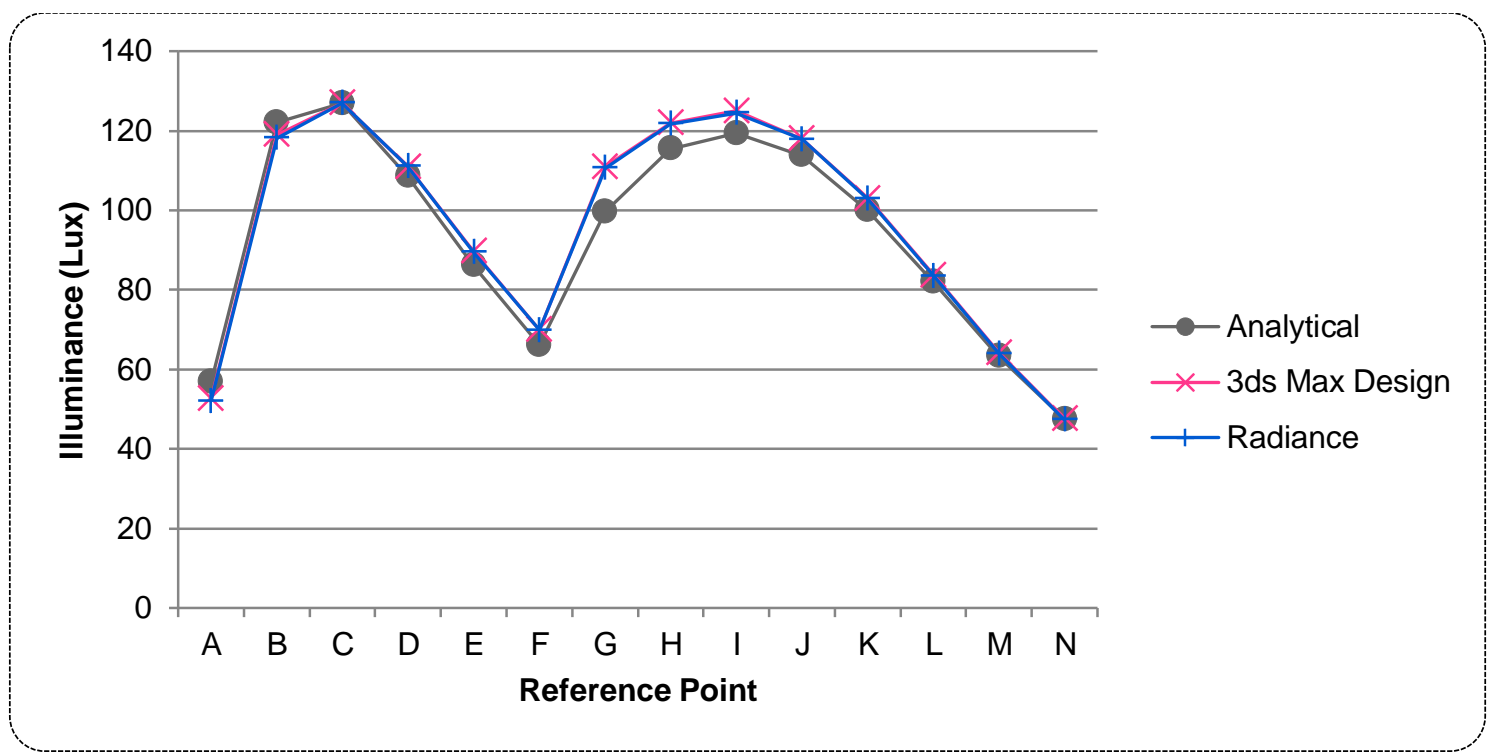

The results for the photometrically defined area light sources, shown in figure 27 above, show 3ds Max Design and Radiance's results closely agreeing, but are again well outside the rounding error accuracy limit. While initially this pointed to a potential error in the analytically calculated reference values, discussion with the development team for 3ds Max Design revealed that there are known issues with its calculations of light distribution from area light sources. It can therefore be assumed that the same issues are present in Radiance as well.

It can therefore be suggested that the developers of both simulators examine their software's techniques for simulating area light sources, as they have failed to produce accurate predictions in all cases within the suite that use them. It is also recommend that designers avoid the use of area light sources in simulations where the distribution of light is critical. 


\subsection{5}

\section{Test case description}

Test case 5.4 is intended to assess the luminous flux conservation between the light source and the internal surfaces of the space. The CIE 171:2006 test case document outlined several different geometric configurations for this case, but for this validation it was chosen to again use a four by four metre square room with a three metre high ceiling. A two by two metre square opening is positioned in the centre of the ceiling, modelled with a $200 \mathrm{~mm}$ thickness, and all surfaces have a $0 \%$ reflectance. A CIE clear sky was used as the light source, with a sun altitude of 60 degrees. A grid of ten by ten measurement points was placed over the opening to record incident flux, and similarly numbered grids over each of the receiving surfaces within the room to record received light.

\section{Modelling issues}

There were no issues related to modelling with this test case.

\section{Simulation issues}

This analysis of this test case originally used a grid of ten by ten measurement points on each of the receiving surfaces within the room; however this produced significantly erroneous results. Grids of points were required as 3ds Max Design does not natively provide a method to determine the total amount of light incident on a surface. In order to 'catch' a higher proportion of the light on the receiving surfaces, increasing numbers of measurement points needed to be used. The results for several numbers of measurement points are presented in the test case results below.

\section{Results}

Figure 28 below plots the number of measurement points in the grids on the receiving surfaces along the horizontal axis versus the recorded loss in luminous flux on the vertical axis. It can be seen that as the number of measurement points increases, the loss in luminous flux tends towards zero for both simulators. This suggests that both $3 d s$ Max Design and Radiance are conserving luminous flux in their calculations. Testing beyond a 100 by 100 grid on each surface was not done, as with ten receiving surfaces already having 10,000 measurement points, simulation times became prohibitive.

Figure 28: Test case 5.4: Luminous flux conservation results

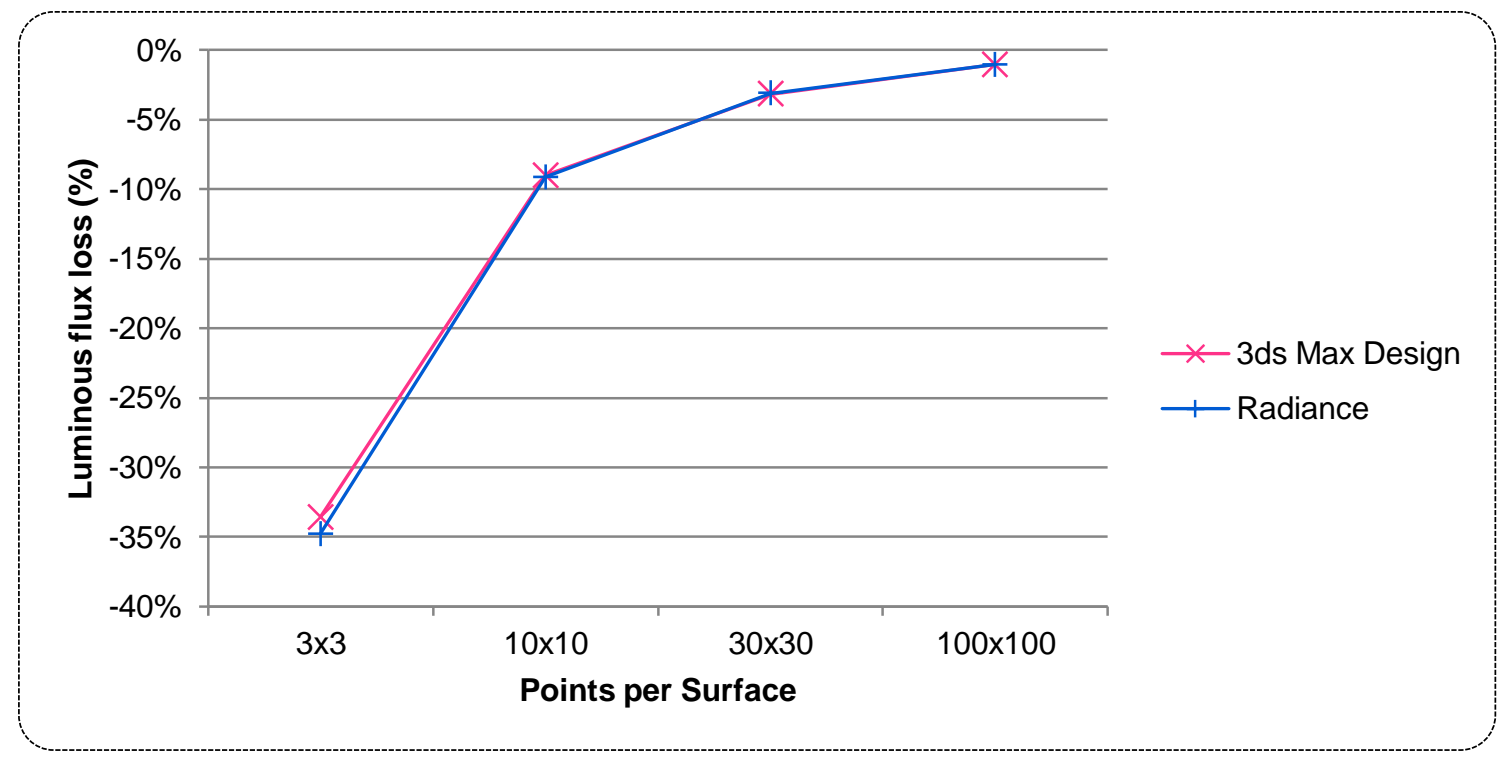


Test case description

Test case 5.5 assesses the ability of a light simulator to consider the directional transmittance of clear glass into consideration; an aspect critical to daylight simulation due to the likelihood of glancing-angle sunlight on windows. The test case consists of a directional parallel light beam aimed at a room opening with a perfectly smooth, $100 \%$ clear glass material covering it. The angle of incidence of the light source is altered in ten degree increments. Measurement points are positioned both in front of and behind the glass material. The analytical values are from an equation for clear glass proposed by Shlick (Shlick, 1993) however it is acknowledged that many other equations for directional transmittance exist.

\section{Modelling issues}

There are two methods to define the surface reflectance of a glass material based on the Arch \& Design material in 3ds Max Design, by Index of Refraction (IOR) and by using a custom curve. Initial testing showed some inaccuracy for the default 'by IOR' method, so the custom curve option was also trialled.

\section{Simulation issues}

Due to the issues in measuring luminous flux documented in the previous test case, instead of measuring the total flux into a room, a single measurement point was used on the opposite side of the glass, which had its normal aligned with the beam of light from the parallel source for each ten degree increment. In this way the directional transmittance can be measured directly rather than via the room's total luminous flux.

\section{Results}

Figure 29 below plots the calculated directional transmittances versus the angle of incidence of the direct light source on the glass plane.

Figure 29: Test case 5.5: Directional transmittance of clear glass ( $T$ ) results

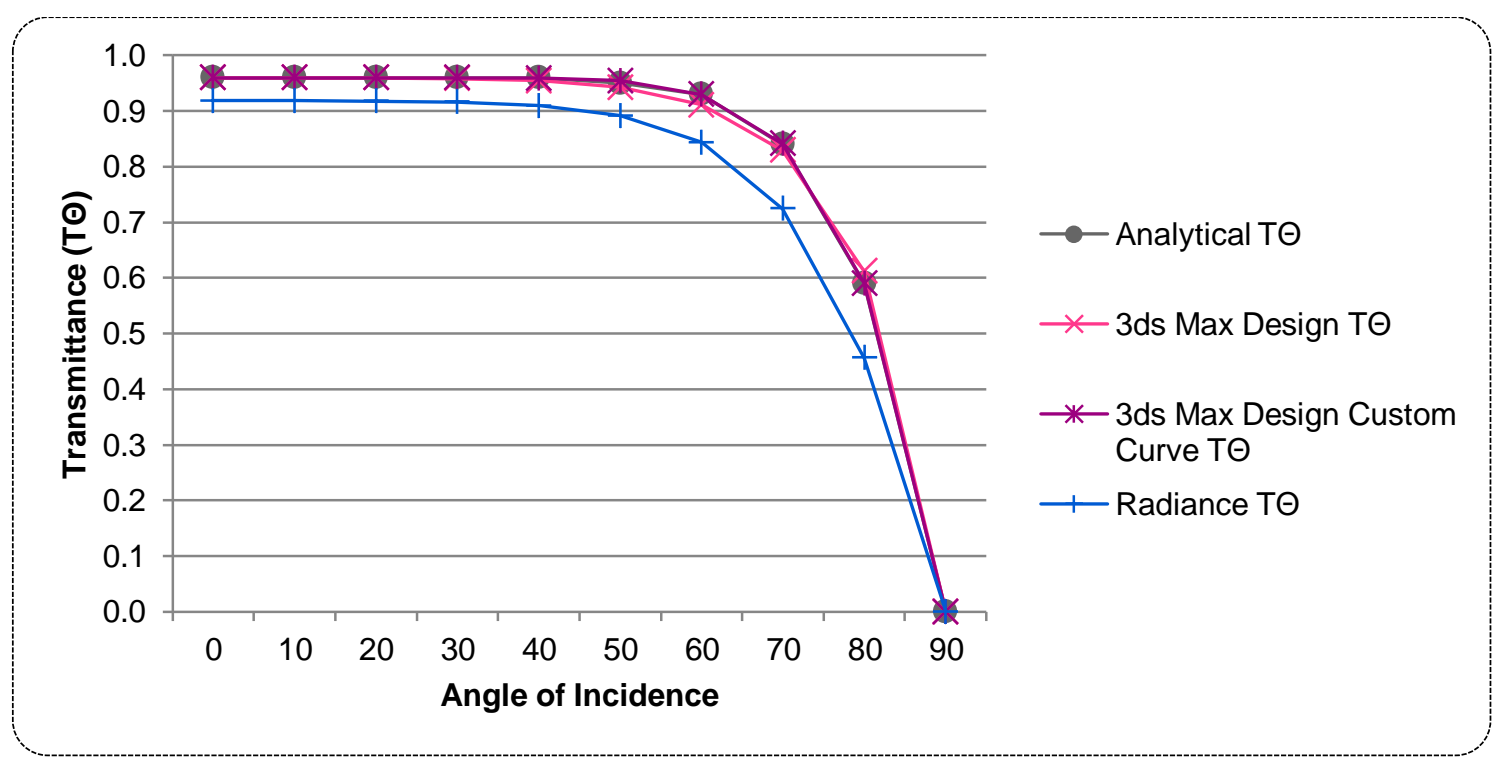

It can be seen that $3 d s$ Max Design produced results near to the analytical reference, however these fell outside of simple rounding error. When using a custom curve to determine the glazing's reflectance however, the match was greatly improved. Radiance uses a more complex equation to determine directional transmittance; an infinite series calculation (Ward, 2004). This equation takes into account the reflection of light from both the inner and outer surfaces of the glass, whereas Shlick's only accounts for the outer surface. This results in Radiance showing twice the reflectance, and therefore double the reduction in transmittance, of 3 ds Max Design at normal incidence. This trend is continued for the remaining angles of incidence. Both simulators are able to produce accurate directional transmittance of glass based on their chosen models, however it is recommended that only the custom curve option be used in 3ds Max Design. 


\subsection{7}

\section{Test case description}

Test case 5.6 consists of three scenarios to test the simulation of light reflection on diffuse surfaces. Each of the scenarios uses a different sized diffuse reflector, illuminated by a parallel light beam from an incident angle of either 35 or 45 degrees. The reflectors are positioned either centred below or next to a set of vertical and horizontal receiving surfaces; labelled $\mathrm{S} 1-\mathrm{V}$ and $\mathrm{S} 1-\mathrm{Hz}$ in figure 30 below. Both receiving surfaces have zero reflectance and are protected from light leaks by an external envelope. The first reflector measures $50 \mathrm{~cm}$ square, centred under the four metre square $\mathrm{S} 1-\mathrm{Hz}$, and is illuminated by a beam at 45 degree and has a reflectance of $80 \%$. The second scenario uses a four metre square, $30 \%$ reflector positioned directly under $\mathrm{S} 1-\mathrm{Hz}$ illuminated from 35 degrees. The third is a 500 metre square reflector of $30 \%$ reflectance next to the two receiving surfaces and illuminated from 45 degrees.

Figure 30: Test case 5.6 geometry \& light source descriptions (CIE TC 3.33, 2005) incident flux $\left(45^{\circ}\right)$ incident flux $\left(35^{\circ}\right)$
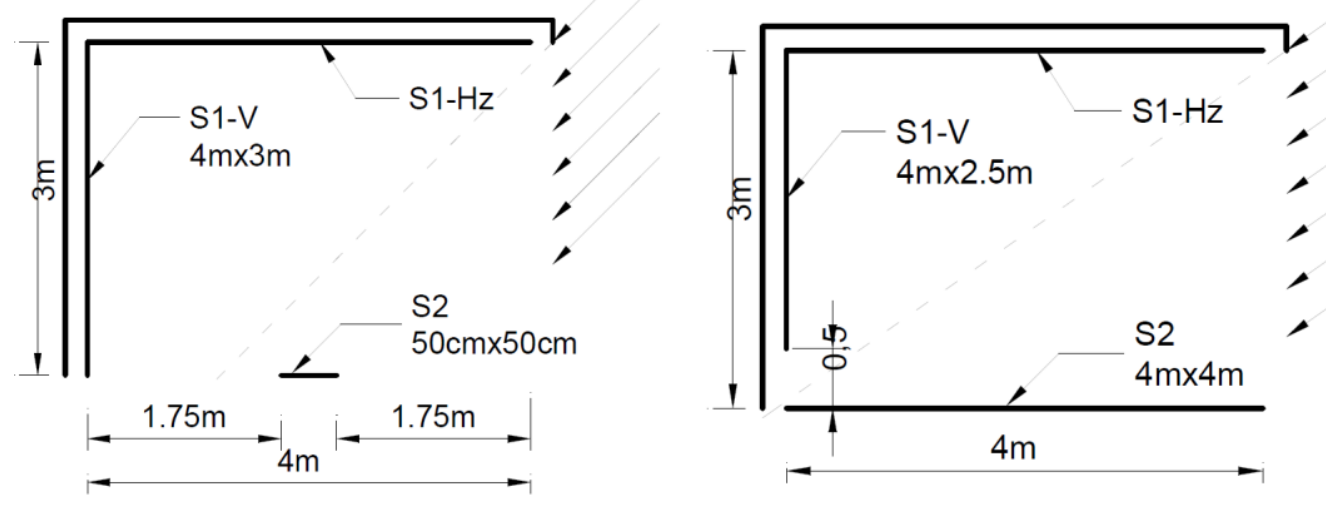

incident flux $\left(45^{\circ}\right)$

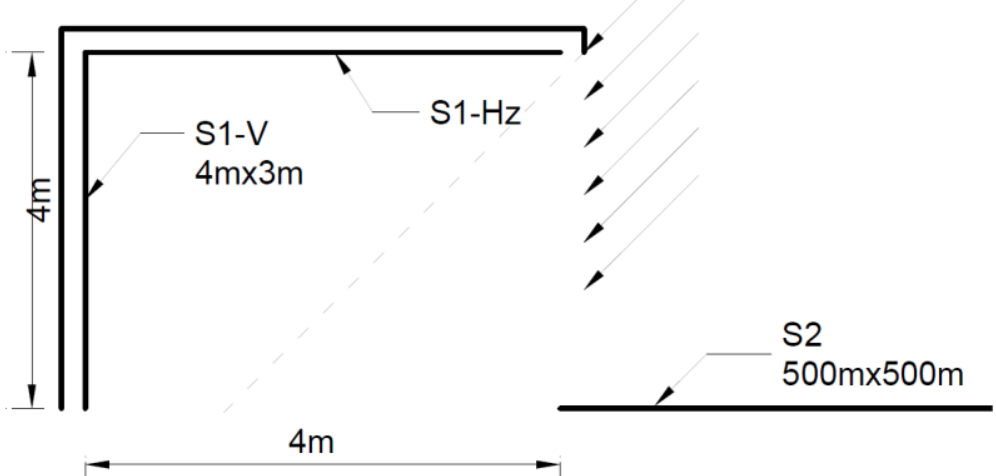

The positions of the measurement points are shown in figure 31 below. Point $A$ is not used in the four by four metre reflector scenario. 


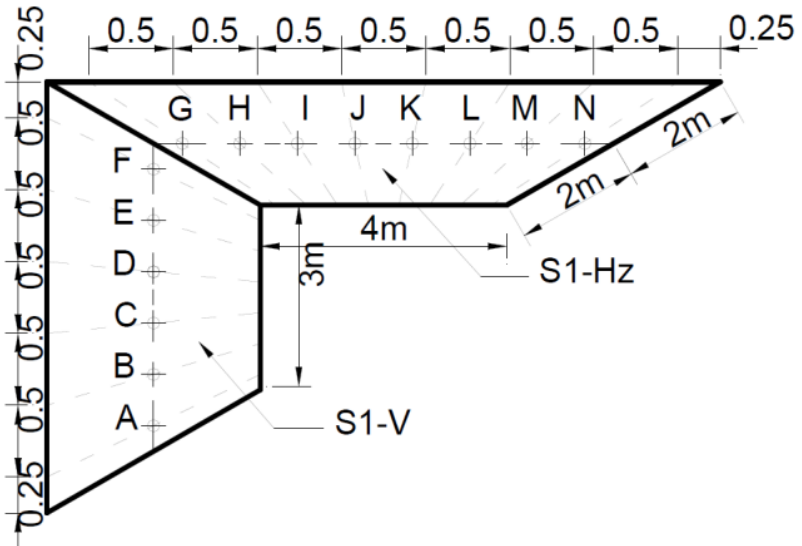

\section{Modelling issues}

There were no issues related to modelling with this test case.

\section{Simulation issues}

There were no simulation parameter issues with this test case.

\section{Results}

Each of the scenarios' results have been presented with the reference points on the horizontal axis and the vertical axis showing the percentage of light reflected from the varying diffuse surfaces. This is calculated by dividing the illuminance at each measurement point by the total horizontal illuminance at the reflector multiplied by its reflectance.

Figure 32: Test case 5.6: Light reflection over diffuse surfaces $(50 \times 50 \mathrm{~cm})$ results

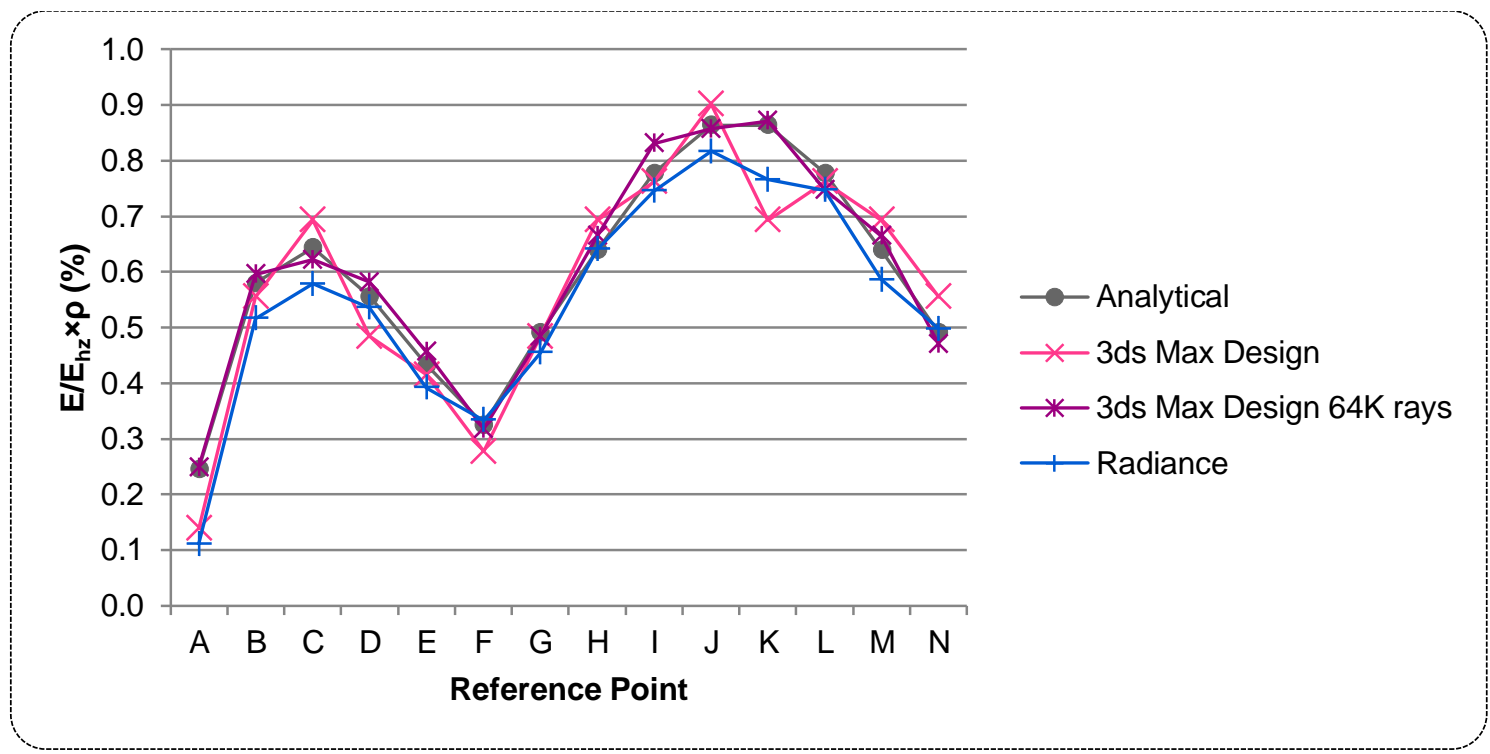

The results in figure 32 above show that even with the relatively high simulation settings used, both simulators struggled to hit the smallest reflecting surface with enough rays to adequately calculate the reflected light. It can be seen that when 3ds Max Design had its Final Gather rays value increased from 2,500 to 64,000 it achieved much better results. However, this came at a significant cost to simulation time. It is common in daylight simulation to replace small, hard to target sources of bounced or refracted light with representative light sources - such as the Daylight Portal objects in 3ds Max Design or a mkillum source in Radiance. This allows the simulator to directly target the light source, instead of treating it like just another piece of geometry. This test case justifies those techniques. 
Figure 33: Test case 5.6: Light reflection over diffuse surfaces $(4 \times 4 \mathrm{~m})$ results

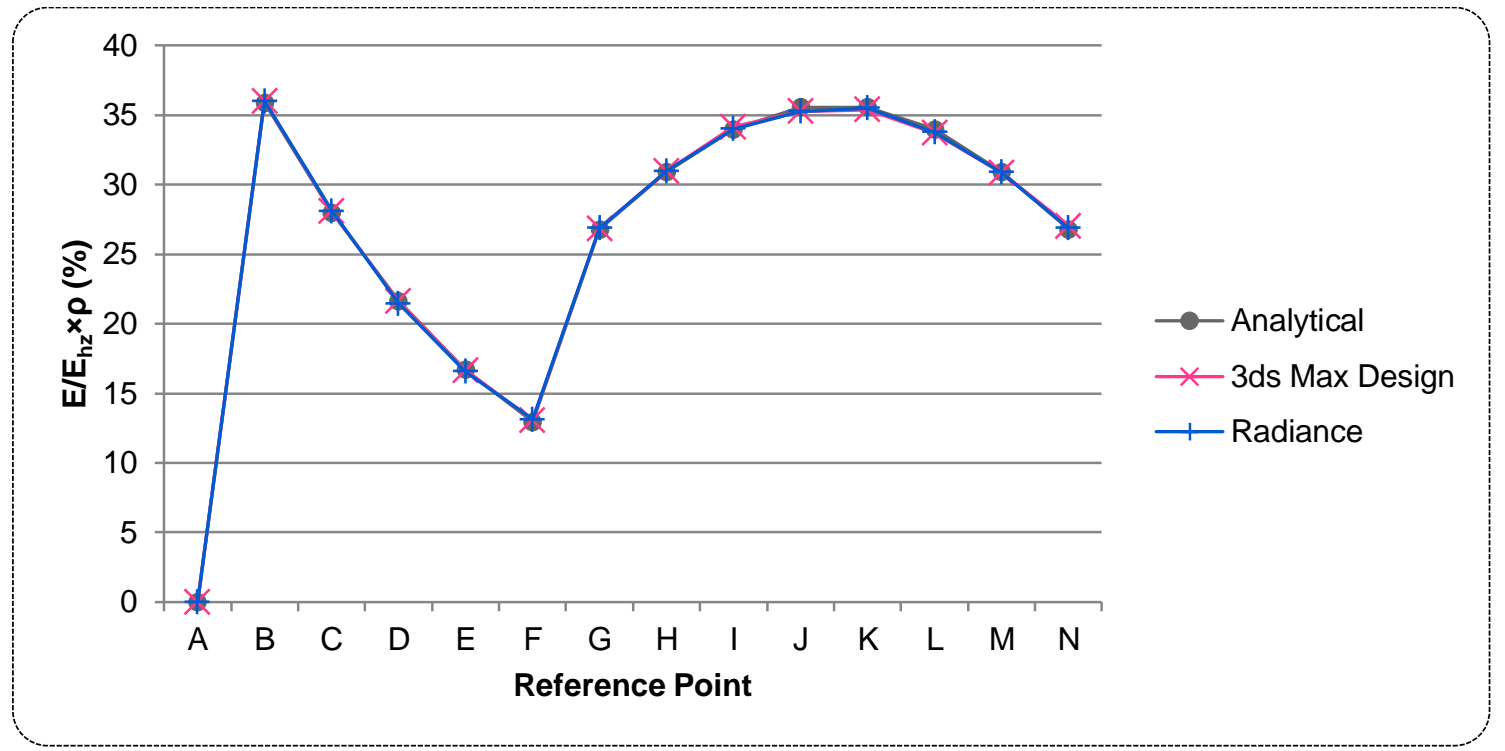

Figure 34: Test case 5.6: Light reflection over diffuse surfaces (50x50m) results

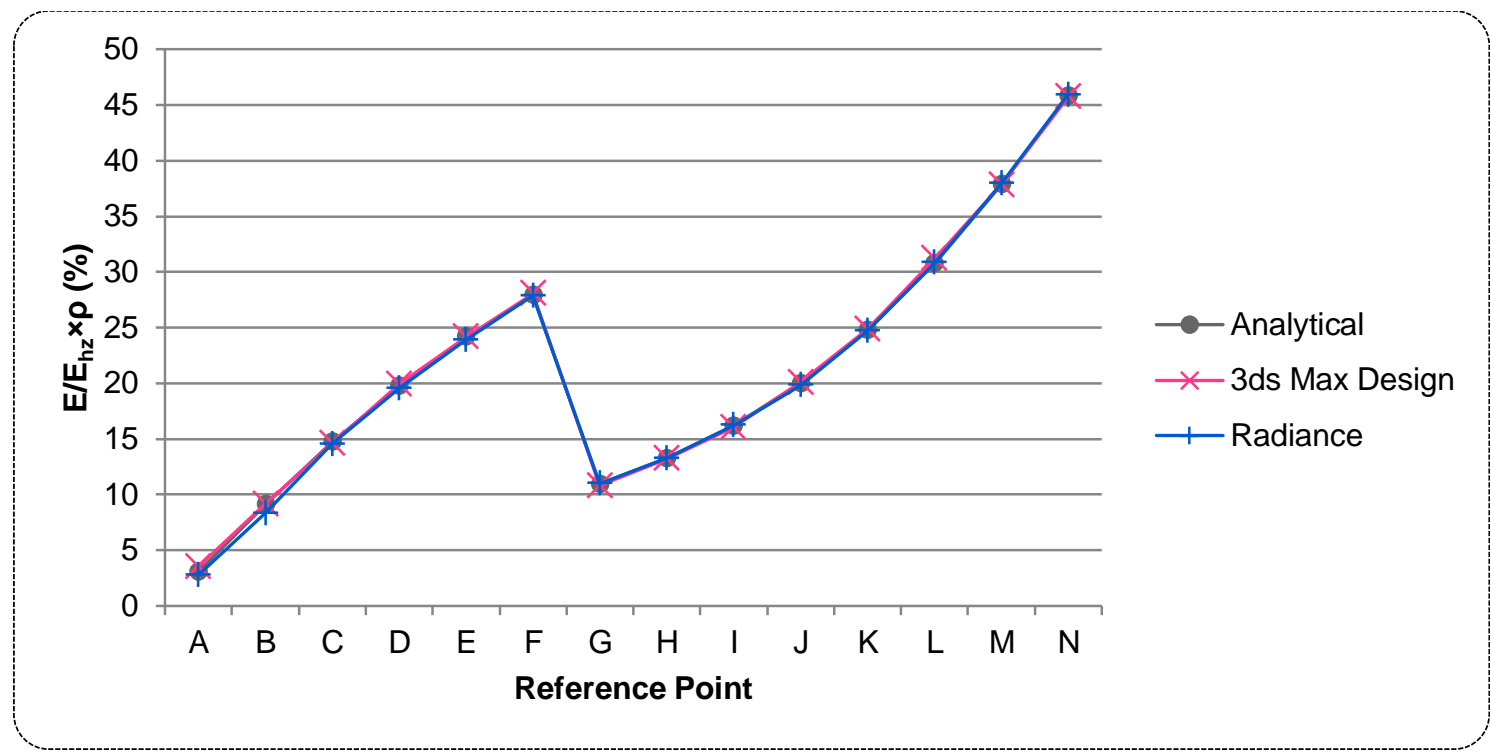

Figure 33 and figure 34 above show the results for the two remaining scenarios of test case 5.6, using the four and 500 metre square reflectors. For both of these scenarios 3ds Max Design and Radiance produced results to within rounding error of the analytical values, suggesting that for medium and large sized diffuse reflecting surfaces, and taking to account the results of the higher simulation settings for the smaller surface, they are correctly simulating diffuse light reflection. This is critical as the vast majority of light interaction in a daylight simulation will be via diffuse reflection. 


\subsection{8}

Test case description

Test case 5.7 is similar to the previous test case involving the diffuse reflection of light, however deals with the influence of an obstruction to the diffuse reflector. Instead of a horizontal reflector this test case uses a vertical surface, S2 in figure 35 below, which bounces light into a room obstructed by a half wall 0.2 metres thick. Each of the measurement points has a different configuration factor relative to the surface, and a different amount of the surface obstructed. In this case, S2 is lit by a direct beam with an incidence angle of sixty degrees.

Figure 35: Test case 5.7 geometry and measurement point description (CIE TC 3.33, 2005)

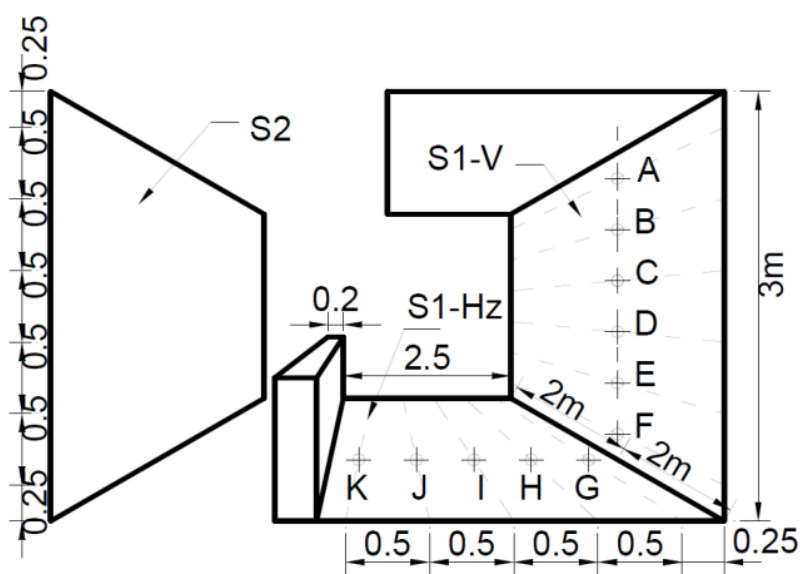

\section{Modelling issues}

There were no issues related to modelling with this test case.

\section{Simulation issues}

There were no simulation parameter issues with this test case.

\section{Results}

The results for this test case are again presented with the reference points on the horizontal axis and the vertical axis showing the percentage of light reflected from the varying diffuse surfaces. This is calculated by dividing the illuminance at each measurement point by the total horizontal illuminance at the reflector multiplied by its reflectance. 


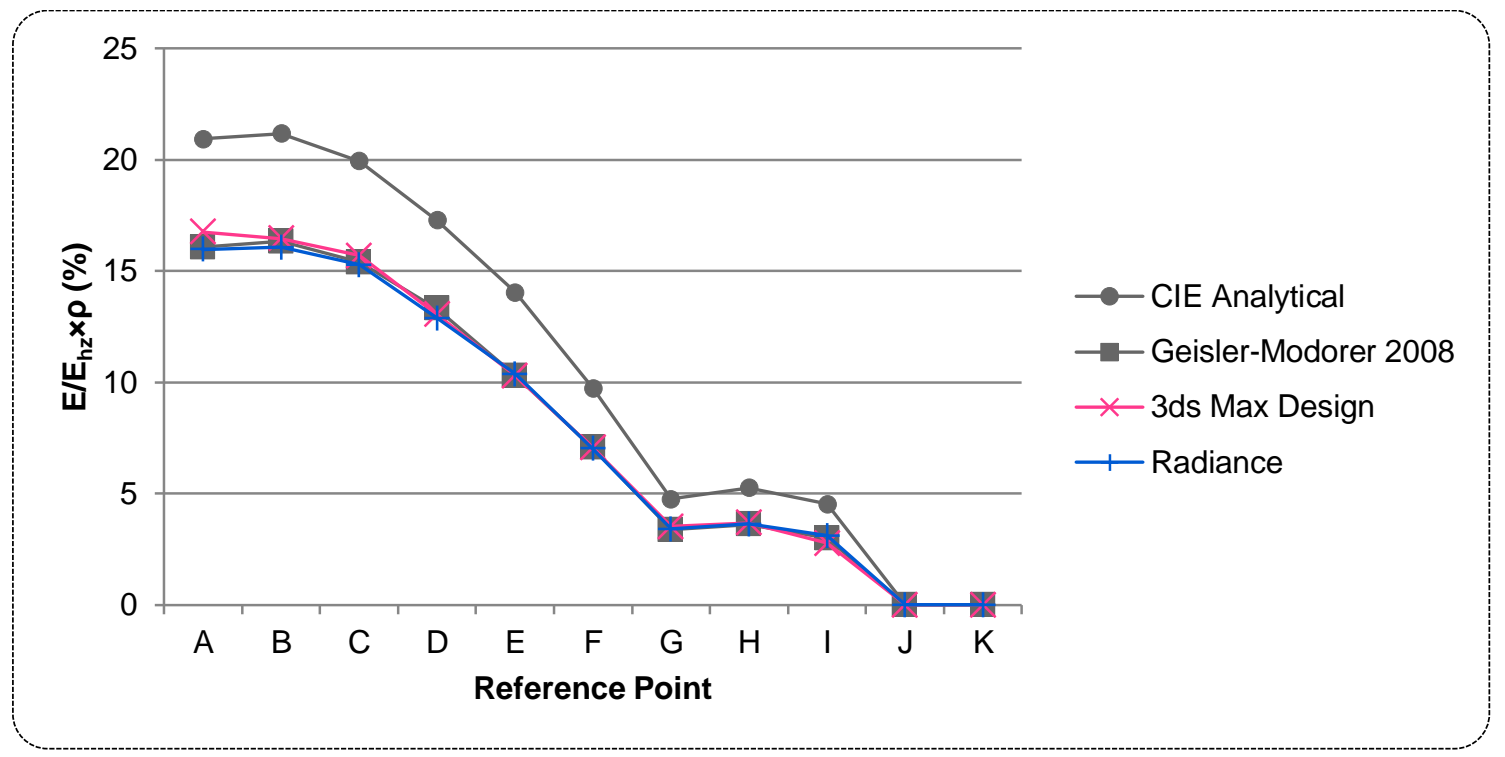

It can be seen from figure 36 above that both 3ds Max Design and Radiance produced results significantly different from the supplied analytical values. However, in a previous, smaller partial validation of Radiance using this test case, these analytical values were shown to be incorrect, and new ones calculated (GeislerMoroder \& Dür, 2008). When compared to Geisler-Moroder's re-calculated analyitcal values, both simulators achieved results to within rounding error. This suggests that both 3ds Max Design and Radiance are correctly dealing with the obstruction to the diffuse source. It has been suggested that an erratum be produced for the CIE 171:2006 document including these new analytcal values for this test case.

\subsubsection{Test case 5.8: Internal reflected component for diffuse surfaces}

\section{Test case description}

This test, based on an integrating sphere, tests the accuracy of diffuse inter-reflections in a room. However, for simplicity in the placement of measurement points, it uses a square room rather than a sphere, which introduces some small error to the analytical values. The room is a cube, measuring four metres on each side and with all surfaces being ideal diffuse reflectors ranging from zero to $95 \%$ reflectivity. At the centre of the room is an isotropic point light source of 10,000 lumens.

\section{Modelling issues}

There were no issues related to modelling with this test case.

\section{Simulation issues}

In testing for this case, it was discovered that for high reflectance values both simulators massively underestimated the room average illuminance. A number of tests were done on the simulation parameters to attempt to improve the results, and the diffuse bounces parameter was determined to be the most critical. The results below show a number of different values for the diffuse or ambient bounce parameter.

\section{Results}

The results for this test case are plotted in figure 37 below with the variations of room reflectance on the horizontal axis and the room average illuminance on the vertical axis. The 3ds Max Design and Radiance $6 \mathrm{~b}$ lines are for the standard set of simulation parameters described earlier in this report, and available in full in appendix 1 . The remaining lines are for identical simulation parameters excepting the diffuse or ambient bounce value, which has been tested at 8, 16, 32, 64 and 128 bounces for each simulator. 
Figure 37: Test case 5.8: Internal reflected component for diffuse surfaces results

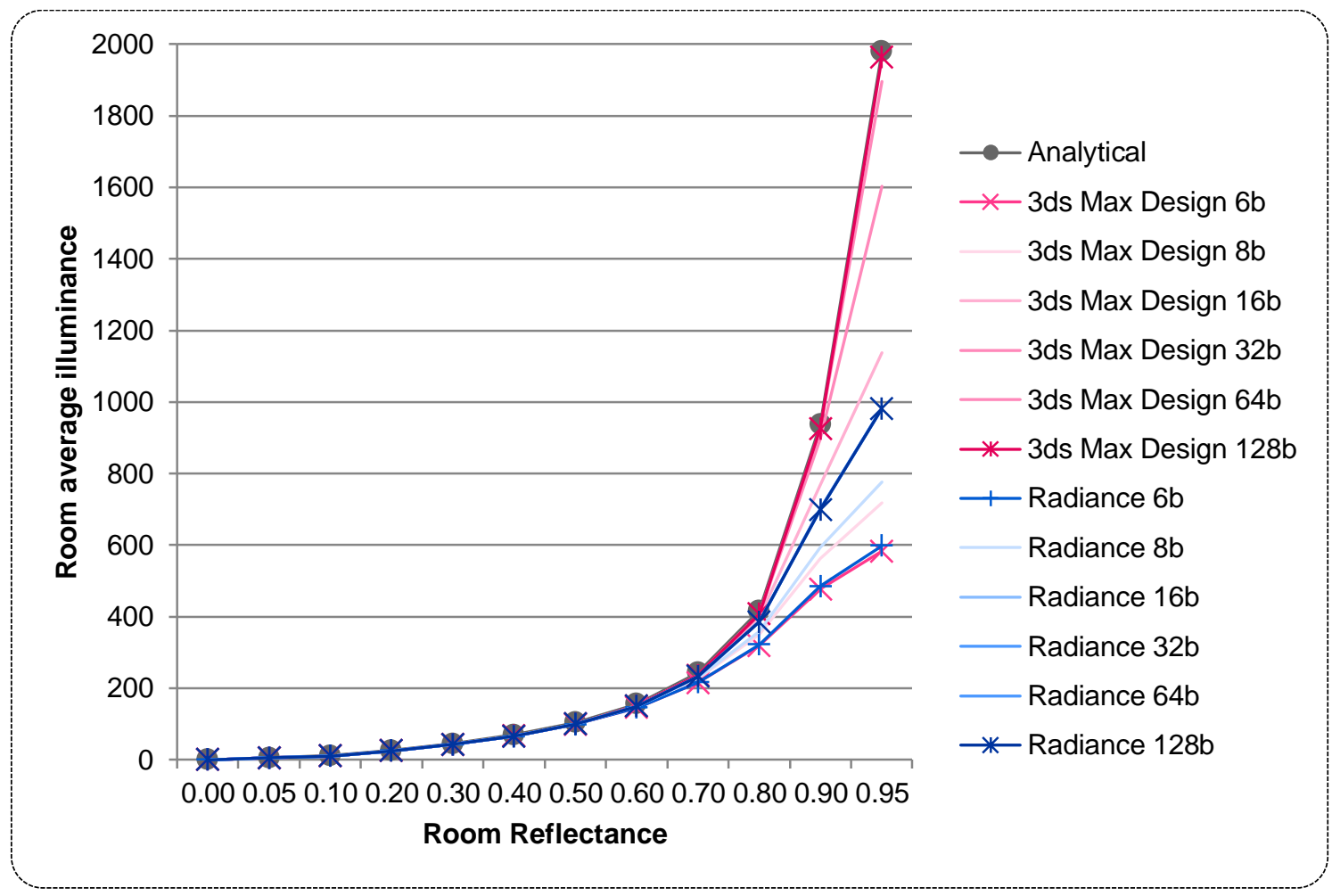

The results show that for both simulators, diffuse inter-reflections are simulated accurately using the base settings until the room reaches a reflectance of 0.7 . From this point on, the traces begin to diverge. It required 128 diffuse bounces for 3ds Max Design to reach a number close to the analytical value; but never came within rounding error. No further gains were found for 256 bounces, suggesting either $3 d s$ Max Design silently limits bounces to 128 , or the analytical values are slightly too high stemming from their origins in an integrating sphere rather than a cube. It is suspected the latter, as similar findings were found in a recent validation of the AGi32 software (Dau Design and Consulting Inc., 2007). Radiance shows no increases in accuracy beyond 16 bounces, as the 16, 32, 64 and 128 bounce traces all overlap. It is known that Radiance begins to reduce the rays cast as the ambient bounces value increases past 8 to save simulation time, hence the limit reached at or before 16 bounces.

Critically these results suggest that if a designer is simulating a space where the average room reflectance is above 0.7 , they should look to increase their ambient bounce limit to something past what is normally considered. This lends credence to the suggestion that all designers using simulation software should conduct a sensitivity analysis for each project they work on - perhaps not only for their model inputs but simulation parameters as well. 


\section{Test case description}

These two test cases are aimed at testing the capability of a lighting simulator to calculate the sky component under different sky conditions. As 3ds Max Design only has the ability to model the CIE Overcast and Clear skies, testing has been limited to just the CIE Overcast sky from the different analytical values available. These tests are important as the Daylight Factor is a commonly used parameter for determining daylight availability inside a building (CIE TC 3.33, 2005), and is usually calculated using the CIE Overcast sky. Both test cases use the same four metre square room and measurement points, pictured below in figure 38. All internal surfaces have zero reflectance. Test case 5.9 uses a one metre square opening centred in the ceiling, which has zero thickness. Test case 5.10 adds a clear glass pane to the opening.

Figure 38: Test case 5.9 \& 5.10 geometry \& measurement point description (CIE TC 3.33, 2005)

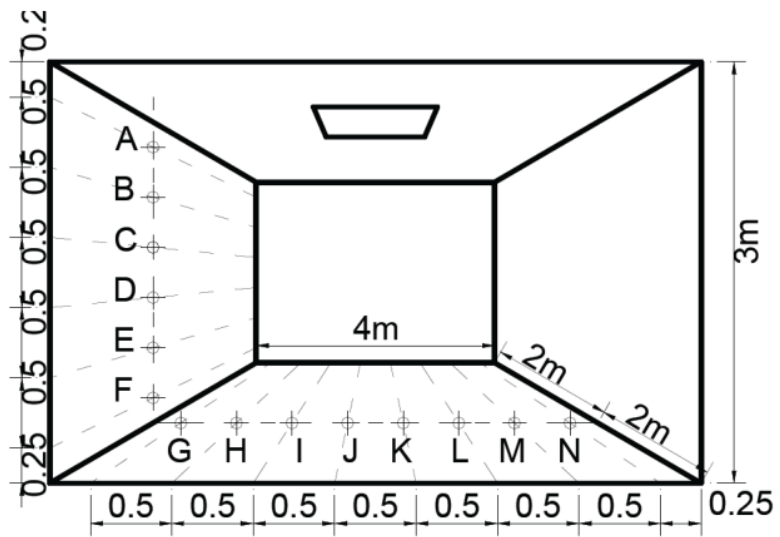

\section{Modelling issues}

There were no issues related to modelling with this test case.

\section{Simulation issues}

There were no simulation parameter issues with this test case.

\section{Results}

The results for test case 5.9 and 5.10 follow the same format, with the sky component in percentage (equal to the Daylight Factor in these cases) on the vertical axis plotted for each reference point on the horizontal axis. 
Figure 39: Test case 5.9: Sky component for a roof unglazed opening results

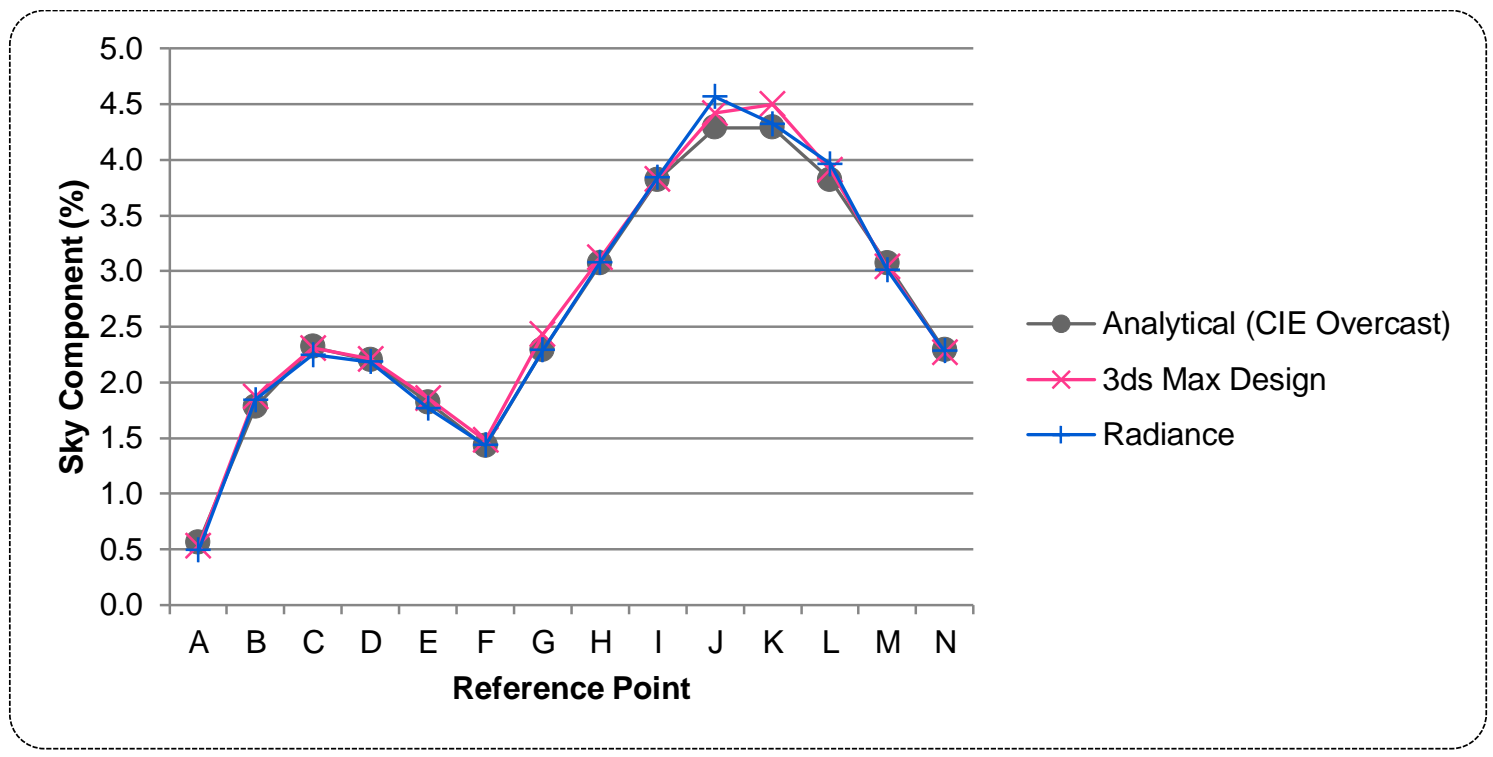

Figure 40: Test case 5.10: Sky component for a roof glazed opening results

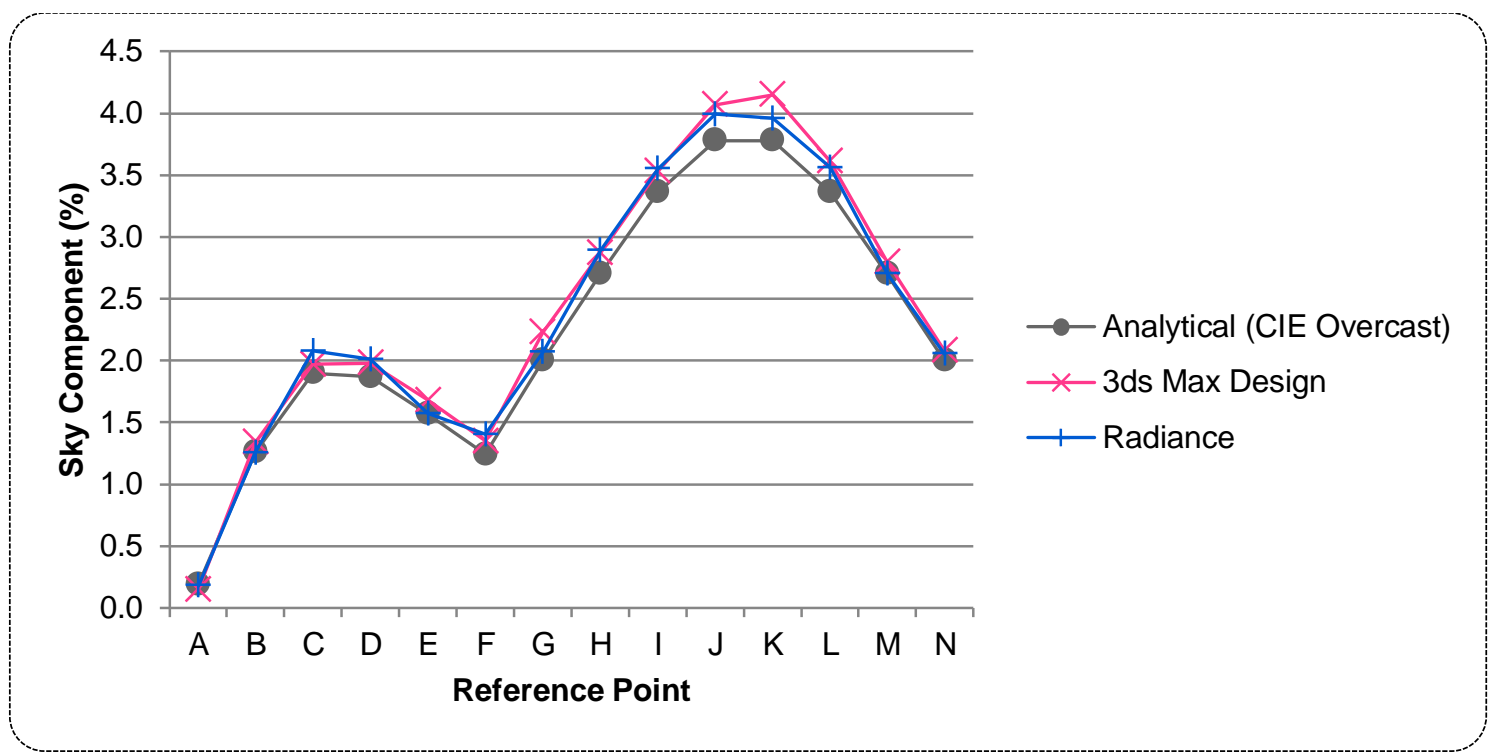

The results in the figures above show what at first looks like a poor match for both simulators in both cases. However, the largest differences between analytical value and simulated result equate to less than $0.25 \%$ in Daylight Factor for test case 5.9 and less than $0.5 \%$ for test case 5.10 with the addition of glazing - differences that are unlikely to have a significant impact on design. Differences are for the most part within $0.1 \%$, which is within rounding error. It is therefore considered that both simulators have achieved sufficient accuracy for these test cases. 


\section{Test case description}

These two test cases are intended to test not only the sky component as the previous two test cases, but also include the external reflected component from a ground plane. The cases both use similar geometry; a four metre square room with a three metre high ceiling. There is a two metre wide by one metre high opening in one wall which has zero thickness. For test case 5.12 this opening is covered by a clear glass pane $6 \mathrm{~mm}$ thick. All room surfaces have zero reflectances, and the ground plane is $30 \%$ reflective. The cases are illuminated by the CIE Overcast general sky. The measurement points are shown in figure 41 below.

Figure 41: Test case 5.11 \& 5.12 geometry \& measurement point description (CIE TC 3.33, 2005)

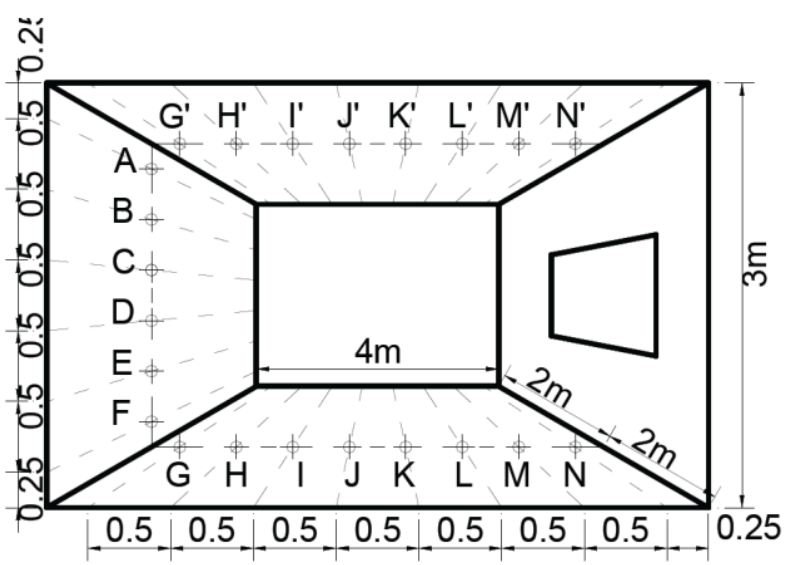

\section{Modelling issues}

There were no issues related to modelling with this test case.

\section{Simulation issues}

There were no simulation parameter issues with this test case.

\section{Results}

The results for these two test cases in figure 42 and figure 43 below show, sky component and external reflected component (calculated as Daylight Factor) plotted for each reference point. Points $\mathrm{E}$ through $\mathrm{N}$ contain only sky component, $\mathrm{C}$ and $\mathrm{D}$ a mixture of both, and $\mathrm{A}, \mathrm{B}$ and $\mathrm{G}$ ' through $\mathrm{N}$ ' having only external reflected components. 
Figure 42: Test case 5.11: Sky component and external reflected component for a façade unglazed opening results

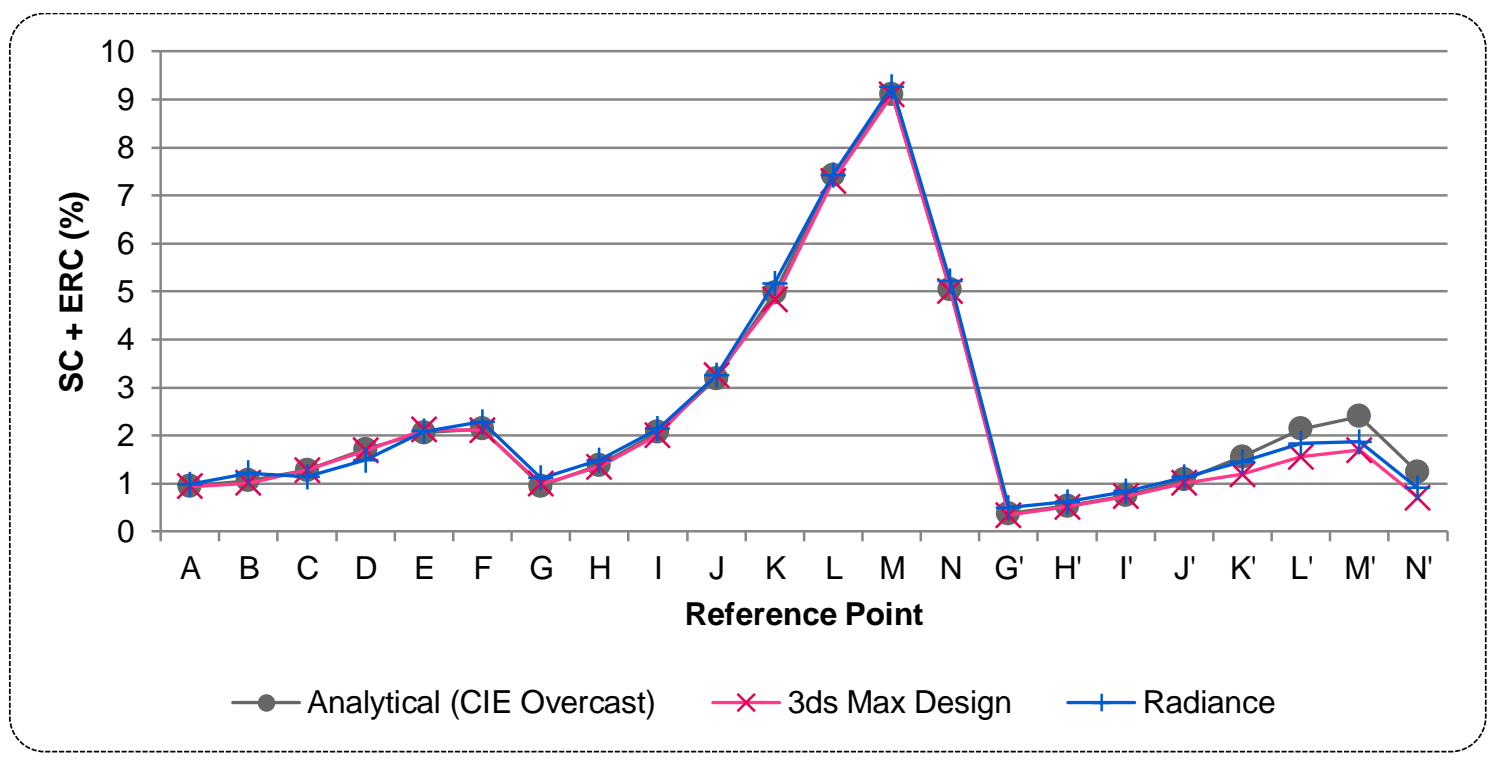

Figure 43: Test case 5.12: Sky component and external reflected component for a glazed façade opening results

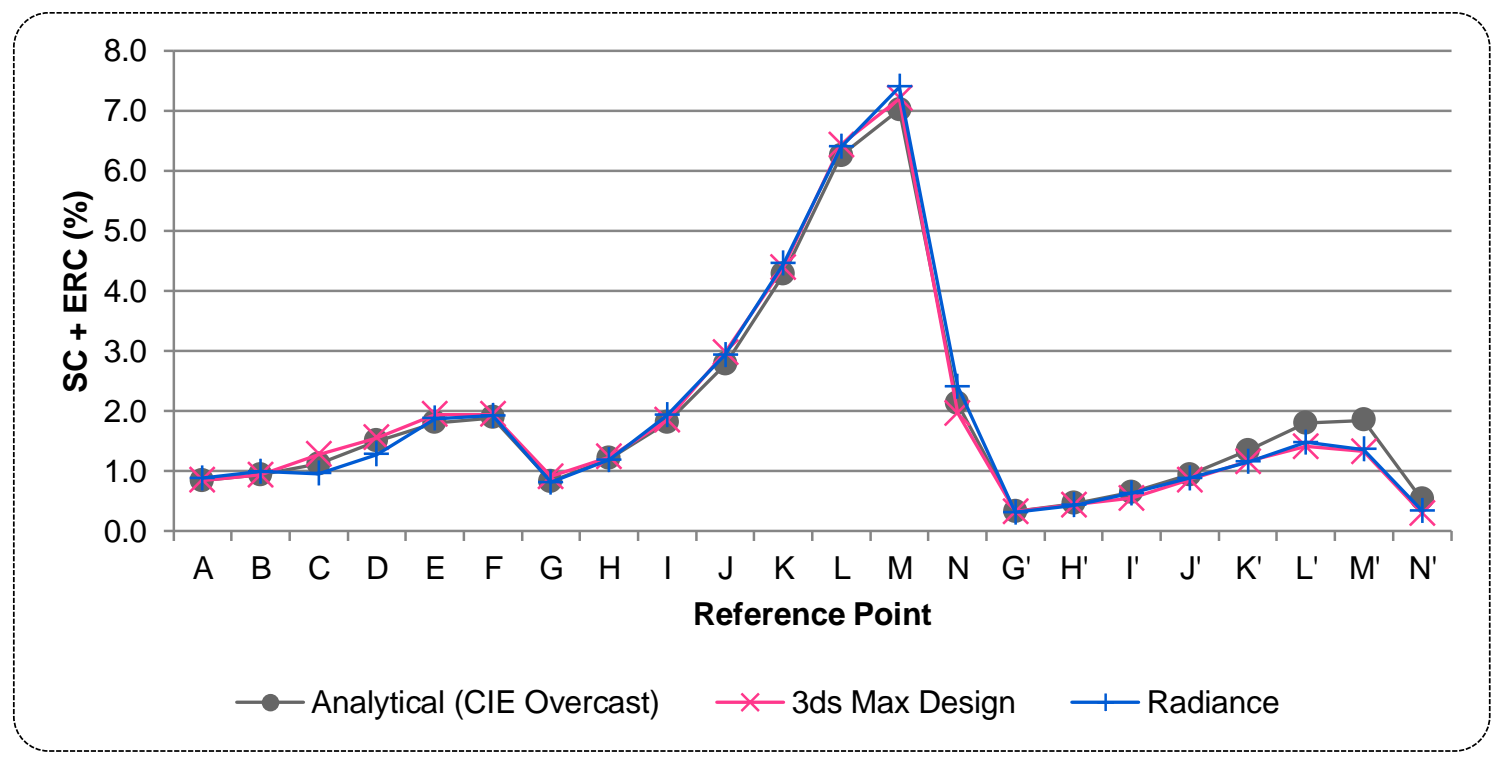

The results for these two cases show that for the majority of measurement points both simulators produced predictions that were within rounding error. However, there is a trend for the points dealing with the external reflected component to be lower than the analytical values; particularly for those exposed to the ground plane nearest to the test room. It was determined that this was due to the analytical values not taking into account the shading effect of the room on the ground plane. Without this being accounted for, this test case can only be used for the points exposed to the sky. It can however be determined that both simulators are correctly simulating sky components through glazed and unglazed façade openings. 


\section{Test case description}

Test cases 5.13 and 5.14 again use a four metre square, three metre high room, with a ground plane, with the addition of various sized horizontal and vertical obstructions respectively, as shown in figure 44 and figure 45 below. They are designed to test the ability of a lighting program to simulate the influence of external masks to internal illuminance. Both cases assume that the masks have a uniform luminance - in affect light sources themselves. The cases could then be considered a combination of the previous test case (5.11) and test case 5.3 - area lights, rather than a unique scenario.

Figure 44: Test case 5.13 geometry description (CIE TC 3.33, 2005)

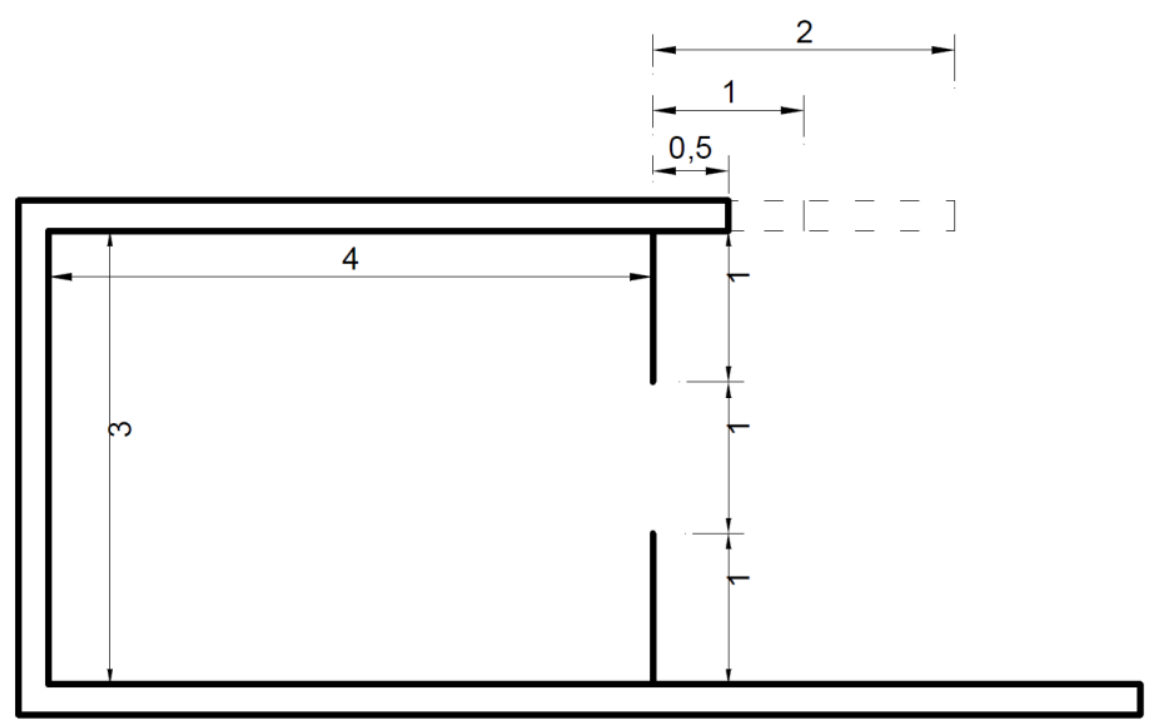

Figure 45: Test case 5.14 geometry description (CIE TC 3.33, 2005)

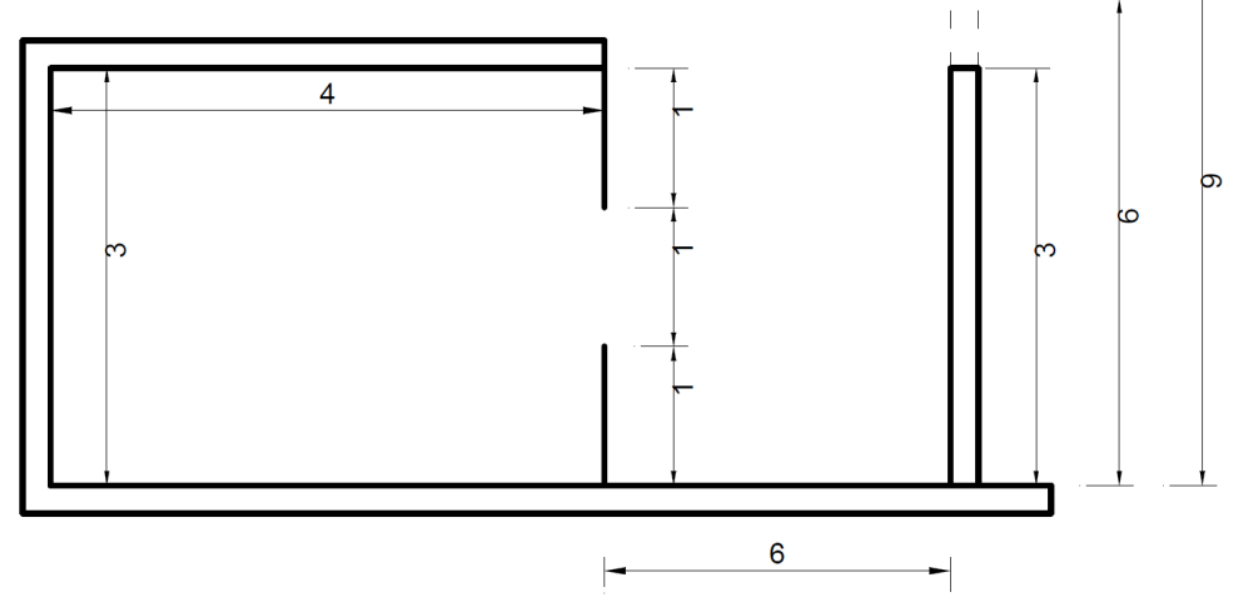

\section{Modelling issues}

There were no issues related to modelling with this test case.

Simulation issues

There were no simulation parameter issues with this test case. 
Results

No results have been given for these two test cases, as the provided analytical values do not take into account many factors:

- The inter-reflection between the masks and ground plane;

- The shading effects of the masks on the ground plane;

- The shading effect of the room on the ground plane;

- That in order for the masks to be of uniform luminance they must ignore contributions from the ground plane, and be made light sources themselves.

These factors greatly affect the measured Sky and External Reflected Components, such that they cannot be reasonably compared to the reference data. It can therefore be stated that test cases 5.13 and 5.14 are invalid in their current form, and should be reconsidered.

\subsubsection{Test case 6.1: Sun patches}

\section{Test case description}

This test case is intended to verify the capability of a program to produce accurate sun patches. The test case is proposed as a square room receiving sunlight through a rectangular façade opening with a given incidence angle. For this case, a four metre square room has been used, with a one metre square opening in the centre of one wall of zero thickness, as pictured in figure 46 below. All surfaces have zero reflectance. A parallel (sun) light beam of 10,000 lux is incident at sixty degrees, creating a sun patch on the room's floor. The sun patch can be analytically calculated to be a rectangle measuring one metre by 0.577 metres, and have an average illuminance of $10,000 \cos (60) ; 8660$ lux.

Figure 46: Test case 6.1 description (author's graphic)

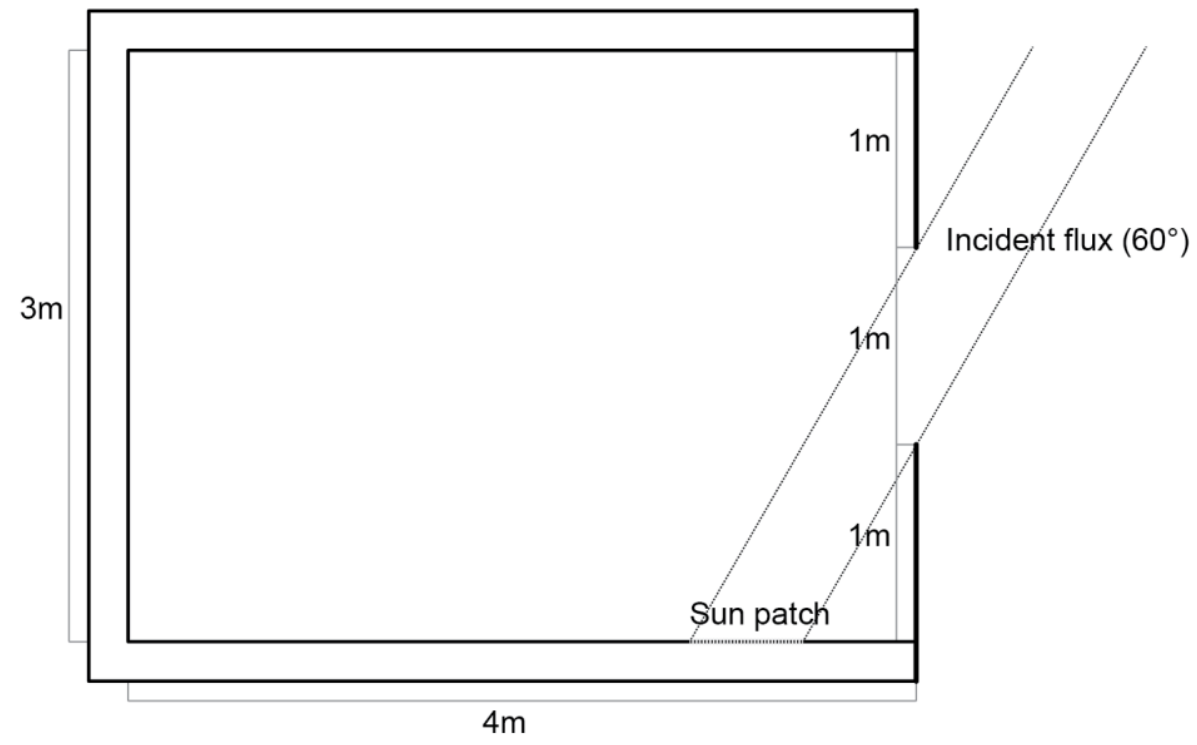

\section{Modelling issues}

There were no issues related to modelling with this test case.

\section{Simulation issues}

There were no simulation parameter issues with this test case.

\section{Results}

Both 3ds Max Design and Radiance correctly produced sun patches of the correct size, to within $0.25 \mathrm{~mm}$. This was tested through a series of measurement points at $0.25 \mathrm{~mm}$ intervals over the boundary of the sun patch on each side. Both simulators also achieved average illuminance values of 8660 lux, to within rounding error. 


\section{Test case description}

Test case 6.2 aims to test the capability of a program to simulate specular reflections. The geometry and lighting conditions for this test case are identical to that of test case 6.1, except the floor of the room has been replaced with a theoretical perfect specular reflector or mirror. This causes the sun patch to be directly translated on to the ceiling of the room as shown in figure 47 below, and allows it to be measured in the same fashion as the original sun patch in test case 6.1.

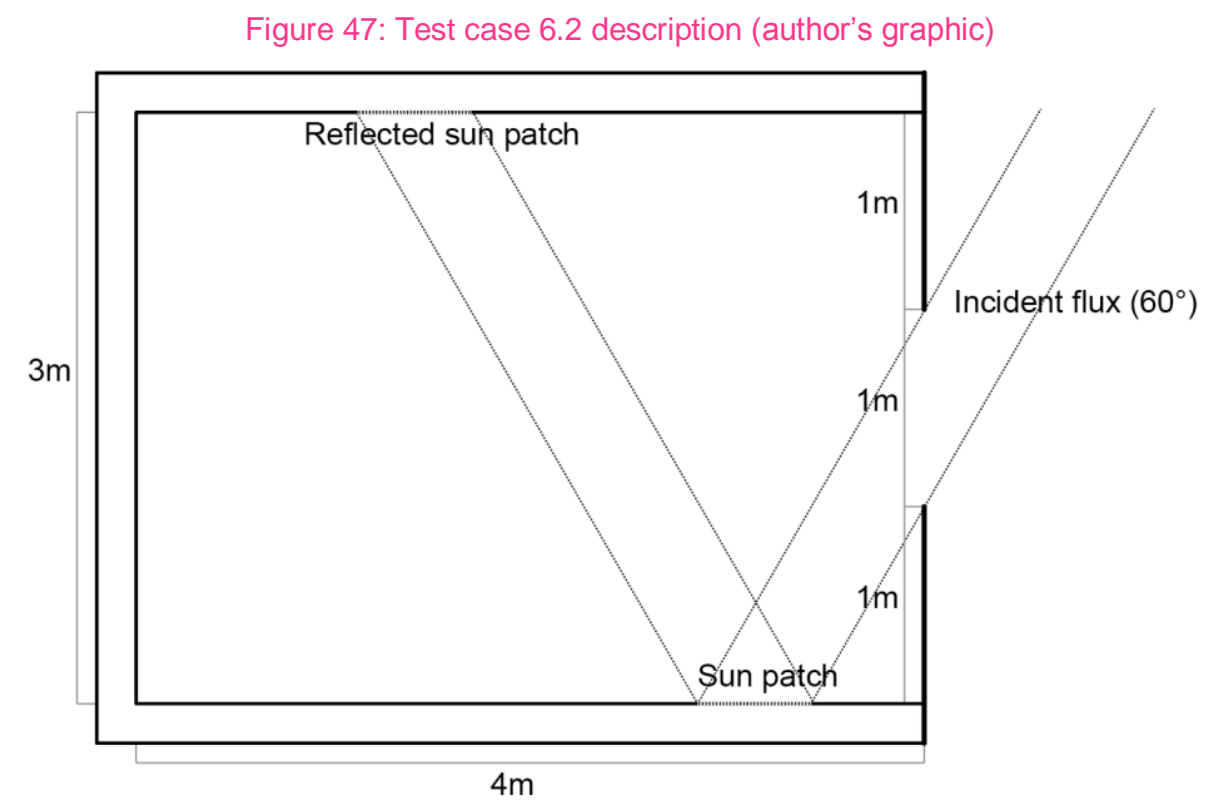

\section{Modelling issues}

There were no issues related to modelling with this test case.

\section{Simulation issues}

In order for 3ds Max Design to properly simulate specular reflections, it requires forward raytracing (GI) and caustics to be switched on. These settings however are not considered valid for the lighting analysis functions of the software. This means that while it may be able to produce accurate-looking images of specular reflections, it is not able to accurately account for them in lighting simulations.

\section{Results}

For test case 6.2 3ds Max Design was unable to correctly simulate the specular reflection of a sun patch onto a ceiling using its lighting analysis tools. Instead, it returned values equal to the configuration factor of the sun patch as if it were a uniform diffuse reflector, as described for test case 5.6 in section 4.1.7. However, using its forward raytracing (Gl) and Caustics engines, it was possible to create images of the specular-reflected sun patch. However, this method has been shown to be unable to correctly simulate daylight due to it not considering multiple bounces of the diffuse portion of the sky.

Radiance however was able to correctly simulate the reflected sun patch in size and illuminance, however not consistently. Occasionally the program would "miss" the sun when casting rays, and return a value of zero. The incidence of this can be somewhat reduced by increasing the simulation parameters; however this will never fully remedy the issue.

This test case shows that for lighting scenarios involving specular reflections 3ds Max Design must not be used, and Radiance should be used with care. 


\subsubsection{Test case 6.3: Light transmission through diffuse glazing}

Test case description

This test is intended to assess a program's ability to simulate an ideal diffuse glass material. There is no complete test case description for this test case; only a proposal that one be developed for inclusion within the test cases using configuration factor equations.

Modelling issues

There were no issues related to modelling with this test case.

\section{Simulation issues}

There were no simulation parameter issues with this test case.

\section{Results}

This test case was not undertaken, as without a standard test procedure the goal of the test cases of enabling the comparison of simulators cannot be met. Additionally, a test case involving a partially diffuse glass material, more likely to be found in practice, could also be recommended. The development of such test cases is considered future work.

\subsubsection{Test case 6.4: Light transmission through bi-directional glazing}

\section{Test case description}

This test is intended to assess a program's ability to simulate the bi-directional reflection effect of glazing materials. There is no complete test case description for this test case; only a proposal that one be developed for inclusion within the test cases using a standardised Bi-directional Transmittance distribution Function (BTDF). Without this function, it is not possible to develop analytical values for comparison.

\section{Modelling issues}

There were no issues related to modelling with this test case.

\section{Simulation issues}

There were no simulation parameter issues with this test case.

\section{Results}

This test case was not undertaken, as without a standard test procedure the goal of the test cases of enabling the comparison of simulators cannot be met. The development of such a procedure is considered future work. 


\section{Test case description}

This test is intended to assess a program's ability to simulate the bi-directional reflection effect of a surface. There is no complete test case description for this test case; only a proposal that one be developed for inclusion within the test cases.

\section{Modelling issues}

There were no issues related to modelling with this test case.

\section{Simulation issues}

There were no simulation parameter issues with this test case.

\section{Results}

This test case was not undertaken, as without a standard test procedure the goal of the test cases of enabling the comparison of simulators cannot be met. The development of such a procedure is considered future work.

\subsubsection{Test case 6.6: Spectral calculation}

\section{Test case description}

Test case 6.6 is designed to test the spectral absorption of a surface. The case consists of a light source of a discontinuous spectrum illuminating an ideal diffuse surface of the opposite selective absorption. A measurement point directly facing the receiving surface detects whether any light is reflected.

\section{Modelling issues}

There were no issues related to modelling with this test case.

\section{Simulation issues}

There were no simulation parameter issues with this test case.

\section{Results}

The results for case 6.6 are presented in table 4 below. They show that for all spectral combinations both simulators are respecting the spectral absorption properties of the receiving surface.

Table 4: Test case 6.6: Spectral calculation results

\begin{tabular}{|l|l|l|l|l|l|l|l|}
\hline \multicolumn{4}{|c}{ Light Source Colour } & \multicolumn{3}{c|}{ Reflector Colour } & \multicolumn{2}{c|}{ Reflected Illuminance Received } \\
\hline R & G & B & R & G & B & 3ds Max Design & Radiance \\
\hline 1.0 & 0.0 & 0.0 & 0.0 & 1.0 & 1.0 & 0.0 & 0.0 \\
\hline 0.0 & 1.0 & 0.0 & 1.0 & 0.0 & 1.0 & 0.0 & 0.0 \\
\hline 0.0 & 0.0 & 1.0 & 1.0 & 1.0 & 0.0 & 0.0 & 0.0 \\
\hline 1.0 & 1.0 & 0.0 & 0.0 & 0.0 & 1.0 & 0.0 & 0.0 \\
\hline 1.0 & 0.0 & 1.0 & 0.0 & 1.0 & 0.0 & 0.0 & 0.0 \\
\hline 0.0 & 1.0 & 1.0 & 1.0 & 0.0 & 0.0 & 0.0 & 0.0 \\
\hline
\end{tabular}


Test case description

This test case is intended to examine the ability of a simulator to produce valid illuminances from the CIE general sky types. It proposes that the zenith luminance, direct normal illuminance, and global horizontal and vertical illuminances be used as reference values. The same test could be proposed for the Perez sky model.

Modelling issues

There were no issues related to modelling with this test case.

\section{Simulation issues}

There were no simulation parameter issues with this test case.

Results

Both 3ds Max Design and Radiance have been shown to correctly reproduce the CIE general skies (just the Clear and Overcast for 3ds Max Design) and Perez all-weather model. This is unsurprising considering the extensive validation of Radiance for both sky types, and 3ds Max Design's certification for use in calculating the LEED EQ 8.1 daylighting credit (using the CIE models) and Reinhart's 2009 validation under the Perez model.

\subsubsection{Test case 6.8: Daily and monthly variation of external illuminance}

\section{Test case description}

This test case is undefined by the CIE 171:2006 document; however it is intended to assess the capability of a program to correctly alter the sun trajectory for a given sky model through date, time and location inputs.

\section{Modelling issues}

There were no issues related to modelling with this test case.

\section{Simulation issues}

There were no simulation parameter issues with this test case.

\section{Results}

Both 3ds Max Design and Radiance have the capability to input either solar altitude and azimuth or date, time and location into the CIE general sky types and the Perez -all weather sky model. 3ds Max Design also has the ability to input an Energy Plus Weather (EPW) Typical Meteorological Year (TMY) format weather file. This makes them ideally suited for daylight simulations using real data. 


\section{Test case description}

This test case is intended at identifying the potential for light leaks through or along geometry via interpolation that occurs in both ray tracing (as in 3ds Max Design and Radiance) and radiosity light simulation techniques. The test case consists of a sealed box illuminated by a bright sun and sky light. For this test case the room modelling technique described in previously in section 2.4 was used to create the box, along with the entire box as one object, and with six separate $100 \mathrm{~mm}$ thick boxes. A 100,000 lux light source was used.

\section{Modelling issues}

There were no issues related to modelling with this test case.

\section{Simulation issues}

There were no simulation parameter issues with this test case.

\section{Results}

In all modelling cases both 3ds Max Design and Radiance produced average internal illuminances below 0.1 lux, considered to be an acceptable result. No false shadows were observed. Figure 48 below shows two false-colour illuminance renders from 3ds Max Design. Blue is zero lux, and red corresponds to one lux, an extremely tight scale. On the left is the typical result seen for all cases when no light meter object was present. The image on the right shows an anomaly where adding a light meter object placed one millimetre above the floor caused a small light leak, "sucking" some illuminance through the floor in an unpredictable pattern, likely due to an interpolation error. However, this only amounted to one lux, not a significant amount given the opposite side of the surface was illuminated to 20,000 lux.

Figure 48: Test case 6.9 results; 3ds Max Design false-colour illuminances
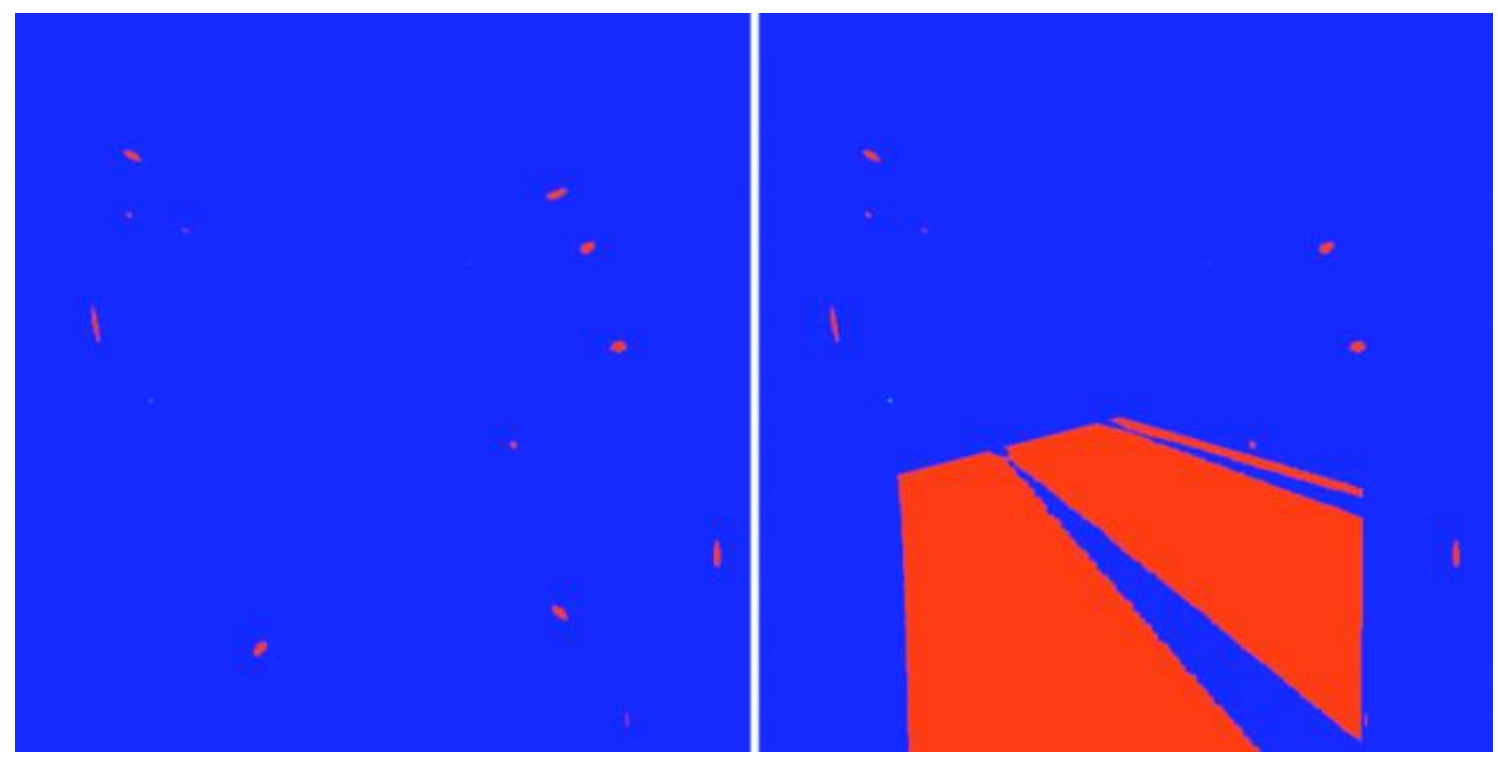

Despite this error, both 3ds Max Design and Radiance have demonstrated they are capable of simulating without light leaks provided a proper modelling procedure is followed, as a one lux error is unlikely to ever be significant in a daylight simulation. 


\section{Test case description}

This test case consists of a cubic room of four metre sides, with an isoradiant point source placed at the centre. Ten by ten grids of measurement points are placed on each of the six surfaces. The test case is run twice; with the room reflectance first at $0 \%$ and then at $50 \%$. In order to determine if all surfaces are symmetrical, the corresponding points for each grid should match exactly.

\section{Modelling issues}

There were no issues related to modelling with this test case.

\section{Simulation issues}

There were no simulation parameter issues with this test case.

\section{Results}

For the zero reflectance scenario, both 3ds Max Design and Radiance produced precisely symmetrical results to within rounding error. For the $50 \%$ reflectance scenario, some points drifted by a maximum of one lux, approximately $1 \%$ of the average illuminance. The number of points outside of rounding error reduced with increased simulation settings; particularly for increased diffuse bounces and rays per point. The test case was therefore considered passed by both simulators.

\subsubsection{Test case 6.11: Light source symmetry}

\section{Test case description}

This test case is similar to the first six test cases in testing photometrically defined point lights; but instead of specific illuminance values it tests for symmetry in the illuminances on a grid. Three different light sources are tested, axially symmetric, quadrilateral and bilaterally symmetric, which are positioned in the centre of the ceiling of a four by four by three metre high room of zero reflectance. They illuminate a twenty by twenty point grid of measurement points on the floor, which are checked for the relevant symmetry.

\section{Modelling issues}

There were no issues related to modelling with this test case.

\section{Simulation issues}

There were no simulation parameter issues with this test case.

\section{Results}

The results for this test case showed that both 3ds Max Design and Radiance were able to reproduce light source symmetry for all three scenarios. 


\section{Test case description}

This test case assesses the ability of a simulator to manipulate a photometrically defined point light to face different directions. The test case uses a completely black four metre cubed room, with an axially symmetric point light at its centre. Each surface has a ten by ten grid of measurement points, and the analysis is repeated six times with the light source pointed at each grid. The illuminances from each of the six light meters directly facing the source should match.

\section{Modelling issues}

There were no issues related to modelling with this test case.

\section{Simulation issues}

There were no simulation parameter issues with this test case.

\section{Results}

The results for this test case showed that both 3ds Max Design and Radiance were able to correctly replicate the aiming of a light source along the three principle axis in both the positive and negative directions.

\subsubsection{Test case 6.13: Internal shadows (mask to artificial source)}

\section{Test case description}

This test case consists of a one metre square area light source in the centre of the ceiling of a four metre square, three metre high room of zero reflectance. A twenty by twenty grid of measurement points on the floor is used to calculate the average illuminance. The test case is run twice, with a shading surface placed ten millimetres in front of the light source that covers half of its area for the second run.

\section{Modelling issues}

There were no issues related to modelling with this test case.

\section{Simulation issues}

There were no simulation parameter issues with this test case.

\section{Results}

The results for this test case should show a 50\% decrease in average illuminance with the shadowing object present. Figure 49 and figure 50 below show the grid illuminances from both 3ds Max Design and Radiance respectively. The shadowing surface is present on the left side of the room in the right surface charts. 


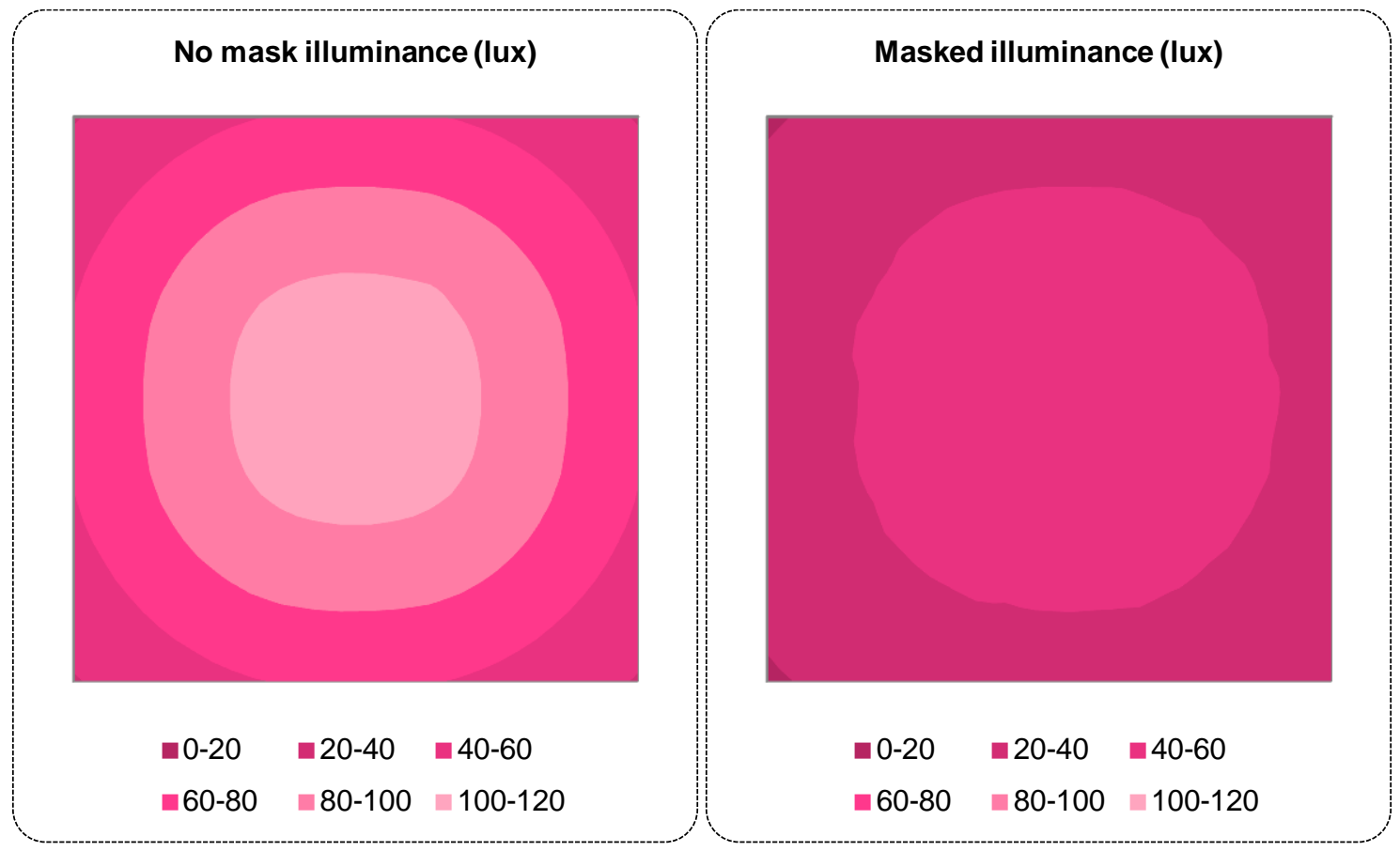

Figure 50: Test case 6.13 results for Radiance

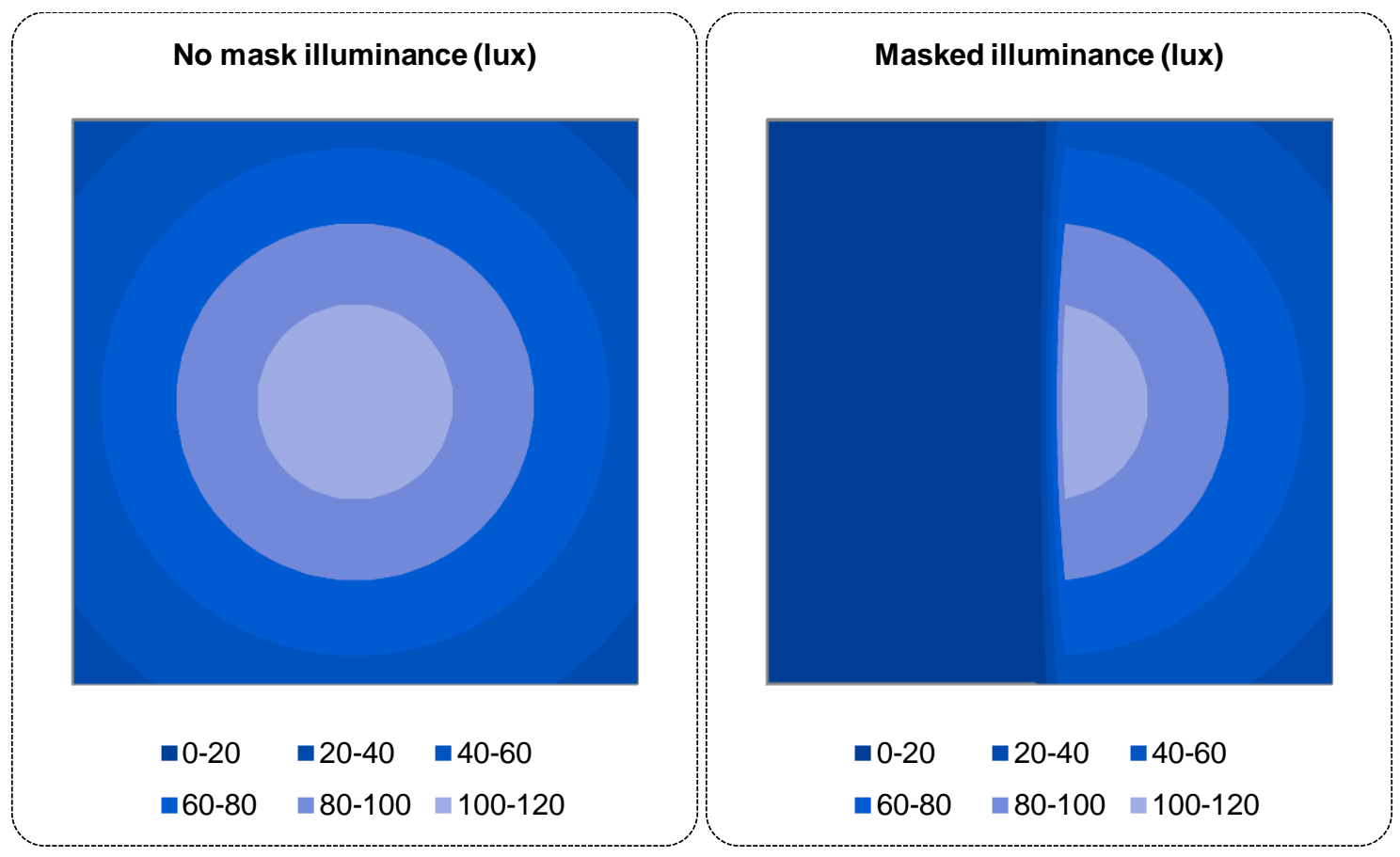

It can be seen that both simulators showed a reduction in illuminance - however with differing distributions. Both achieved the correct 50\% reduction in average illuminance. For $3 \mathrm{ds}$ Max Design, the distribution of light correctly moved more to the right but spread over all measurement points. For Radiance, half of the measurement points dropped to zero illuminance, with the other half remaining the same. This indicates Radiance is treating the area source as a point source. However, both simulators passed the test case based on the test case criteria. 
The validation of 3ds Max Design and Radiance against the CIE 171:2006 test cases has provided many conclusions; about the software, the test cases themselves, and the test cases' place in a potential daylight simulation validation dataset suite. These have been presented below in their own subsections. All of the test cases pertaining to daylighting that were deemed to be valid achieved a pass from both simulators, bar one; with 3ds Max design failing to simulate specular reflections.

\section{On the validation of $3 d s$ Max Design \& Radiance}

For the majority of the areas covered by the test cases, 3ds Max Design and Radiance performed similarly. From the test case results the following can be stated for both simulators:

- $\quad$ Point lights with IES LM-63-1995 file format photometric descriptions are simulated correctly.

- Point lights with uniform diffuse and isoradiant (uniform spherical) distributions are simulated correctly.

- $\quad$ Area lights, both with and without photometric descriptions are not correctly simulated. Radiance performs better than 3ds Max Design in this area.

- Both simulators are able to behave in a physically correct, luminous flux conserving fashion. However, without careful input, the laws of physics can be 'broken' by both simulators.

- Clear glass transmittances are correctly reproduced based on each simulator's chosen reflectivity model.

- Light reflection over diffuse surfaces is correctly handled, although care must be taken with the simulation parameters for number of rays traced in ray-tracing software. It is recommended that this value be increased when dealing with small, brightly lit surfaces.

- Based on the recalculated analytical values for test case 5.7 by Geisler-Moroder and Dür, obstructions to diffuse reflecting surfaces are simulated correctly.

- Both simulators correctly simulate the internal reflected component of a diffusely reflecting square room, although require much higher than standard diffuse or ambient bounce simulation parameters for average reflectances of $70 \%$ or above. This gives premise for increasing this parameter for simulations with large surface areas of highly reflective surfaces, for example white painted rooms.

- $\quad$ The sky components of the CIE Clear and Overcast general sky types are correctly simulated.

- The accuracy of external reflected components is not fully known, and cannot be accurately tested without new analytical values. Based on the sky component, diffuse reflection and obstruction test cases however, it can be reasonably assumed that these are being simulated correctly.

- Sun patches can be correctly produced.

- Both simulators correctly respect the spectral components of light in their illuminance calculations.

- Both simulators can correctly model the CIE Sunny and Overcast skies as well as the Perez all-weather model.

- Date, time and location inputs to alter the previously mentioned sky models are accepted and translated correctly.

- If correct modelling procedures are followed then both simulators are not prone to significant light leaks.

- Room surface symmetry is correctly simulated.

- Light source axial, quadrilateral and bilateral symmetry is correctly simulated.

- Light source aiming is correctly simulated.

- Masks to internal light sources are correctly considered in terms of room average illuminances.

At an individual simulator level; for 3ds Max Design 2012:

- $\quad$ The CIBSE photometric file format produces incorrect light distributions.

- Pure diffuse surfaces are more accurate than area lights; but cannot use photometry.

- To correctly simulate the surface reflectance of clear glass the 'custom curve' option must be used in the Arch \& Design material type, rather than the automatic 'by IOR' setting.

- Specular reflections are not taken into account by the lighting analysis tools, despite the ability to simulate them for images.

- There is an anomaly caused by light meter objects placed close to external surfaces which can cause light leaks, most likely due to interpolation. 
- The limitation on the maximum value for the ambient bounces simulation parameter can potentially cause simulation inaccuracies for rooms with an average reflectance over $70 \%$.

- Specular reflections are correctly simulated, however with some inconsistencies where the ray-tracer was unable to find the mirror-reflected light source.

- While correctly calculating the reduction in luminous flux from a masked light source, the change in light distribution from a masked light source is not correctly simulated.

The largest difference between the two simulators was in the simulation of specular reflections. Radiance was proven capable of fully calculating them, whereas 3ds Max Design can only create images of them. This is significant as specular reflecting elements are increasingly popular in daylight systems. However, despite the fact Radiance was able to simulate them; it is likely that the necessity to simulate specular reflections will reduce with the increase in use of BSDFs for complex daylight systems involving specular components.

Area lights are of particular concern for both simulators, but particularly for 3ds Max Design which required a non-standard modelling technique to achieve a similar result to Radiance. This is significant, as area lights are likely to make up a large portion of lighting in office electric lighting scenarios, the most likely scenarios to be simulated by designers. It is therefore recommended that the developers of both software packages examine their methods of calculating the light distribution from area light sources.

\section{On the CIE 171:2006 test cases}

For the test cases themselves, a number of conclusions can be made. Firstly, a number of the test cases contained errors that require acknowledgement:

- The analytical solution for test case 5.7 is incorrect, and the adoption of new reference values calculated by Geisler-Moroder and Dür in 2008 is recommended.

- Test case 5.8 should acknowledge the errors introduced by using a cube rather than a sphere.

- Test cases 5.11-5.14 require new analytical values or entirely new test case scenarios in order to be reasonably tested in common simulation software.

It is therefore recommended that an erratum be produced and published as soon as possible to correct these issues.

It is also recommended that a full test case description and analytical solution set - particularly for test cases 6.3-6.5, be produced. Currently, it is not possible to test all of the elements set out to be tested as part of the CIE 171:2006 document. This would also ensure that one validation against the test cases, such as this one, can be directly compared to another; such as the 2007 validation of the AGi32 radiosity simulator by Dau Design and Consulting Ltd.

Given the recent rise in use of BSDFs in daylight simulation, it could also be recommended that a test case be developed to test their implementation in simulation software. Such a test case could be designed similarly to that for the testing of area light sources with photometry; however with a window opening with a standard sky. A standardised BSDF data file would be provided for use in the validation.

\section{Implications on this study}

As it stands the CIE 171:2006 test cases fit well into the lower end of the complexity range. Once an erratum has been published, and with the addition of better defined test cases for more specific aspects of light simulation, their place as the primary testing procedure for lighting simulation software will be solidified. However, the test cases give little to no indication of real-data performance in daylight simulation. Hence, the inclusion of the next two datasets, intended to test the real data performance of daylight simulation software, is justified 

This chapter documents the results of an orthogonal validation of 3ds Max Design and Radiance using an experimental empirically measured dataset from the Lawrence Berkeley National Laboratory in Berkeley, California. The dataset, consisting of a simple, single-opening office under various sky conditions, represents a step in complexity above the basic tests performed under the CIE 171:2006 test cases examined in the previous chapter. A rectangular office with a single window is the least complex scenario likely to be simulated by designers in practice. The addition of blinds for some of the data adds further granularity in the increase in complexity. Furthermore, as the Perez all-weather sky model was developed using data from the same location, it is useful in minimising error arising from the sky model and emphasising errors from other sources in the modelling and simulation process.

The data has been presented in a variety of formats to highlight various properties of the dataset and results of the validation. The first format is as a series of "sparklines" - small line graphs depicting the errors for each of the illuminance sensors in the test cell - presented on the fold-out page 71, preceded by a key and information to support the reading of the results. The sparklines are split by simulator, and then grouped by sensor - for this dataset only the six workplane sensors have been shown here, with the remaining ceiling and wall sensors shown in the appendix. Inside each of the sensor groups are twelve sparklines. The six at the left are for an unshaded test cell, under a variety of weather conditions. At the top right of each group are the three days simulated with blinds present, tilted level with the horizon. The bottom right contains the three sparklines for the days where the blinds were tilted at 45 degrees. If a sensor went outside of its operational range, causing data to be clipped, it has been highlighted in red. Finally, each sparkline is accompanied by three numbers, which indicate the proportion of the data within $\pm 40 \%, 20 \%$ or $10 \%$ thresholds. It is important to note that data within thirty minutes of sunrise and sunset has not been used in these or any later calculations of Mean Bias Error.

Below the key and next to the example layout is the direct normal radiation data for each of the days used in the simulations. This has been shown as a green sparkline ranging from $0 \mathrm{Wh} / \mathrm{m}^{2}$ at the bottom of each cell to $950 \mathrm{Wh} / \mathrm{m}^{2}$ at the top. For all sparklines shown the left side of the cells equates to $8 \mathrm{am}$, and $6 \mathrm{pm}$ at the right. This is to give an indication of the length of time daylight is present, the weather conditions and frequency in change of cloud cover. Directly below this are two sets of red error sparklines for the simulated versus measured global horizontal illuminance; ranging from $-100 \%$ at the bottom of the cell, $0 \%$ at the centreline, to positive $100 \%$ at the top of each cell. The errors are calculated using equation 2 from page 31 of section3.7, and it is the average of these errors that make up the Mean Bias Error.

Using the global illuminance error sparklines, a number of observations can be made. The first is that for all weather conditions, both 3ds Max Design and Radiance's implementations of the Perez all-weather sky model are producing accurate global illuminances. Errors are slightly larger on December $29^{\text {th }}$, a particularly variable day. The results are also consistent with the previous findings of poor accuracy for low sun angles for the Perez sky. As the data approaches sunset, 3ds Max Design begins to overestimate illuminances, whereas Radiance begins to underestimate. It is interesting to note that at sunset $3 \mathrm{ds}$ Max Design continues to produce skies of increasing inaccuracy without providing any warnings to the user, whereas Radiance's Gendaylit program for producing Perez skies ceases to produce values, hence the errors dropping to $-100 \%$ near the end of the day in some cases. 


\begin{tabular}{|l|l|}
\hline \multicolumn{2}{|c|}{ Key } \\
\hline & Clear sky \\
\hline & Mixed sky \\
\hline & Cloudy sky \\
\hline & Direct normal radiation $\left(\mathrm{Wh} / \mathrm{m}^{2}\right)$ \\
\hline & Mean Bias Error $( \pm \%)$ \\
\hline & Sensor out of range (data discarded) \\
\hline $40 \%, 20 \%, 10 \%$ & Percentage MBE within $+/$ - $40 \%, 20 \%, 10 \%$ thresholds \\
\hline
\end{tabular}

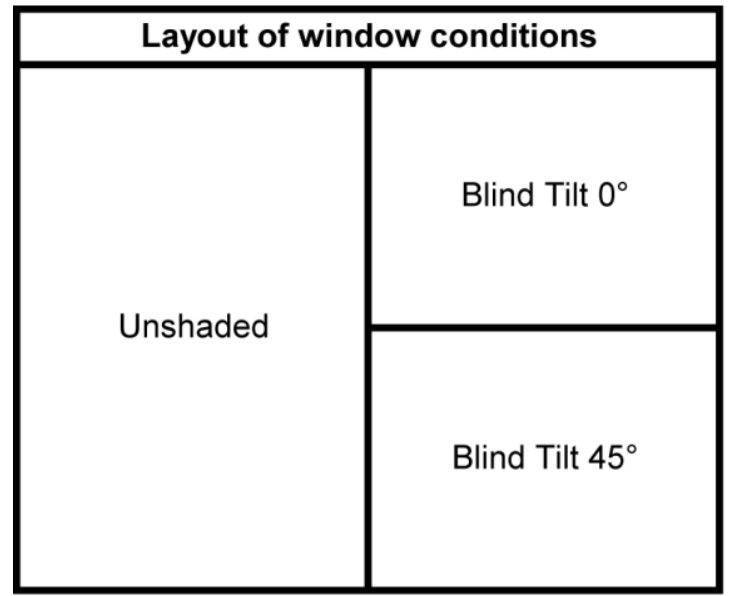

\begin{tabular}{|c|c|}
\hline Global illuminance check (3ds Max Design) \\
\hline & \\
\hline Jun 172010 & Jul 192010 \\
\hline Jan 152011 & Jul 202010 \\
\hline Jan 162011 & Jul 212010 \\
\hline Jun 182010 & Dec 302010 \\
\hline Jun 192010 & Dec 292010 \\
\hline Dec 4 2010 & Dec 28 2010 \\
\hline
\end{tabular}

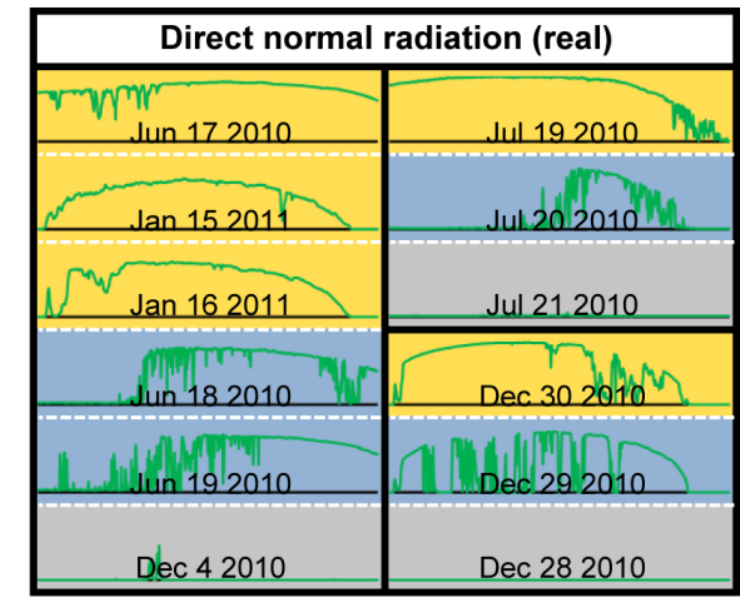

\begin{tabular}{|c|c|}
\hline \multicolumn{2}{|c|}{ Global illuminance check (Radiance) } \\
\hline & Jul 19 2010 \\
\hline Jun 17 2010 & \\
\hline & Jul 20 2010 \\
\hline Jan 15 2011 & \\
\hline Jan 16 2011 & Jul 21 2010 \\
\hline Jun 18 2010 & Dec 30 2010 \\
\hline Jun 19 2010 & Dec 29 2010 \\
\hline Dec 4 2010 】 & Dec 28 2010 \\
\hline
\end{tabular}


3ds Max Design 2012 (v14.0 SP2 Educational)

\begin{tabular}{|c|c|}
\hline \multicolumn{2}{|c|}{ Workplane Sensor 5} \\
\hline & \\
\hline $21.3 \%, 5.2 \%, 0.0 \%$ & $76.2 \%, 34.3 \%, 2.3 \%$ \\
\hline & \\
\hline $95.6 \%, 81.0 \%, 54.1 \%$ & $79.7 \%, 56.2 \%, 26.5 \%$ \\
\hline a & \\
\hline $96.6 \%, 66.3 \%, 41.0 \%$ & $91.2 \%, 82.8 \%, 47.0 \%$ \\
\hline $48.7 \%, 23.8 \%, 17.7 \%$ & $91.4 \%, 81.4 \%, 70.4 \%$ \\
\hline 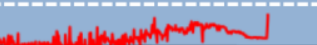 & Nallth, wh \\
\hline $57.5 \%, 37.3 \%, 26.3 \%$ & $72.0 \%, 52.5 \%, 40.6 \%$ \\
\hline 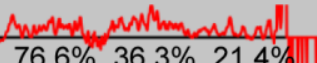 & $992 \% 841 \% 56.40$ \\
\hline & \\
\hline
\end{tabular}

\begin{tabular}{|c|c|}
\hline \multicolumn{2}{|c|}{ Workplane Sensor 3} \\
\hline then & \\
\hline $22.2 \%, 0.0 \%, 0.0 \%$ & $74.2 \%, 6.3 \%, 1.5 \%$ \\
\hline 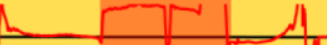 & \\
\hline $78.8 \%, 65.3 \%, 34.0 \%$ & $77.3 \%, 43.3 \%, 14.5 \%$ \\
\hline+ & \\
\hline $76.4 \%, 61.5 \%, 39.6 \%$ & $91.2 \%, 65.5 \%, 17.8 \%$ \\
\hline 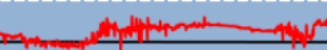 & \\
\hline $57.3 \%, 32.3 \%, 17.5 \%$ & $92.7 \%, 86.7 \%, 73.1 \%$ \\
\hline why when & 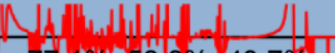 \\
\hline $62.3 \%, 44.2 \%, 31.8 \%$ & $77.1 \%, 58.8 \%, 43.7 \%$ \\
\hline 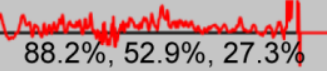 & in \\
\hline
\end{tabular}
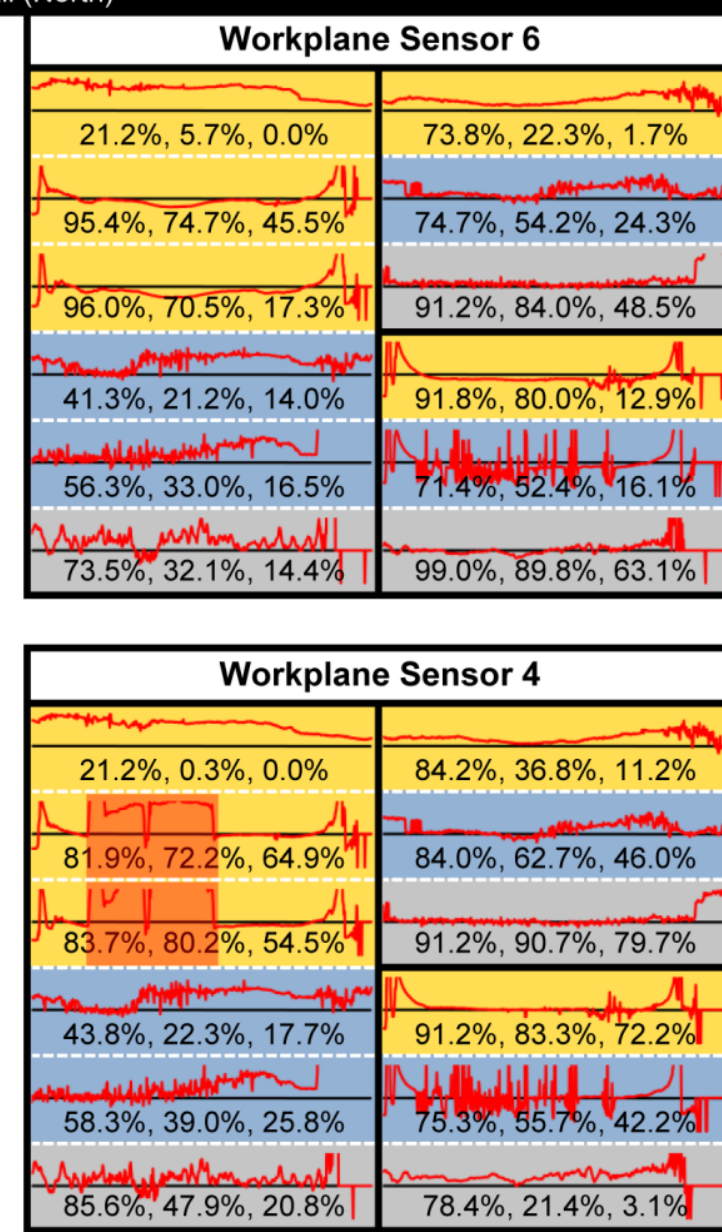

\begin{tabular}{|c|c|c|c|}
\hline \multicolumn{2}{|c|}{ Workplane Sensor 1} & \multicolumn{2}{|c|}{ Workplane Sensor 2} \\
\hline Whan & & Whan & \\
\hline $0.0 \%, 0.0 \%, 0.0 \%$ & $81.0 \%, 53.0 \%, 29.7 \%$ & $5.8 \%, 0.0 \%, 0.0 \%$ & $83.0 \%, 71.8 \%, 48.2$ \\
\hline$\pi N^{\prime}$ & & |WI & \\
\hline $79.8 \%, 29.8 \%, 6.0 \%$ & $91.3 \%, 67.5 \%, 52.7 \%$ & $56.0 \%, 27.4 \%, 9.5 \%$ & $94.2 \%, 75.3 \%, 50.0 \%$ \\
\hline$\sqrt{ }-7\left|\mathrm{~h} \mathrm{~N}^{\prime}\right|$ & & तो & \\
\hline $78.6 \%, 63.1 \%, 41.7 \%$ & $92.0 \%, 90.7 \%, 81.7 \%$ & $41.7 \%, 34.5 \%, 9.0 \%$ & $91.5 \%, 90.8 \%, 77.5 \%$ \\
\hline 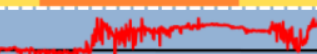 & I斤 & 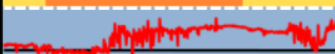 & \\
\hline $41.5 \%, 34.2 \%, 27.8 \%$ & $87.6 \%, 74.7 \%, 17.5 \%$ & $42.7 \%, 34.7 \%, 18.3 \%$ & $91.6 \%, 83.9 \%, 73.3 \%$ \\
\hline 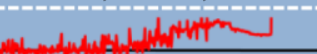 & IVA & 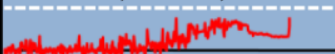 & 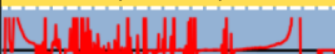 \\
\hline $51.3 \%, 41.2 \%, 29.7 \%$ & $73.7 \%, 52.9 \%, 13.5 \%$ & $51.5 \%, 41.5 \%, 30.7 \%$ & $75.7 \%, 56.1 \%, 43.7 \%$ \\
\hline 等 & & 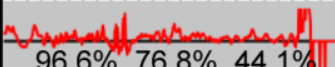 & \\
\hline $97.4 \%$ & $60.8 \%, 5.7 \%, 1.2^{c}$ & $6.8 \%, 44.1 \%$ & 78.0 \\
\hline
\end{tabular}

Radiance (v4.1)

\begin{tabular}{|c|c|}
\hline \multicolumn{2}{|c|}{$\overline{\text { ane } s}$} \\
\hline $69.0 \%, 14.7 \%, 9.0 \%$ & $99.5 \%, 78.2 \%, 43.2 \%$ \\
\hline $85.0 \%, 61.7 \%, 17.0 \%$ & $99.8 \%, 88.2 \%, 49.5 \%$ \\
\hline $81.9 \%, 30.3 \%, 0.4 \%$ & $98.8 \%, 91.5 \%, 62.5 \%$ \\
\hline & \\
\hline $97.3 \%, 41.3 \%, 22.2 \%$ & $55.5 \%, 5.5 \%, " 2: 9 \%$ \\
\hline 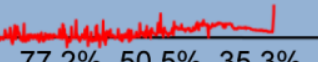 & 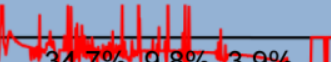 \\
\hline & \\
\hline $84.0 \%, 51.3 \%, 26.9 \%$ & $100.0 \%, 85.3 \%, 28.4 \%$ \\
\hline
\end{tabular}

\begin{tabular}{|c|c|}
\hline \multicolumn{2}{|c|}{ Workplane S€ } \\
\hline & \\
\hline $68.8 \%, 14.3 \%, 8.8 \%$ & $99.5 \%, 74.2 \%, 44.3$ \\
\hline $81.9 \%, 52.2 \%, 10.7 \%$ & $99.8 \%, 86.3 \%, 47.3 \%$ \\
\hline $81.7 \%, 23.8 \%, 5.5 \%$ & $98.7 \%, 92.0 \%, 62.3 \%$ \\
\hline & \\
\hline $96.5 \%, 38.0 \%, 22.8 \%$ & $44.3 \%, 4.9 \%, 2.5 \%$ \\
\hline 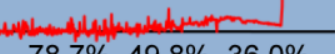 & thenth \\
\hline $78.7 \%, 49.8 \%, 36.0 \%$ & $27.1 \%, 9.0 \%, 3.5 \%$ \\
\hline 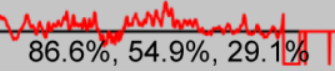 & $99.2 \%, 81.0 \%, 32.2 \%$ \\
\hline
\end{tabular}

\begin{tabular}{|c|c|}
\hline \multicolumn{2}{|c|}{ Workplane Sensor 3} \\
\hline & \\
\hline $74.7 \%, 14.3 \%, 7.5 \%$ & $99.7 \%, 81.5 \%, 28.2 \%$ \\
\hline H. & \\
\hline $55.9 \%, 37.8 \%, 16.7 \%$ & $100.0 \%, 87.5 \%, 54.8 \%$ \\
\hline $\mathbb{N}$ & \\
\hline $57.3 \%, 30.2 \%, 4.2 \%$ & $99.7 \%, 92.3 \%, 62.8 \%$ \\
\hline & \\
\hline $96.7 \%, 45.2 \%, 32.0 \%$ & $68.2 \%, 6.7 \%, 2.9 \%$ \\
\hline whthend & 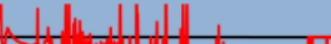 \\
\hline $78.5 \%, 51.5 \%, 31.8 \%$ & $49.4 \%, 43.7 \%, 5.1 \%$ \\
\hline $95.8 \%, 65.5$ & $100.0 \%, 95.3 \%, 67.3 \%$ \\
\hline
\end{tabular}

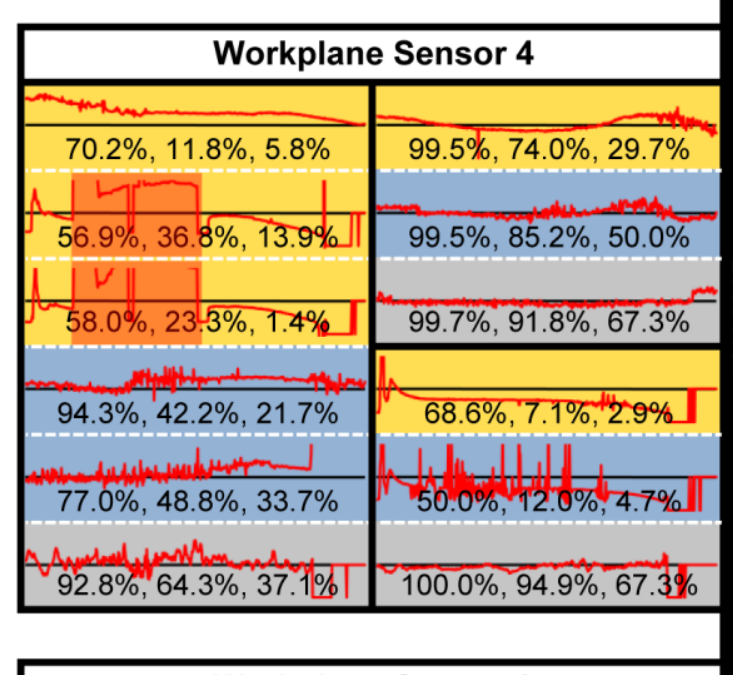

\begin{tabular}{|c|c|}
\hline \multicolumn{2}{|c|}{ Workplane Sensor 1 } \\
\hline $22.2 \%, 5.8 \%, 1.2 \%$ & $99.7 \%, 58.0 \%, 29.5 \%$ \\
\hline $79.8 \%, 29.8 \%, 6.0 \%$ & $100.0 \%, 79.7 \%, 37.2 \%$ \\
\hline $78.6 \%, 63.1 \%, 41.7 \%$ & $100.0 \%, 85.0 \%, 18.8 \%$ \\
\hline & \\
\hline $80.0 \%, 41.0 \%, 15.8 \%$ & $78.6 \%, 44.9 \%, 5.5 \%$ \\
\hline $73.3 \%, 44.5 \%, 22.3 \%$ & $59.0 \%, 19.6 \%, 8.8 \%$ \\
\hline $99.0 \%, 82.4 \%, 50.50 .3 \%$ & $99.2 \%, 94.5 \%, 64.7 \%$ \\
\hline
\end{tabular}

\begin{tabular}{|c|c|}
\hline \multicolumn{2}{|c|}{ Workplane Sensor 2} \\
\hline rashow & \\
\hline $62.0 \%, 8.3 \%, 3.3 \%$ & $99.5 \%, 59.2 \%, 29.3 \%$ \\
\hline Mir $\rightarrow$ & \\
\hline $56.0 \%, 27.4 \%, 9.5 \%$ & $99.8 \%, 71.7 \%, 30.7 \%$ \\
\hline $1 \mathrm{~d} \mathrm{w}^{\prime}$ & \\
\hline $41.7 \%, 34.5 \%, 49.0 \%$ & $100.0 \%, 73.5 \%, 7.5 \%$ \\
\hline & \\
\hline $94.7 \%, 45.8 \%, 30.7 \%$ & $74.1 \%, 7.5 \%, 2.5 \%$ \\
\hline 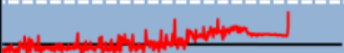 & 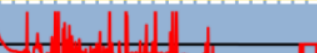 \\
\hline $75.8 \%, 49.0 \%, 23.7 \%$ & $53.1 \%, 12.7 \%, 6.3 \%$ \\
\hline (39.2\%, $83.0 \%, 52.9 \%$ & $100.0 \%, 97.8 \%, 81.4 \%$ \\
\hline
\end{tabular}



Also included in the results are the Mean Bias Errors (MBEs) for both 3ds Max Design and Radiance in the two tables below. These have been broken down by window condition (unshaded, with blinds, with tilted blinds) and sky condition (clear, mixed and cloudy). The bottom right-most value in the tables gives the overall MBE for each simulator for the LBNL 71T dataset. Each MBE in the totals has been weighted according to the number of points used to calculate it, which can be found in table 11 in the appendices.

Table 5: LBNL 71T workplane Mean Bias Errors for 3ds Max Design

\begin{tabular}{|l|l|l|l|l|}
\hline Condition & Clear sky & Mixed sky & Cloudy sky & Total \\
\hline Unshaded & $34.65 \%$ & $32.47 \%$ & $17.67 \%$ & $30.76 \%$ \\
\hline Blind tilt $\mathbf{0}^{\circ}$ & $26.56 \%$ & $18.24 \%$ & $13.48 \%$ & $19.42 \%$ \\
\hline Blind tilt $\mathbf{4 5}^{\circ}$ & $9.84 \%$ & $7.79 \%$ & $24.09 \%$ & $13.91 \%$ \\
\hline Total & $27.06 \%$ & $22.93 \%$ & $18.14 \%$ & $\mathbf{2 3 . 2 3 \%}$ \\
\hline
\end{tabular}

Table 6: LBNL 71T workplane Mean Bias Errors for Radiance

\begin{tabular}{|l|l|l|l|l|}
\hline Condition & Clear sky & Mixed sky & Cloudy sky & \multicolumn{1}{l|}{ Total } \\
\hline Unshaded & $-0.37 \%$ & $15.72 \%$ & $5.84 \%$ & $7.08 \%$ \\
\hline Blind tilt $\mathbf{0}^{\circ}$ & $-1.76 \%$ & $-3.05 \%$ & $-6.93 \%$ & $-3.92 \%$ \\
\hline Blind tilt $\mathbf{4 5}^{\circ}$ & $-32.24 \%$ & $-32.89 \%$ & $-5.86 \%$ & $-23.66 \%$ \\
\hline Total & $-7.78 \%$ & $-0.56 \%$ & $-2.62 \%$ & $-\mathbf{3 . 8 2 \%}$ \\
\hline
\end{tabular}

The validation results reveal a number of trends; firstly that over all of the data Radiance's illuminance predictions were significantly lower, and, on average closer to the measured data. This is despite the endeavour to keep the model geometry, materials, and simulation parameters equal. This means that the differences are likely within the simulators' algorithms. Crucially, Radiance achieved results well within the $\pm 20 \%$ accuracy limit set in section 3.7. Only for the tilted blind window condition did its MBEs fall outside of $\pm 20 \%$. This shows that for Radiance, the added complexity of the tilted blind may necessitate higher simulation parameters or alternative simulation techniques such as BSDFs. 3ds Max Design gave less over-estimation for the tilted blind condition. This is likely not indicative of better accuracy; but insufficient light gathering as with Radiance appearing as lower values moving the error closer to zero. This again advocates the use of higher simulation parameters for situations where a light source (in this case the sun and sky) is not directly visible to the measurement point.

It can also be seen, highlighted in figure 51 below, that both simulators are overestimating the clear sky June $17^{\text {th }}$ day for the unshaded window; with 3ds Max Design (dark pink line) overestimating by significantly larger portion than Radiance (dark blue line). For the two other clear days, both in winter (only January $15^{\text {th }}$ shown below, in the lighter pink and blue lines), the overestimation is much less and even goes to underestimation. This is either due to an anomaly in the data for the June days, or more likely an error introduced by the inaccurate modelling of the metallic mullion material. As the mullions are quite wide, a larger proportion of light is reflected off them in high sun angle summer situations than in winter where the sun is lower; striking more of the front (external) face of the mullion than the top, which acts almost as a small light shelf. 


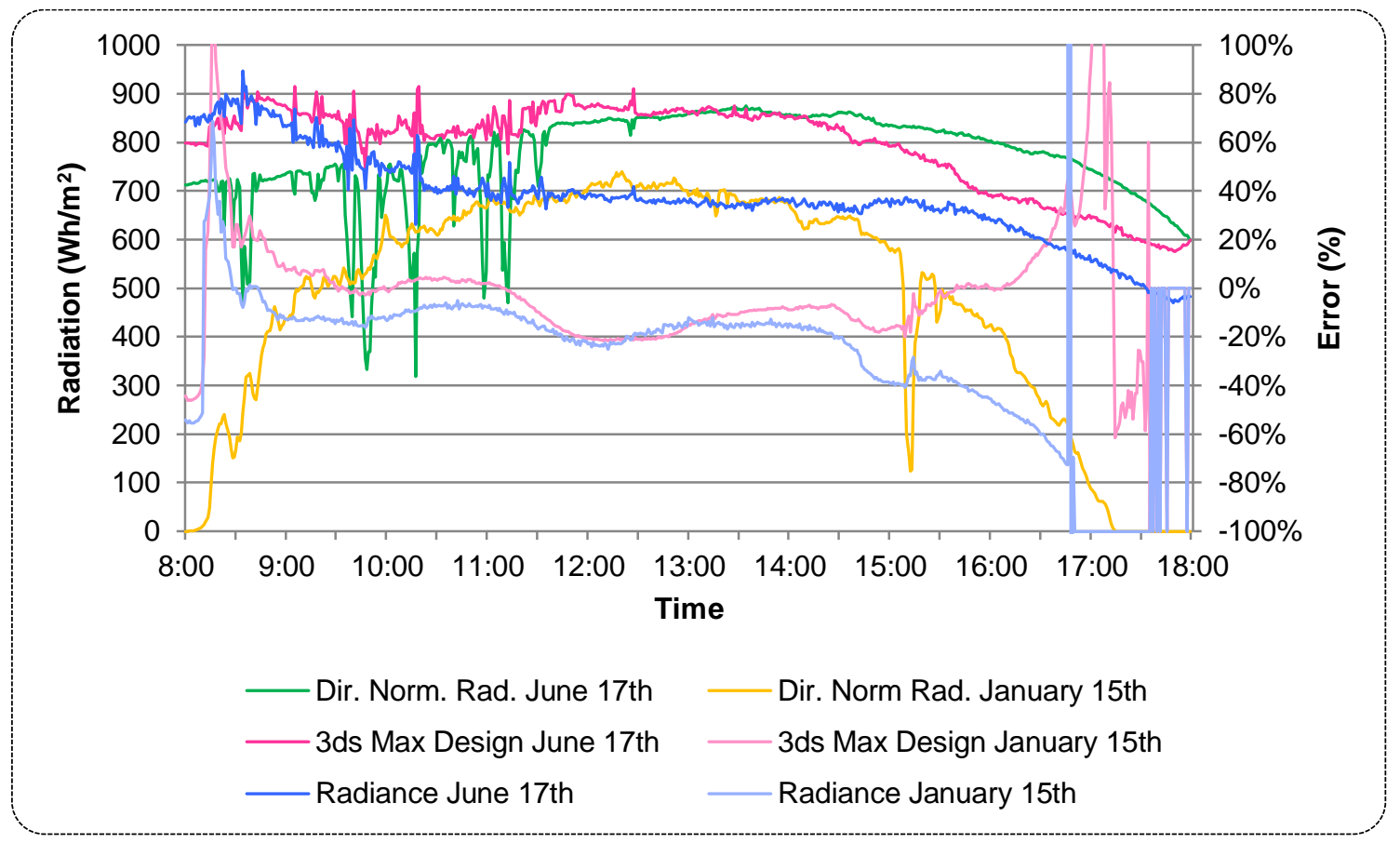

Figure 52: LBNL December 29 2010 direct normal radiation and workplane sensor 4 errors

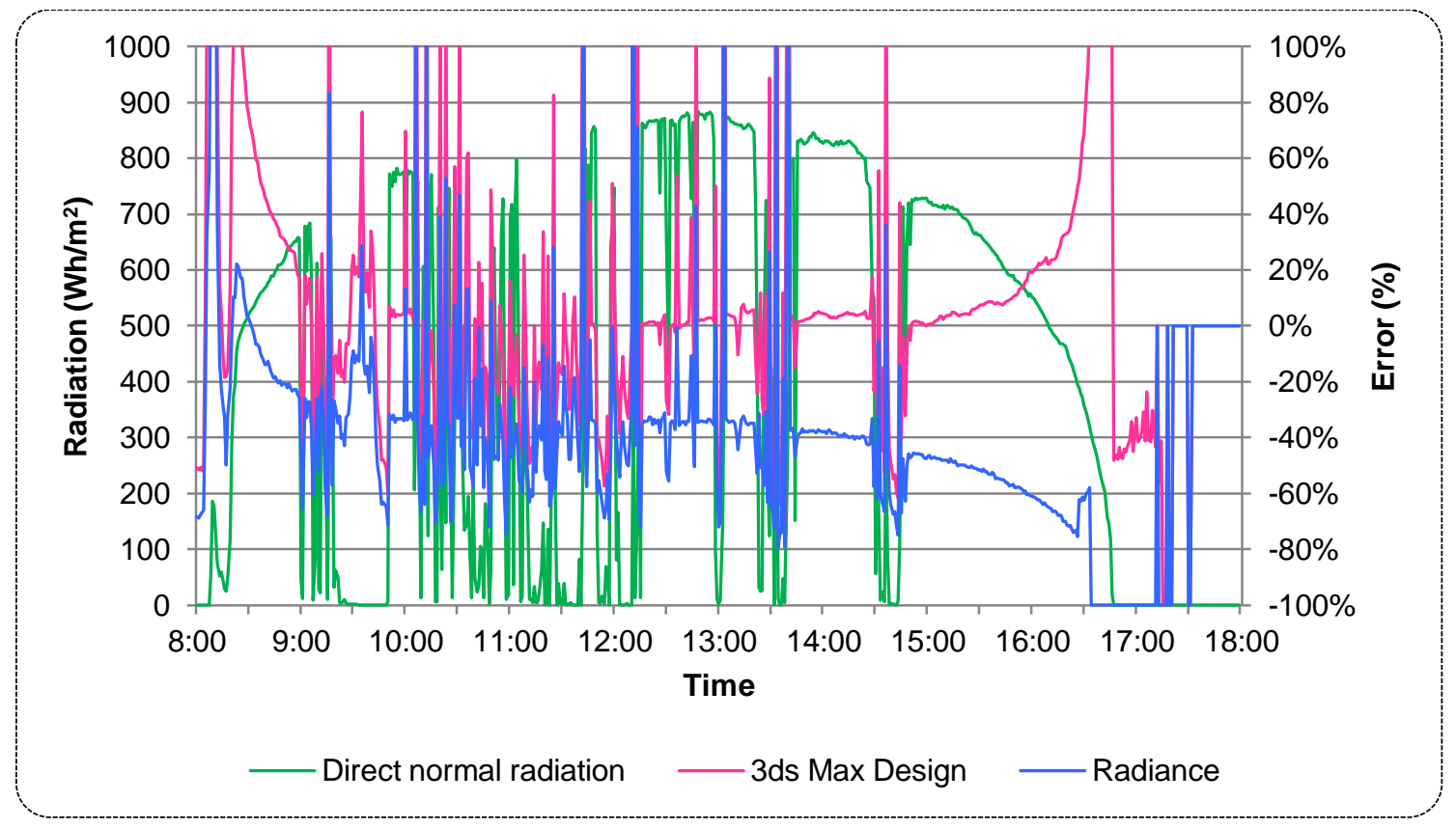

The days with mixed skies showed the most variability in error - as expected. The rapid change from sun to cloud and constant movement of cloud across the sky produced errors peaking both above and below $\pm 20 \%$ for both simulators; particularly visible for December $29^{\text {th }}$ as pictured for one workplane sensor in figure 52 above. However, these generally averaged over the 600 minutes of the day to produce MBEs consistent with the other sky types. This lends credence to the theory that inconsistencies introduced by the smoothing effect of mathematical sky models can be overcome with the use of many data points such is the case for both minute by minute simulations over a number of days and hourly simulations of an entire year. Figure 52 also shows the trend of errors increasing near sunrise and sunset; with 3ds Max Design overestimating for both and Radiance overestimating sunrise and underestimating at sunset.

There is a noteworthy lack of an expected trend for better accuracy at the front of the room (sensors 1 and 2) compared to the back (sensors 5 and 6). While it is present on some days it is missing on others, with no relationship to the window condition or sky type. This suggests some potential factors that could be at play; likely in combination: 
- The input geometry and material data for the test cell's interior is highly accurate, meaning there is very little compounding error once light has reached the inside. Most of the detected error is occurring at the barrier between the inside and outside; the ground plane, glazing and mullions.

- The ambient or diffuse bounce simulation parameter is high enough that most rays being traced successfully find a light source; even from the back of the room.

\section{Implications for designers}

Overall, the workplane illuminance predictions above were of better accuracy than those for the test cell's walls and ceiling sensors, the sparklines for which have been included as extra information in the appendices. This was expected, as the wall and ceiling sensors receive a higher proportion of light that has bounced several times rather than directly from the window. This allows for a greater amount of error to accumulate in the resulting illuminance predictions. Again this suggests that designers using simulations must consider how difficult it will be for the simulator to find a light source for the points they are calculating and adjust their simulation parameters accordingly.

The final finding of the validation was that despite the identical models and simulation parameters, 3ds Max Design was able to produce its illuminance predictions more quickly than Radiance. All simulations were run on a $2 x$ quad-core ( 8 total cores) $2.6 \mathrm{GHz}$ Intel Xeon machine running Microsoft Windows 7 , with $12 \mathrm{~GB}$ of RAM. For the full 600 minutes simulated per day, 3ds Max Design took approximately eleven minutes for the unshaded window condition models, and up to twenty minutes for the tilted blind condition. Radiance, scripted to run eight parallel rtrace instances (one per core), took approximately sixteen hours to complete one run of 600 minutes for the unshaded model and up to 48 hours for the tilted blind window condition. Eight rtrace instances were used in an effort to overcome the difference of 3ds Max Design natively using all cores available to it during simulation. Due to the 'black-box' nature of 3ds Max Design's Mental Ray light simulator, being closed rather than open-source as Radiance is, it is not known whether its time saving mechanisms for similar simulation parameters are what is causing the significant increase in error seen in this validation. The apparent trade-off in simulation time to precision is something designers must consider carefully, as there is some potential for different design decisions to be made depending on the choice of simulator.

\section{Implications for this study}

The results for this validation of 3ds Max Design and Radiance against the data collected from the Lawrence Berkeley National Laboratory's 71T test-bed show that the dataset is capable of being used in a successful validation. Any future validations should aim for at least equal if not better than the accuracy shown by the Radiance light simulator in this validation. The inclusion of the wall and ceiling data allows for further, more robust testing of a simulator that shows good accuracy for workplane illuminances. This supports the inclusion of the LBNL 71T test-bed dataset in any comprehensive daylight simulation validation dataset, as a good alternative to the BRE-IDMP dataset for simulators that cannot make use of its more complex skies. However, it does not satisfy the requirement of being complex 'real architecture', nor does it allow the testing of the Perez sky model outside of its 'home' climate, necessitating the inclusion of a further dataset. This is proposed to be a dataset recorded at the Université de La Réunion Laboratoire de Physique du Bâtiment et des Systèmes (LPBS), discussed in the next chapter. 

This chapter documents the results of a validation of 3ds Max Design and Radiance using an experimental empirically measured dataset from the Université de La Réunion's Laboratoire de Physique du Bâtiment et des Systèmes (LPBS) on the French colonial island of La Réunion. The dataset consists of two different buildings under various sky conditions. Again, like the LBNL 71T test-bed dataset, it represents a step in complexity above the basic tests performed under the CIE 171:2006 test cases examined in chapter 4. The dataset again includes a rectangular office with a single window as its first building scenario - the least complex scenario likely to be simulated by designers in practice - intended to enable comparisons with the LBNL dataset. This dataset also includes a classroom located in a specifically designed net-zero energy building at the LPBS. The classroom is open on two sides; to the North and South, via glass-louvered windows with a large proportion of mullion in each. The Southern windows are open to the surroundings, whereas the Northern windows open to an atrium with an open roof. Both sets of windows have timber louvres to exclude direct sunlight. This offers a step in complexity up from the LBNL dataset.

The data has again been presented in a variety of formats to highlight various properties of the dataset and results of the validation. Like the LBNL dataset, a series of "sparklines" - small line graphs depicting the errors for each of the illuminance sensors have been used. These have been presented on the fold-out page 79 , preceded by a key and information to support the reading of the results. The sparklines are again split by simulator, with 3ds Max Design on the left and Radiance on the right, and then grouped by building and sensor. All of the sensors for this dataset have been shown, as only two days of data were collected for both buildings. As both buildings have shading which excludes direct sunlight, no data clipping from sensors going out of range was present. Each sparkline is accompanied by three numbers, which indicate the proportion of the data within $\pm 40 \%, 20 \%$ or $10 \%$ thresholds. It is important to note that data within thirty minutes of sunrise and sunset has not been used in these or any later calculations of Mean Bias Error.

Below the key are the direct normal radiation data and error sparklines for both of the buildings. The direct normal radiation is again shown in green, ranging from $0 \mathrm{Wh} / \mathrm{m}^{2}$ at the bottom of each cell to $950 \mathrm{Wh} / \mathrm{m}^{2}$ at the top. For all sparklines shown the left side of the cells equates to $8 \mathrm{am}$, and $6 \mathrm{pm}$ at the right. This is to give an indication of the length of time daylight is present, the weather conditions and frequency in change of cloud cover. For the LBNL dataset the red error sparklines showed the difference between simulated and measured global horizontal illuminance; however for this dataset no outdoor illuminance measurements were available. The sparklines therefore show the difference between the simulated and 'measured' global horizontal illuminance calculated from the measured global horizontal radiation using luminous efficiency coefficients. These were determined by whether it was sunny or not, by the following conditions. If the direct normal radiation was greater than three times the diffuse horizontal $100 \mathrm{Im} / \mathrm{W}$, the average for the (dominant) sun was used. For all other times the global average of $115 \mathrm{~lm} / \mathrm{W}$ was used (Hopkinson, Petherbridge, \& Longmore, 1966, p. 51). This shows as some stepping artefacts in the sparklines, but give a good enough indication that the Perez all-weather sky model is behaving correctly for both simulators in the tropical location of La Réunion. The sparklines again range from $-100 \%$ at the bottom of the cell, $0 \%$ at the centreline, to positive $100 \%$ at the top of each cell. The errors are calculated using equation 2 from page 31 of section 3.7, and it is the average of these errors that make up the Mean Bias Error.

Using the global illuminance error sparklines, similar observations can be made to those of the LBNL dataset. Again, for the weather conditions provided, both 3ds Max Design and Radiance's implementations of the Perez all-weather sky model produce accurate global illuminances with no unexpected variation. The data reconfirms 3ds Max Design's tendency to overestimate illuminances towards sunset whereas Radiance ceases to continue producing sky descriptions. 


\subsection{Université de La Réunion LPBS result information \& key}

\begin{tabular}{|l|l|}
\hline & Clear sky \\
\hline & Mixed sky \\
\hline & Cloudy sky \\
\hline & Direct normal radiation $\left(\mathrm{Wh} / \mathrm{m}^{2}\right)$ \\
\hline & Mean Bias Error ( $\pm \%)$ \\
\hline & Sensor out of range (data discarded) \\
\hline & Percentage MBE within $+/-40 \%, 20 \%, 10 \%$ thresholds \\
\hline
\end{tabular}

\section{Test cell (simple office)}

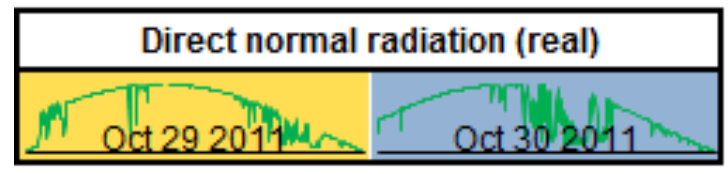

\begin{tabular}{|c|}
\hline Global illuminance check (3ds Max Design) \\
\hline Oct 292011 \\
\hline
\end{tabular}

\begin{tabular}{|c|}
\hline Global illuminance check (Radiance) \\
\hline Oct $292014^{4}-1$ \\
\hline Oct $302011^{2}-1$ \\
\hline
\end{tabular}

\section{Classroom (complex)}

\begin{tabular}{|c|}
\hline \multicolumn{2}{|c|}{ Direct normal radiation (real) } \\
\hline Aug 252011 \\
\hline
\end{tabular}

\begin{tabular}{|c|}
\hline Global illuminance check (3ds Max Design) \\
\hline \\
\hline Aug 252041 \\
\hline Aug 262011 \\
\hline
\end{tabular}

\begin{tabular}{|c|c|}
\hline Global illuminanc & check (Radiance) \\
\hline $\bar{A} u g 25204 \uparrow \longrightarrow$ & Aug 26201 \\
\hline
\end{tabular}


Test cell (simple office)

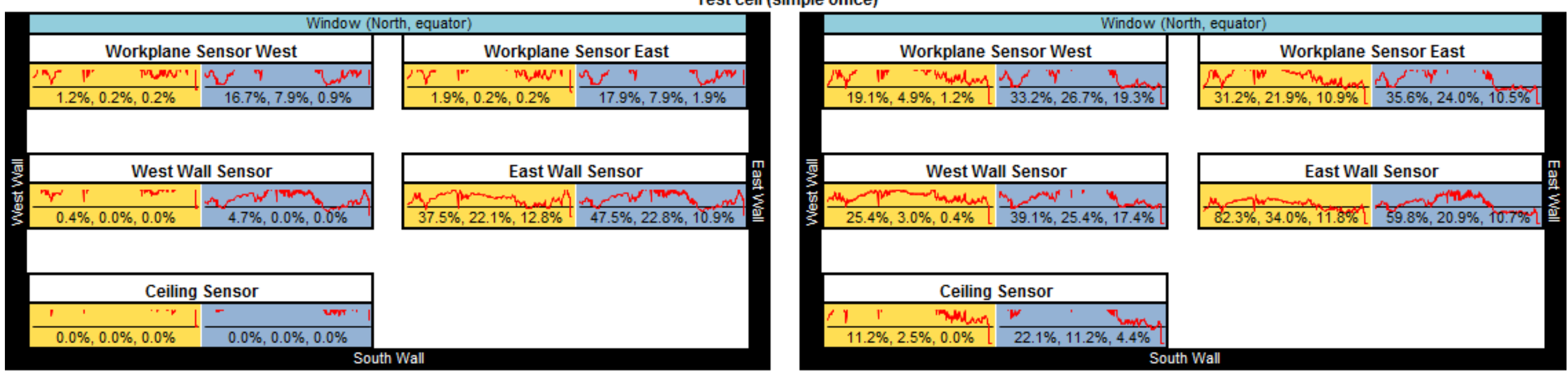

Classroom (complex)
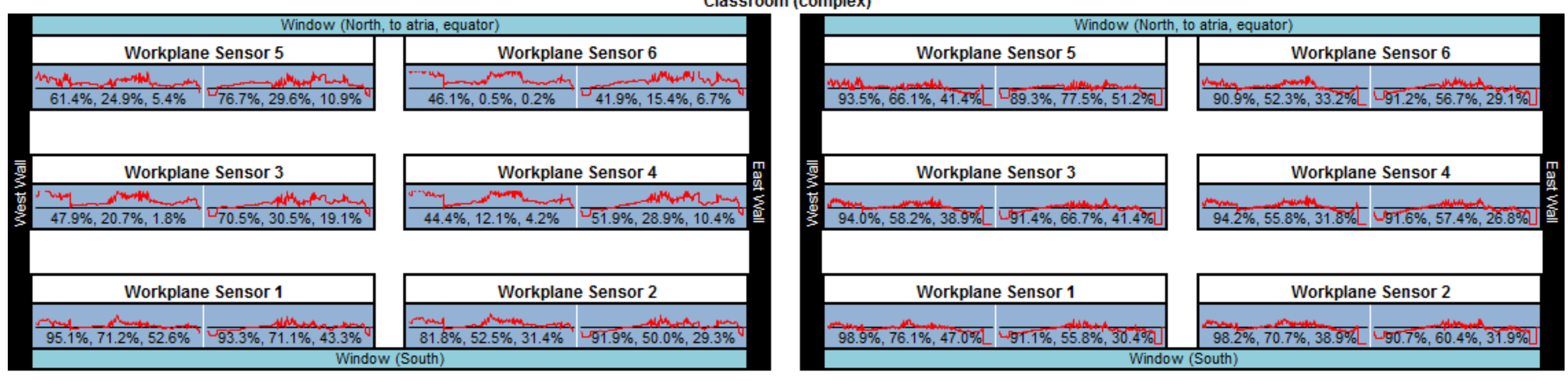
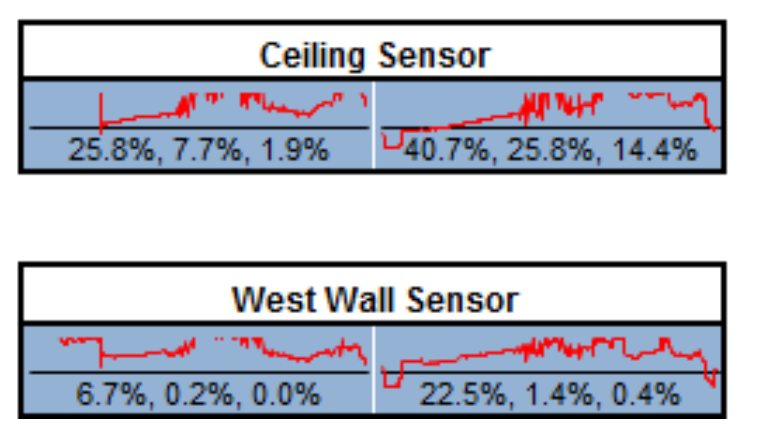

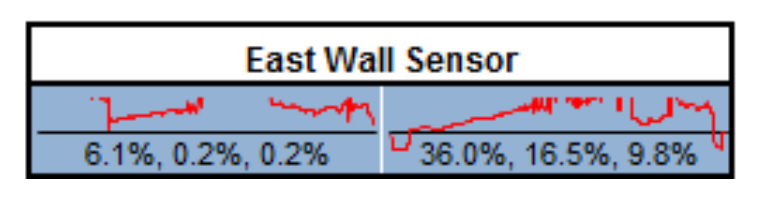

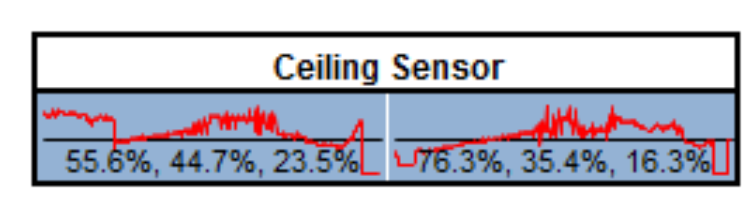
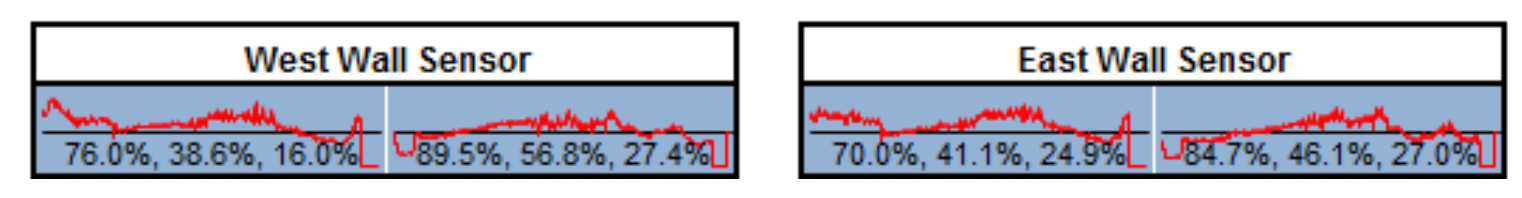

Jake Osborne 

Again included in the results are the Mean Bias Errors (MBEs) for both 3ds Max Design and Radiance in the two tables below. These have been broken down by scenario (simple office, complex classroom) and by date and sky condition (clear and mixed) as only two days of data were able to be obtained for each scenario. The right-most values in the tables give the overall MBE for each simulator for the two scenarios in the LPBS dataset. Each of the days has an equal number of data points so no weighting of averages was required.

Table 7: LPBS workplane Mean Bias Errors for 3ds Max Design

\begin{tabular}{|l|l|l|l|}
\hline Scenario & \multicolumn{2}{l|}{ Date/Weather } & Total \\
\hline \multirow{2}{*}{ Office (Simple) } & Oct 29 2011 & Oct 30 2011 & - \\
\cline { 2 - 4 } & $113.98 \%$ & $116.33 \%$ & $\mathbf{1 1 5 . 1 5 \%}$ \\
\hline Classroom (Complex) & Aug 25 2011 & Aug 26 2011 & - \\
\cline { 2 - 4 } & $38.17 \%$ & $20.36 \%$ & $\mathbf{2 9 . 2 7 \%}$ \\
\hline
\end{tabular}

Table 8: LPBS workplane Mean Bias Errors for Radiance

\begin{tabular}{|l|l|l|l|}
\hline Scenario & Date/Weather & \multicolumn{2}{l|}{ Total } \\
\hline \multirow{2}{*}{ Office (Simple) } & Oct 29 2011 & Oct 302011 & - \\
\cline { 2 - 4 } & $76.06 \%$ & $81.68 \%$ & $\mathbf{7 8 . 8 7 \%}$ \\
\hline Classroom (Complex) & Aug 25 2011 & Aug 26 2011 & - \\
\cline { 2 - 4 } & $4.10 \%$ & $-8.74 \%$ & $\mathbf{- 2 . 3 2 \%}$ \\
\hline
\end{tabular}

The validation results show a number of important trends. The first and most important is the extremely large error recorded for the Office data, which is much too large to be simulator error given the consistent performance of both simulators and their abilities to predict illuminances in the previous two datasets. It is thought that a large proportion of this error comes from the material properties of the room; which had many posters on its wall significantly reducing its average reflectance. This, along with an un-modelled obstructing building approximately fifty meters from the office, potentially accounts for the majority of the overestimation of illuminances. Test simulations of $50 \%$ lower reflectance on two walls showed a significant reduction in errors, producing MBEs for 3ds Max at approximately $40 \%$, which, when extrapolated to Radiance would equate to approximately $\pm 10 \%$. However, without better measured data, the results from the office data cannot be trusted.

The differences in illuminance predictions between 3ds Max Design and Radiance for both buildings in this dataset are consistent with those found in the LBNL dataset. Again, as significant care was taken to ensure model geometry, materials and simulation parameters equal, the differences are likely coming from the simulators' algorithms. For the classroom Radiance achieved results well within the $\pm 20 \%$ accuracy limit set in section 3.7. 3ds Max Design fell outside of the limit for both days simulated. The results are however of comparable accuracy to the LBNL dataset, suggesting that both simulators coped equally well with the added complexity. 
Figure 53: LPBS August $25^{\text {th }} 2011$ direct normal radiation and workplane sensor 1 errors

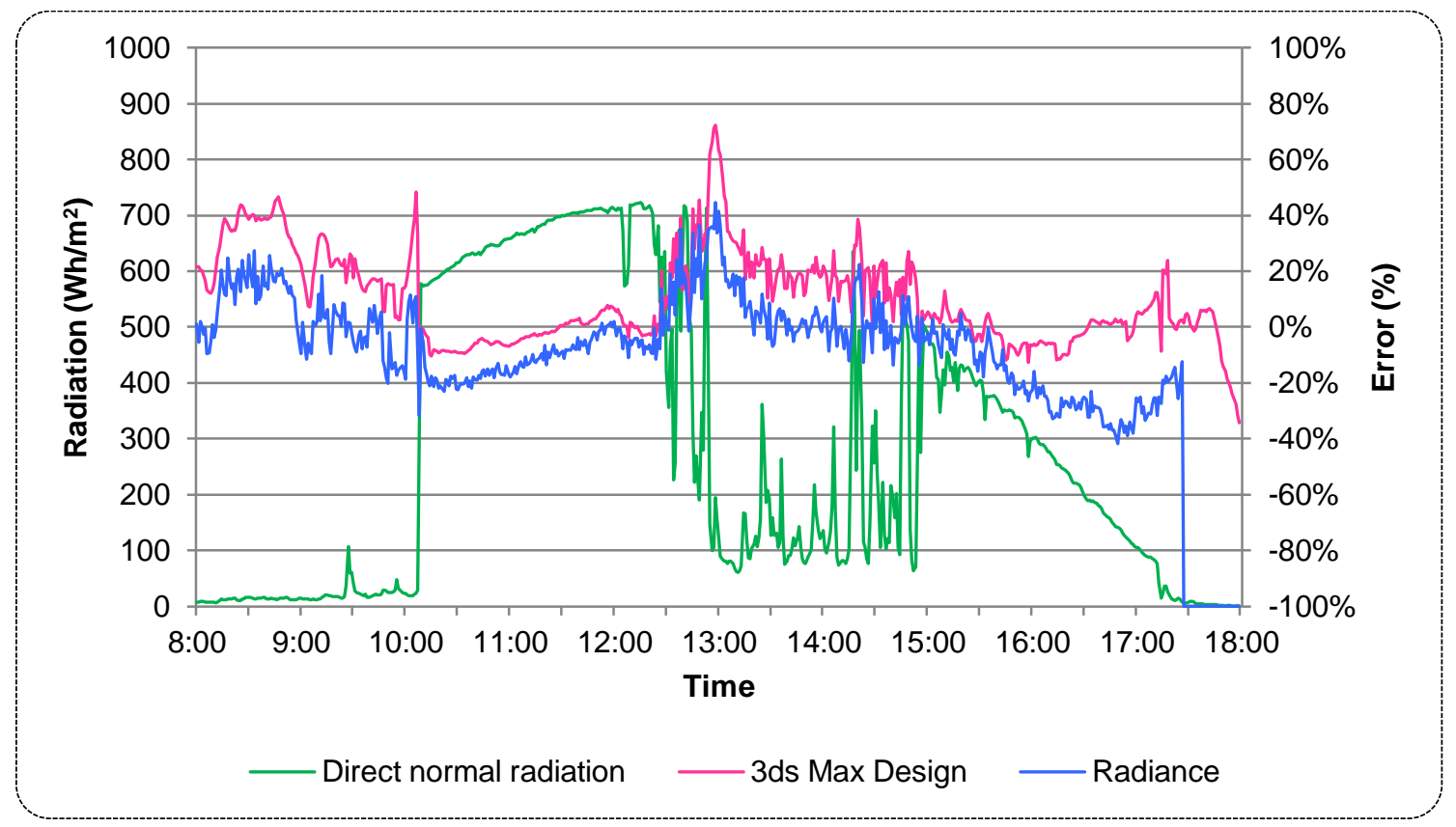

Again the days with mixed skies showed high variability in error as expected. The data for August $25^{\text {th }}$ in figure 53 above shows the peaks in error around the transition from direct sunlight to cloud cover, and overall higher errors during the partial cloud cover times in the morning and afternoon. Again these generally averaged out over the day to produce representative MBEs; although the effect is not as visible here as in the LBNL dataset. The theory that inconsistencies introduced by the smoothing effect of mathematical sky models can be overcome with the use of many data points has not been disproven.

Looking again at the sparklines, there is a clear trend of better accuracy for workplane sensors one and two of the classroom; closest to the Southern windows. These sensor points have the 'easiest' path to the light sources; the sun and sky. Errors increase towards the more complex, atrium-lit North side of the room. This suggests that the simulation parameters may not be high enough to successfully navigate the long light path from the interior through the shading elements and to the light source.

\section{Implications for designers}

With the simulators both showing error above the level that could have an effect on design, the relatively high simulation parameters used for this validation could be considered a minimum starting point for simulations of complex buildings, and could even be revised higher. Sensitivity testing of simulation parameters on a per-project basis is again seen as highly recommended. As with the LBNL dataset, the workplane illuminance predictions for the classroom were of better accuracy than those for classroom's wall and ceiling sensors. As before this was expected, as the wall and ceiling sensors receive a higher proportion of light that has bounced several times rather than directly from the window allowing for a greater amount of error to accumulate in the resulting illuminance predictions. Again this suggests that designers using simulations must carefully consider how difficult it will be for the simulator to find a light source for the points they are calculating and adjust their simulation parameters accordingly.

As with the LBNL dataset, 3ds Max Design was able to produce its illuminance predictions more quickly than Radiance. All simulations were again run on a 2x quad-core (8 total cores) $2.6 \mathrm{GHz}$ Intel Xeon machine running Microsoft Windows 7, with 12GB of RAM. For the full 600 minutes simulated per day, 3ds Max Design took approximately sixteen minutes for the office model, and approximately two hours for the classroom. Radiance, scripted to run eight parallel rtrace instances (one per core), took approximately 24 hours to complete one run of 600 minutes for the office, and approximately three days for the classroom. Eight rtrace instances were used in an effort to overcome the difference of 3ds Max Design natively using all cores available to it during simulation. Due to the 'black-box' nature of 3ds Max Design's Mental Ray light simulator, being closed rather than open-source as Radiance is, it is not known whether its time saving mechanisms for similar simulation parameters are what is causing the significant increase in error seen in this validation. The apparent trade-off in simulation time to accuracy is something designers must consider and balance carefully. 


\section{Implications for this study}

The results for this validation of 3ds Max Design and Radiance against the data collected from the Université de La Réunion's Laboratoire de Physique du Bâtiment et des Systèmes show that the dataset is suitable for usage in a daylight simulation validation study. Any future validations should aim for at least equal if not better than the accuracy shown by the Radiance light simulator in this validation. The increase in Mean Bias Error over the LBNL dataset suggests that the LPBS classroom scenario is close to the highest complexity that the software can successfully simulate using standard techniques. This makes it ideal for inclusion in a daylight simulation validation dataset suite, as a final step prior to testing more advanced techniques dealing with complexity such as BSDFs and HDRI skies. As it stands, the office data is not of sufficient quality to warrant its presence. The inclusion of the wall and ceiling data allows for further, more robust testing of a simulator that shows good accuracy for workplane illuminances. This supports the inclusion of the LPBS classroom dataset in any comprehensive daylight simulation validation dataset, as a good accompaniment to either the BRE-IDMP dataset or the LBNL dataset for simulators that cannot make use of its more complex skies. It fully satisfies the requirement of being complex 'real architecture', and, with the inclusion of better office data allows the testing of the Perez sky model outside of its 'home' climate. 

The primary aim of this research was to produce a suite of highly documented datasets for use in daylight simulation validation studies; that cover a range of complexities from simple tests to complex real data. This aim has been met by the inclusion of three datasets in a proposed suite; consisting of the CIE 171:2006 Test Cases to Assess the Accuracy of Lighting Computer Programs (basic), data from the 71T test-bed at Lawrence Berkeley National Laboratory (simple), and data from the Université de La Réunion's Laboratoire de Physique du Bâtiment et des Systèmes (complex). The suite - like the IES's BESTEST suite for energy simulators - covers a good range of simulation scenarios likely to be encountered by designers in practice. The suite fills a gap for validation data usable by daylight simulators unable to natively model patch-based skies. It is not currently able to test sky model accuracy for multiple locations, although this will be possible with some future work.

The dataset suite has been tested and shown to be highly successful in validation of $3 \mathrm{ds}$ Max Design and Radiance. The general agreement between the two simulators suggests the data is of high quality. It has provided high-quality validation results from most of its constituent data, with clear findings of both of the simulators' strengths and limitations. The dataset suite will enable other daylight simulation software packages to be successfully validated and compared to the results provided here. It is recommended that any new or updated daylight simulator be validated first against the CIE 171:2006 test cases. In addition, if it is able, it should be validated against the BRE-IDMP dataset. If the simulator cannot use the patch-based skies, or is more likely to be used with simpler weather data such as which is currently widely available through the US-DOE in conjunction with the Perez all-weather sky model, it should be validated using the LBNL 71T test-bed for simple scenarios and the LPBS classroom for more complex scenarios.

The validation process also produced a number of conclusions dealing with the Perez sky model. With regard to the validation dataset, it showed that there is potential for massive error due to the smoothing of a discontinuous real sky across the continuous mathematical function of the sky model. However, it was shown that when using many time steps, as with minute by minute data for a day or hourly data for a year these errors are likely to cancel out and give an accurate representation of real sky conditions.

\subsection{Lessons for designers}

The results of the validation have provided useful information for designers on both 3ds Max Design and Radiance simulators. They have also provided information for designers on the ways in which daylight programs should be used in day to day consultancy. It is recommended that users of simulation software pay significant attention to the parameters they are using and their appropriateness for different tasks.

\subsubsection{Accuracy}

The validation has shown that Radiance generally had better illuminance prediction precision, but with a significant time penalty over $3 \mathrm{ds}$ Max Design. This suggests that $3 \mathrm{ds}$ Max Design may be better suited to quick, iterative early sketch designs, and Radiance better suited for the final, complete building simulations involving smaller, more detailed changes. Neither program was inaccurate to the point of producing misleading results. Design trends were the same for both. However, Radiance more often produced a number that was more precisely matched to the measured data.

\subsubsection{Simulation parameters}

The validation process has also reinforced the validity of the simulation parameters used in this study as a starting point for designers conducting their own daylight simulations. These are similar to those first proposed by Reinhart in his 2009 validation of 3ds Max Design and Daysim. The results of the CIE test cases suggest that for rooms with an average reflectance over $70 \%$, higher ambient bounce simulation parameters are needed than even the high numbers suggested in these validations. In the special case of a room with all surfaces with a $95 \%$ reflectance, the ambient bounces required reach a disproportionately high figure of 128-plus. This is difficult with Radiance which becomes increasingly limited in its rays cast beyond eight bounces.

The results also showed that for situations involving small surface areas reflecting a large proportion of the light in a scene, the number of rays traced should be increased. 
The LBNL simple office scenario suggested that these simulation parameters could in fact be lower for such simple daylighting scenarios, and the LPBS classroom validation data suggested that the given simulation parameters were at the lower limit for what is needed for a complex building. Because these simulation parameters have more effect on the overall accuracy of the outcome than the inherent prediction capability of the individual programs, simulators deviate from them at their peril. These parameters heavily influence the speed of a simulation. The only general guidance that can be offered to speeding up simulations by reducing the settings to gain simulation speed is that each set of reduced parameter simulations should be calibrated against a full parameter simulation.

\subsubsection{Modelling parameters}

Designers must also take particular care in a number of areas that may not be obvious at first, as discovered in the chapter on quality assurance. Particularly, the size of a room being just as if not more important than the materials within it was unexpected. The amount of sensitivity to the geometric dimensions of a space and surroundings as well as the material properties is significant, and designers should use values as close as possible to those expected to be used in the final design of a building. Even the general shape of mullions in windows should be modelled. The maintenance factor of glazing has been shown to be more important than the glazing properties themselves in some scenarios, with potential for massive illuminance reductions for dirty windows. This means designers may need to specify not only an appropriate glass type, but a cleaning regime to ensure adequate indoor illumination.

\subsubsection{Sky models}

It was confirmed that for low sun angles, the Perez sky model has a tendency to increase in error; suggesting that designers not use illuminance simulations of daylight within a half hour of sunrise or sunset. It was also shown that for the Perez sky model the values for sky clearness - in this case defined by the dew point - have a higher sensitivity to error than the radiation values themselves. The lesson for designers from this is to ensure that the weather file for the location has reliable and representative data for solar radiation and for humidity.

\subsection{Lessons for software developers}

It is recommended that all lighting simulation software developers examine and improve their documentation on simulation parameters - to be clearer on what situations the defaults are to be used for and how to adjust them to be appropriate to different lighting scenarios. This must be informed by validation by a dataset such as the suite proposed by this study. 
Within the scope of this project, not all issues with the data and the programs that were identified could be resolved. A number remain for future research and development projects:

\section{On the CIE 171:2006 test cases}

The first step of future work on this project is to aid in producing an erratum for the CIE 171:2006 test cases, updating or replacing those cases for which the analytical values are invalid. This is especially important for test cases 5.11 through 5.14, involving skies, external reflections and obstructions, due to their significance to daylight simulation. In addition it is recommended that the set of analytical test cases numbered from 6.1 through 6.9 be given more specific test case descriptions and analytical values, to enable better comparison between simulators; one of the original goals of the test cases.

\section{On the Université de La Réunion LPBS dataset}

It is important that higher quality simple office data for the LPBS dataset be acquired for comparison against the simple office of the LBNL dataset. A better-suited office space; unobstructed and free of complicated wall coverings should be sought. Without this data, the validation dataset comprises the CIE 171:2006 test cases, the LBNL 71T test cell data, and the LPBS complex building data. This forms a robust complex light path validation dataset. Addition of a more reliable simple office or test cell data set to the suite would enable software developers to examine the differences between locations that their sky models can cope with. In this regard, the data from Canada used in the 3DS Max Design and Daysim validation (Reinhart, 2009) would make a useful addition to this suite.

It would also be beneficial to obtain more data for the classroom, for a wider variety of weather conditions and for both high and low sun angles in the summer and winter. This would allow for a more robust assessment of the simulation software's ability to navigate the timber louvres present on the building.

\section{On the proposed dataset suite as a whole}

The proposed dataset suite has been shown to provide a robust and unified set of validation tools for daylight simulation software. But, like any toolbox, there is always potential for future additions. Datasets containing different sources of complexity that still require or can be simulated by traditional techniques such as specular light shelves, unable to be approximated by BSDF - are an obvious choice for inclusion. Translucent window panels are another. The suite could also include more locations; especially ones with persistent, but uncommon weather features which could potentially highlight the shortcomings of the current stock of mathematically derived sky models.

The dataset suite should also look to include newer simulation techniques, like the BSDF, as part of its testing. Given that the LBNL is currently developing rigorous BSDF measurement and testing methods, they are a potential source. Having simultaneous control data for the parallel testing of standard simulation techniques is another positive factor in their favour. There is also potential for testing of short time step HDRI skies in parallel with the simple weather data similar to that already used in the LBNL dataset in this study, should the necessary measurement equipment be installed at the facility. This could provide an elegant single dataset to test many aspects of daylight simulation at once - from the simple unshaded office and one with complex shading systems as have been tested here but more broad, to even more complex systems requiring BSDFs, and finally testing the accuracy and practicality of HDRI skies.

Should HDRI sky implementation - popular for architectural visualisations - prove valuable to daylight simulation there is also the opportunity to 'convert' the existing, high quality patch-based skies of the BRE-IDMP dataset to HDRI images. This could allow for testing against the current "gold standard" dataset, in addition to HDRI sky implementation in simulation software.

\section{For the software developers}

Autodesk, the developers of 3ds Max Design, and Mental Images, developers of the Mental Ray raytracing engine, used in the pilot orthogonal validation of the proposed dataset suite are encouraged to implement specular reflections as part of their lighting analysis tools. Many daylight systems make use of specular elements, and it is expected that designers will wish to simulate them as they cannot be accounted for in rule of thumb calculations. 

Autodesk. (2010). Lesson 5: Optimizing the Design of the Building Envelope for Sustainability. In Autodesk Sustainable Design Curriculum 2010. Autodesk.

Autodesk. (2011, October 14). Global Illumination with the Mental Ray Renderer. Retrieved January 2012, from Autodesk 3ds Max Reference:

http://download.autodesk.com/us/3dsmax/2012help/index.html?url=files/GUID-B7D0424E-6DCB44D9-AD0B-85B9A1EE3F5-0.htm,topicNumber=d28e3788

Autodesk. (2011, October 14). Mental Ray Renderer. Retrieved January 2012, from Autodesk 3ds Max Reference: http://download.autodesk.com/us/3dsmax/2012help/index.html?url=files/GUIDB7D0424E-6DCB-44D9-AD0B-85B9A1EE3F5-0.htm,topicNumber=d28e3788

Chaos Software Ltd. (2007, November). GI Methods. Retrieved July 2012, from Chaos Group - Help Portal: http://www.spot3d.com/vray/help/150SP1/gimethods.htm

CIBSE. (1988). Technical Memoranda 14: CIBSE standard file format for the electronic transfer of luminaire photometric data. London: The Chartered Institution of Building Services Engineers.

CIE TC 3.33. (2005). Test cases to assess the accuracy of lighting computer programs. Vienne: Commission Internationale de L'Eclairage.

Dau Design and Consulting Inc. (2007). Validation of AGi32 against CIE 171:2006. Calgary: Dau Design and Consulting Inc.

DesignBuilder Software Ltd. (2011). Daylighting Calculation Options. Retrieved January 2012, from Design Builder Help:

http://www.designbuilder.co.uk/helpv3/Content/Daylighting\%20Calculation\%200ptions.htm

Donn, M., Xu, D., Harrison, D., \& Maamari, F. (2007). Using simulation software calibration tests as a consumer guide - a feasibility study using lighting simulation software. Building Simulation 2007 (pp. 1999-2006). Beijing: International Building Performance Simulation Association.

Dumortier, D., \& Kobav, M. (2007). Deriving CIE sky types from horizontal irradiances. Proceedings of the 26th Session of the CIE. Beijing: Commission Internationale de L'Eclairage.

Eppley Labs. (2004, May 25). Normal Incidence Pyrheliometer. Retrieved January 2012, from The Eppley Laboratory Inc.: http://www.eppleylab.com/PrdNormIncPyrhelmtr.htm

Galasiu, A. D., \& Reinhart, C. F. (2008). Current Daylighting Design Practice: A Survey. Building Research \& Information, 32(2), 159-174.

Geisler-Moroder, D., \& Dür, A. (2008, October 30-31). Validation of Radiance against CIE171:2006 and Improved Adaptive Subdivision of Circular Light Sources. Fribourg, Switzerland.

Grynberg, A. (1989). Validation of Radiance. Lighting Systems Research Group, LBL Technical Information Department. Berkeley, California: Lawrence Berkeley National Laboratory.

Hari, A. (2001). Integrated, Customer Driven, Conceptual Design Methodology, Doctoral Thesis. Haifa: Israel Institute of Technology.

Hopkinson, R. G., Petherbridge, P., \& Longmore, J. (1966). Daylighting. London, England: Heinemann.

IESNA. (2002). Standard File Format for Electronic Transfer of Photometric Data and Related Information. New York: Illuminating Engineering Society.

Ineichen, P., Molineaux, B., \& Perez, R. (1994). Sky luminance data validation: Comparison of seven models with four data banks. Solar Energy, 52(4), 337-346.

International Energy Agency. (2008). Annex 43 Testing and Validation of Building Energy Simulation Tools. Retrieved January 2012, from IEA Energy Conservation in Buildings and Community System: http://www.ecbcs.org/annexes/annex43.htm 
Jacobs, A. (2008, October 25). Basic Radiance Tutorial. Retrieved July 2009, from Learnix Documentation: http://luminance.londonmet.ac.uk/learnix/docs/radiance_tutorial.pdf

Jacobs, A. (2008, May 27). Radiance Cookbook. Retrieved July 2009, from Learnix - Documentation: http://luminance.Iondonmet.ac.uk/learnix/docs/radiance_cookbook.pdf

Jonsson, J. (2009, June 1). Generating layer BSDF using simulation and experiment. Retrieved December 2010, from Lawrence Berkeley National Laboratory: http://windows.lbl.gov/software/cfsworkshop/Presentation/Day1Generating\%20Layer\%20BSDF\%20using\%20Simulation\%20and\%20Experiment\%20\%20JCJonsson2003.pdf

Khodulev, A. B., \& Kopylov, E. A. (1996). Physically Accurate Lighting Simulation in Computer Graphics Software. International Conference on Computer Graphics and Visualization (GraphiCon '96). Moscow.

Kleindienst, S., Bodart, M., \& Andersen, M. (2008, July). Graphical Representation of Climate-Based Daylight Performance to Support Architectural Design. LEUKOS, 5(1), 39-61.

Konstantoglou, M. (2009, June 2). Visualizing Daylight Impacts. Retrieved December 2010, from Lawrence Berkeley National Laboratory: http://windows.lbl.gov/software/cfsworkshop/Presentation/Day2RadianceWorkshopPresentation.pdf

Konstantoglou, M., Jonsson, J. C., \& Lee, E. (2009). Simulating Complex Window Systems using BSDF Data. Plea2009 - 26th Conference on Passive and Low Energy Architecture. Quebec City. Retrieved from http://www.plea2009.arc.ulaval.ca/Papers/2.STRATEGIES/2.1\%20Daylighting/POSTER/2-1-21PLEA2009Quebec.pdf

Krygiel, E. (2010, December). Daylighting Analysis with Autodesk $\AA$ Revit $\circledast$ and Autodesk $3 d s$ Max $\circledast$ Design. Retrieved January 2012, from Autodesk University: http://au.autodesk.com/?nd=event_class\&session_id=7195\&jid=614811

LBNL. (2011, June 8). International Glazing Database. Retrieved July 2012, from Windows \& Daylighting: http://windows.lbl.gov/materials/IGDB/

Li, D., Lau, C., \& Lam, J. (2003). A Study of 15 Sky Luminance Patterns against Hong Kong Data. Architectural Science Review, 46(1), 61-68.

Li, D., Lau, C., \& Lam, J. (2004). Predicting Daylight Illuminance by Computer Simulation Techniques. Lighting Research Technology, 36(2), 113-129.

Maamari, F., Andersen, M., de Boer, J., Carroll, W. L., Dumortier, D., \& Greenup, P. (2006, July). Experimental validation of simulation methods for bi-directional transmission properties at the daylighting performance level. Energy and Buildings, 38(7), 878-889.

Mardaljevic, J. (1995). Validation of a Lighting Simulation Program Under Real Sky Conditions. Lighting Research \& Technology, 27(4), 181-188.

Mardaljevic, J. (1997). Validation of a Lighting Simulation Program: A study using Measured Sky Brightness Distributions. Lux Europa 97 Conference, (pp. 555-569). Amsterdam.

Mardaljevic, J. (2000). Simulation of annual daylighting profiles for internal illuminance. Lighting Research \& Technology, 32(3), 111-118.

Mardaljevic, J. (2000). Sky Models for Lighting Simulation. In J. Mardaljevic, Daylight Simulation: Validation, Sky Models and Daylight Coefficients (pp. 163-209). Leicester, United Kingdom: PhD Thesis, De Montfort University.

Mardaljevic, J. (2001). The BRE-IDMP dataset: a new benchmark for the validation of illuminance prediction techniques. Lighting Research \& Technology, 33(2), 117-134. 
Mardaljevic, J. (2002, October 10). A 'Gold Standard' dataset for the validation of illuminance predictions. Retrieved December 2010, from National Research Council Canada: http://www.nrccnrc.gc.ca/obj/irc/doc/gold-standard-dataset.pdf

Mardaljevic, J. (2003). Daylight Simulation. In G. Ward Larson, \& R. Shakespeare, Rendering with Radiance (Revised Edition ed., pp. 341-390). Davis, California, USA: Space and Light.

Mardaljevic, J. (2004). Verification of program accuracy for illuminance modelling: assumptions, methodology and an examination of conflicting findings. Lighting Research \& Technology, 36(3), 217-239.

Mardaljevic, J., \& Nabil, A. (2006). Useful daylight illuminances: A replacement for daylight factors. Energy and Buildings, 38(7), 905-913.

McNeil, A., \& Lee, E. (2012). A validation of the Radiance three-phase simulation method for modelling annual daylight performance of optically complex fenestration systems. Journal of Building Performance Simulation, 1-14.

Ochoa, C. E., Aries, M. B., \& Hensen, J. L. (2011, April 14). State of the Art in Lighting Simulation for Building Science: A Literature Review. Journal of Building Performance Simulation, 1-25.

Osborne, J. (2009). Shifting Forward: Validating Autodesk 3ds Max Design 2009's lighting analysis tools for daylight simulation in buildings. Wellington: Submitted for BBSc 401 - Research Methods; a requirement for the Bachelor of Building Science with Honours degree at Victoria University of Wellington.

Patton, J. (2007, October 20). 3ds Max 2008 Portal light tip. Retrieved November 2011, from mr Materials: http://www.mrmaterials.com/jeffs-blog/72-3ds-max-2008-portal-light-tip.html

Perez, R., Seals, R., \& Michalsky, J. (1993). All-Weather Model for Sky Luminance Distribution Preliminary Configuration and Validation. Solar Energy, 50(3), 235-245.

Pohlen, S., Ruck, B., \& Bittar, A. (1996). Evaluation of the Perez Luminous Efficacy Models for a Southern Hemisphere Site (New Zealand - $\left.41^{\circ} \mathrm{S}, 175^{\circ} \mathrm{E}\right)$. Solar Energy, 57(4), 307-315.

Reinhart, C. F. (2005). A simulation-based review of the ubiquitous window-head-height to daylit zone depth rule of thumb. Building Simulation 2005. Montreal: IBPSA.

Reinhart, C. F. (2009). Experimental Validation of Autodesk 3ds Max Design 2009 and Daysim 3.0. Autodesk Canada Co.

Reinhart, C. F., \& Andersen, M. (2006, July). Development and validation of a Radiance model for a translucent panel. Energy and Buildings, 38(7), 890-904.

Reinhart, C. F., \& Fitz, A. (2006). Findings from a Survey on the Current Use of Daylight Simulations in Building Design. Energy And Buildings, 38, 824-835.

Reinhart, C. F., \& Herkel, S. (2000). The Simulation of Annual Daylight Illuminance Distributions - a StateOf-The-Art Comparison of Six RADIANCE-Based Methods. Energy And Buildings, 32, 167-187.

Reinhart, C. F., \& Walkenhorst, O. (2001). Validation of Dynamic RADIANCE-Based Daylight Simulations for a Test Office With External Blinds. Energy And Buildings, 33, 683-697.

Reinhart, C. F., \& Weissman, D. A. (2012). The daylit area - Correlating architectural student assessments with current and emerging daylight availability metrics. Building and Environment, 50, 155-164.

Ruck, N., Aschehoug, Ø., Aydinli, S., Christoffersen, J., Courret, G., Edmonds, I., . . Selkowitz, S. (2000). Daylighting Systems. In N. Ruck, $\varnothing$. Aschehoug, S. Aydinli, J. Christoffersen, G. Courret, I. Edmonds, ... S. Selkowitz, Daylight in Buildings - A Source Book on Daylighting Systems and Components (pp. 4.1-4.104). Berkeley, California, USA: Lawrence Berkeley National Laboratory.

Shahriar, A., \& Mohit, M. (2006). Frequency Distribution of CIE Standard General Skies for Subang, Malaysia. Architectural Science Review, 49(4), 363-366. 
Shlick, C. A. (1993). A Customizable Reflectance Model for Everyday Rendering. Fourth Eurographics Workshop on Rendering (pp. 73-84). Paris: Eurographics.

Turner, D. P. (1969). Windows and environment. Newton-le-Willows, United Kingdom: McCorquodale.

Ward, G. (1994). Setting Rendering Options. Retrieved January 2012, from Radiance Synthetic Imaging System Reference: http://radsite.lbl.gov/radiance/refer/Notes/rpict_options.html

Ward, G. (2004, September 21). Behavior of Materials in RADIANCE. Retrieved March 2012, from Radiance Synthetic Imaging System: http://radsite.lbl.gov/radiance/refer/materials.pdf

Ward, G., \& Shakespeare, R. (2003). Rendering with Radiance (Revised ed.). Davis, California, USA: Space and Light.

Ward, G., Mistrick, R., Lee, E., McNeil, A., \& Jonsson, J. (2011). Simulating the Daylight Performance of Complex Fenestration Systems Using Bidirectional Scattering Distribution Functions within Radiance. LEUKOS, 7(4). Retrieved from http://gaia.lbl.gov/btech/papers/4414.pdf

X-Rite Incorporated. (2008). ColorMunki Design. Retrieved February 2012, from ColorMunki: http://www.colormunki.com/product/show?is_designer_type=true 


\subsection{Appendix 1: 3ds Max Design \& Radiance simulation}

\section{options \& parameters}

This section lists the simulation parameters used for 3ds Max Design and Radiance for the majority of the simulations undertaken in this study. Any changes to these parameters are described in their relevant sections.

Table 9: 3ds Max Design / Mental Ray simulation parameters

\begin{tabular}{|c|c|c|}
\hline 3ds Max Render Dialog Rollout & Section & Parameter \\
\hline \multirow[t]{5}{*}{ Rendering Algorithms } & Scanline & Enable: Off \\
\hline & \multirow[t]{4}{*}{ Raytracing } & Enable: On \\
\hline & & Max Trace Depth: 10 \\
\hline & & Max Trace Reflections: 10 \\
\hline & & Max Trace Refractions: 10 \\
\hline \multirow[t]{2}{*}{ Shadows \& Displacement } & \multirow[t]{2}{*}{ Shadows } & Enable: On \\
\hline & & Mode: Simple \\
\hline \multirow[t]{13}{*}{ Final Gather } & \multirow[t]{7}{*}{ Basic } & Enable Final Gather: On \\
\hline & & Multiplier: 1.0 \\
\hline & & Initial Final Gather Point Density: 1.0 \\
\hline & & Rays per FG Point: 2500 \\
\hline & & Interpolate Over Num. FG Points: 5 \\
\hline & & Diffuse Bounces: 6 \\
\hline & & Weight: 1 \\
\hline & \multirow[t]{5}{*}{ Advanced } & Noise Filtering: None \\
\hline & & Max Depth: 10 \\
\hline & & Max Reflections: 10 \\
\hline & & Max Refractions: 10 \\
\hline & & Use Falloff (Limit Ray Distance): Off \\
\hline & FG Point Interpolation & Use Radius Interpolation Method: Off \\
\hline \multirow[t]{2}{*}{ Caustics \& Global Illumination (GI) } & Caustics & Enable: Off \\
\hline & Global Illumination & Enable: Off \\
\hline
\end{tabular}


Table 10: Radiance rtrace simulation parameters

\begin{tabular}{|c|c|c|}
\hline Option & Value & Description \\
\hline-1 & $\mathrm{~N} / \mathrm{A}$ & $\begin{array}{l}\text { Compute irradiance rather than radiance, with the input as measurement } \\
\text { point and orientation. }\end{array}$ \\
\hline$-\mathrm{h}$ & $\mathrm{N} / \mathrm{A}$ & Include information header on output. \\
\hline$-d p$ & 2046 & $\begin{array}{l}\text { Sets the secondary source presampling density to } 2046 \text { samples per } \\
\text { steradian. For } 2 \pi \text { steradians in a hemisphere this is roughly the same as } \\
2500 \times 5 \text { Final Gather rays. }\end{array}$ \\
\hline -ar & Varies & This value is set for each simulation by equation 3 below. \\
\hline- ds & 0.15 & $\begin{array}{l}\text { Sets the direct sampling value to } 0.15 \text {. Differs from Reinhart's value of } 0 \\
\text { as this study includes area light sources in some datasets. }\end{array}$ \\
\hline$-\mathrm{dt}$ & 0 & $\begin{array}{l}\text { Sets the Direct Threshold value to } 0 \text {; meaning all light sources in a scene } \\
\text { are tested for shadows. }\end{array}$ \\
\hline$-d c$ & $\mathrm{~N} / \mathrm{A}$ & $\begin{array}{l}\text { Normally sets the Direct Certainty value, but is not required with the Direct } \\
\text { Threshold (-dt) value set to } 0 \text {. }\end{array}$ \\
\hline$-d r$ & 3 & $\begin{array}{l}\text { Sets the number of relays for secondary sources to 3; causing first, } \\
\text { second and third generation secondary sources to all be made into third } \\
\text { generation secondary sources. }\end{array}$ \\
\hline$-s j$ & 1 & $\begin{array}{l}\text { Sets the specular sampling jitter to 1; ensuring all highlights are fully } \\
\text { sampled using raytracing. }\end{array}$ \\
\hline -st & 0.1 & $\begin{array}{l}\text { Sets the specular sampling threshold to } 0.1 \text {, meaning most specular } \\
\text { reflections are fully traced, rather than approximated. }\end{array}$ \\
\hline$-a b$ & 6 & Sets the maximum number of diffuse bounces to 6 . \\
\hline -aa & 0.05 & $\begin{array}{l}\text { Sets the ambient accuracy to } 0.05 \text {; allowing for a small amount of } \\
\text { illuminance interpolation. }\end{array}$ \\
\hline -ad & 2500 & $\begin{array}{l}\text { Sets the number of Ambient Divisions to } 2500 \text {, the same as } 2500 \text { Final } \\
\text { Gather rays. This differs from Reinhart's value of } 1500 \text {. }\end{array}$ \\
\hline -as & 100 & $\begin{array}{l}\text { Sets the number of super-samples (extra samples between two ambient } \\
\text { divisions with a significant difference) to } 100 \text {. }\end{array}$ \\
\hline -av & 0.000 .000 .00 & $\begin{array}{l}\text { Sets the RGB radiance ambient values to } 0.00 \text { recommended for daylight } \\
\text { simulations (Mardaljevic, Daylight Simulation, 2003). }\end{array}$ \\
\hline$-\operatorname{lr}$ & 10 & Limits reflections to a maximum of 10 . \\
\hline$-\mathrm{Iw}$ & 0.0005 & $\begin{array}{l}\text { Limits the weight of each ray to a minimum of } 0.0005 \text {. Ensures Russian } \\
\text { roulette is not used to determine whether a ray is traced. }\end{array}$ \\
\hline
\end{tabular}

Equation 3: Equation for setting -ar parameter in Radiance (Mardaljevic, 2003)

$$
S_{\min }=\frac{D_{\max } \times-a a}{-a r}
$$

Where $S_{\min }$ is the minimum separation for cached irradiances, $D_{\max }$ is the maximum scene dimension, and -aa is the ambient accuracy value listed in the table above. 
This section contains additional error sparklines for the LBNL 71T test-bed's walls and ceiling and a breakdown of each of the individual day's calculated MBEs. These relate to the results described in chapter 5.

Figure 54: LBNL 71T test-bed facility ceiling \& wall sparklines - 3ds Max Design

\begin{tabular}{|c|c|}
\hline \multicolumn{2}{|c|}{ Ceiling Sensor } \\
\hline $0.0 \%, 0.0 \%, 0.0 \%$ & $1.3 \%, 0.3 \%, 0.0 \%$ \\
\hline $62.1 \%, 33.0 \%, 12.4 \%$ & $11.0 \%, 0.2 \%, 0.0 \%$ \\
\hline $65.9 \%, 32.0 \%, 10.9 \%$ & $13.7 \%, 0.0 \%, 0.0 \%$ \\
\hline $0.5 \%, 0.0 \%, 0.0 \%$ & $63.5 \%, 4.9 \%, 1.6 \%$ \\
\hline $7.8 \%, 0.8 \%, 0.5 \%$ & $57.5 \%, 15.5 \%, 7.1 \%$ \\
\hline $6.6 \%, 3.8 \%, 2.0 \%$ & $3.3 \%, 0.2 \%, 0.2 \%$ \\
\hline
\end{tabular}

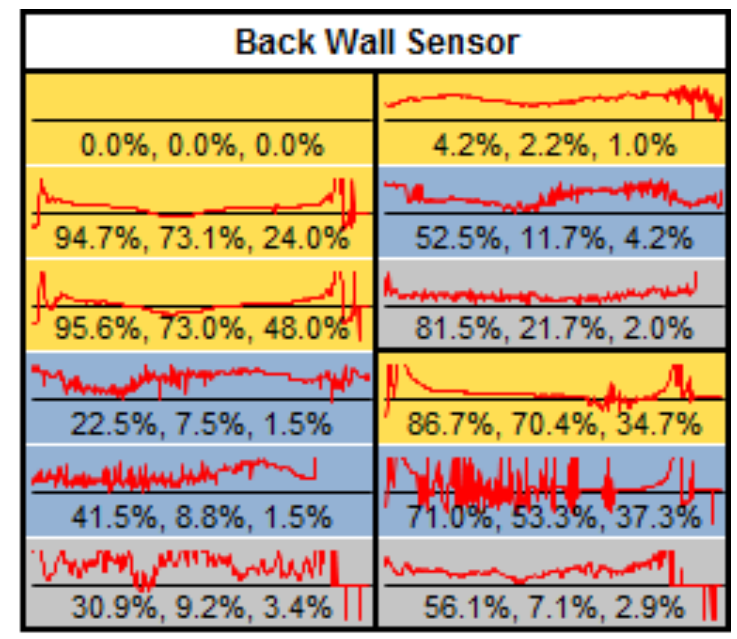

\begin{tabular}{|c|c|}
\hline \multicolumn{2}{|c|}{ West Wall Sensor } \\
\hline 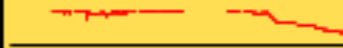 & 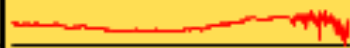 \\
\hline $6.2 \%, 0.0 \%, 0.0 \%$ & $17.0 \%, 1.2 \%, 0.3 \%$ \\
\hline$\mu$ & 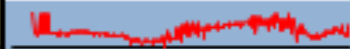 \\
\hline $94.1 \%, 66.9 \%, 47.4 \%$ & $49.5 \%, 11.3 \%, 2.3 \%$ \\
\hline & ل لس \\
\hline $94.7 \%, 65.3 \%, 49.5 \%$ & $73.5 \%, 10.7 \%, 0.2 \%$ \\
\hline 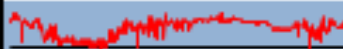 & \\
\hline $24.2 \%, 15.8 \%, 6.8 \%$ & $92.4 \%, 62.0 \%, 11.4 \%$ \\
\hline 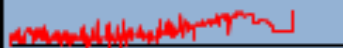 & $\mathbb{B} \mid \|_{11}, d$ \\
\hline $51.2 \%, 22.5 \%, 6.7 \%$ & $72.4 \%, 49.2 \%, 23.1 \%$ \\
\hline 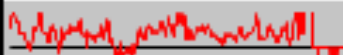 & بيني \\
\hline $56.7 \%, 17.4 \%, 6.0 \%$ & $91.8 \%, 62.0 \%, 30.2 \%$ \\
\hline
\end{tabular}

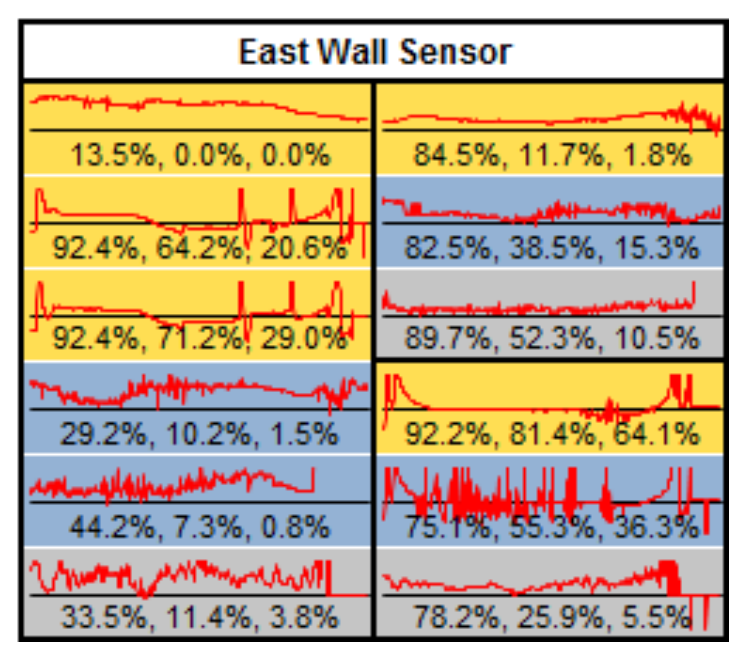


Figure 55: LBNL 71T test-bed facility ceiling \& wall sparklines - Radiance

\begin{tabular}{|c|c|}
\hline \multicolumn{2}{|c|}{ Ceiling Sensor } \\
\hline L & mines. \\
\hline $0.0 \%, 0.0 \%, 0.0 \%$ & $42.0 \%, 3.5 \%, 0.7 \%$ \\
\hline$a^{2}=$ & - \\
\hline $83.8 \%, 62.9 \%, 27.4 \%$ & $52.2 \%, 9.7 \%, 1.0 \%$ \\
\hline 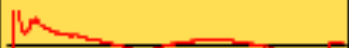 & 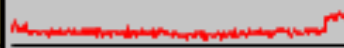 \\
\hline $83.8 \%, \overline{62} .1 \%, 33.5 \%$ & $77.2 \%, 2.0 \%, 0.2 \%$ \\
\hline 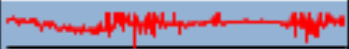 & 以 \\
\hline $3.5 \%, 0.3 \%, 0.2 \%$ & $78.6 \%, 39.4 \%, 7.5 \%$ \\
\hline 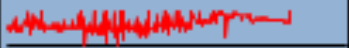 & $\Delta\left\|_{1}\right\|^{\|}$. \\
\hline $7.8 \%, 0.8 \%, 0.5 \%$ & $62.4 \%, 23.5 \%, 6.9 \%$ \\
\hline 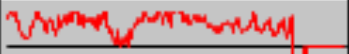 & \\
\hline $14.6 \%, 5.2 \%, 2.8 \%$ & $91.8 \%, 44.3 \%, 11.0 \%$ \\
\hline
\end{tabular}

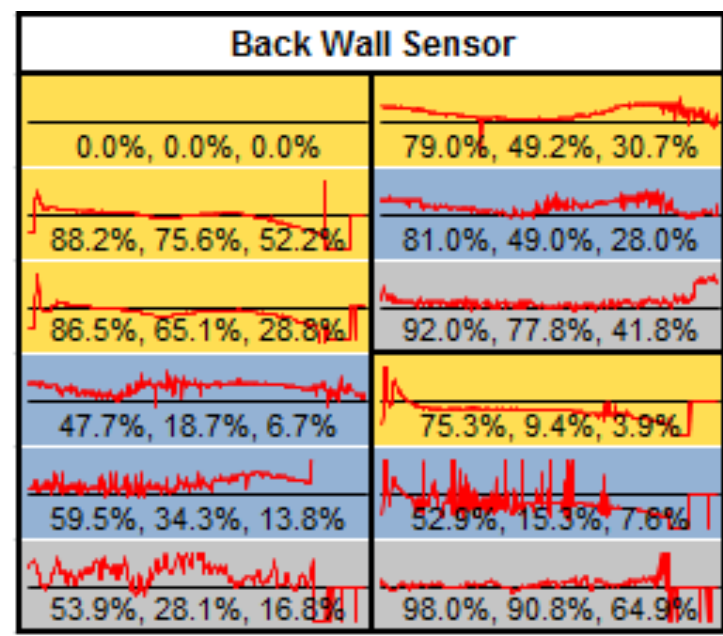

\begin{tabular}{|c|c|}
\hline \multicolumn{2}{|c|}{ West Wall Sensor } \\
\hline 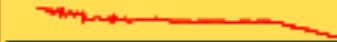 & \\
\hline $14.5 \%, 5.7 \%, 0.7 \%$ & $85.3 \%, 60.0 \%, 44.7 \%$ \\
\hline ل & $=m$ \\
\hline $64.8 \%, 29.0 \%, 23.0 \%$ & $84.2 \%, 64.7 \%, 37.7 \%$ \\
\hline H. & \\
\hline $651 / \%,-97.3 \%, 24.6 \%$ & $91.5 \%, 88.2 \%, 56.0 \%$ \\
\hline 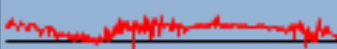 & . \\
\hline $63.2 \%, 25.0 \%, 14.2 \%$ & $64.3 \%, 6.7 \%, 2.5 \%$ \\
\hline 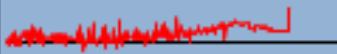 & \\
\hline $69.0 \%, 42.7 \%, 26.7 \%$ & $19.8 \%, 4.7 \%$ \\
\hline 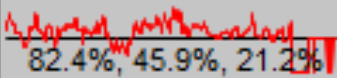 & $\overline{98.4 \%}, \overline{9} 2.0 \%, 61.0 \%$ \\
\hline
\end{tabular}

\begin{tabular}{|c|c|}
\hline \multicolumn{2}{|c|}{ East Wall Sensor } \\
\hline 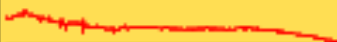 & \\
\hline $30.7 \%, 10.2 \%, 5.8 \%$ & $99.5 \%, 70.7 \%, 54.2 \%$ \\
\hline & \\
\hline $76.8 \%, 66.3 \%, 48.8 \%$ & $99.5 \%, 76.7 \%, 47.7 \%$ \\
\hline & \\
\hline $77.7 \%, 67.2 \%, 48.4 \%$ & $93.0 \%, 90.2 \%, 75.2 \%$ \\
\hline لئبس & \\
\hline $89.0 \%, 30.2 \%, 13.5 \%$ & $68.6 \%, 7.3 \%, 2.2 \%$ \\
\hline لـ & \\
\hline $69.2 \%, 39.3 \%, 24.3 \%$ & $50.6 \%, 14.1 \%, 5.5 \%$ \\
\hline 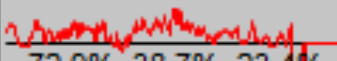 & \\
\hline $72.9 \%, 38.7 \%, 23.4 \%$ & $97.6 \%, 94.9 \%, 73.9 \%$ \\
\hline
\end{tabular}

Table 11: LBNL 71T daily workplane Mean Bias Errors \& data points

\begin{tabular}{|l|l|l|l|l|l|l|}
\hline Window Condition: & Unshaded \\
\hline Date/Weather & $17-J u n-10$ & $15-J a n-11$ & $16-J a n-11$ & $18-J u n-10$ & $19-J u n-10$ & $4-D e c-10$ \\
\hline 3ds Max Design MBE & $65.25 \%$ & $8.31 \%$ & $-0.40 \%$ & $37.46 \%$ & $26.54 \%$ & $17.67 \%$ \\
\hline Radiance MBE & $41.10 \%$ & $-13.87 \%$ & $-70.09 \%$ & $18.95 \%$ & $11.88 \%$ & $5.84 \%$ \\
\hline \# of data points & 3600 & 1794 & 1794 & 3600 & 3026 & 3006 \\
\hline Window Condition: & Tilt 0 & \multicolumn{5}{|l|}{ Tilt 45 } \\
\hline Date/Weather & $19-J u l-10$ & $20-J u l-10$ & $21-J u l-10$ & $30-$ Dec-10 & 29-Dec-10 & $28-$ Dec-10 \\
\hline 3ds Max Design MBE & $26.56 \%$ & $18.24 \%$ & $13.48 \%$ & $9.84 \%$ & $7.79 \%$ & $24.09 \%$ \\
\hline Radiance MBE & $-1.76 \%$ & $-3.05 \%$ & $-6.93 \%$ & $-32.24 \%$ & $-32.89 \%$ & $-5.86 \%$ \\
\hline \# of data points & 3600 & 3600 & 3600 & 3060 & 3060 & 3060 \\
\hline
\end{tabular}

